Portland State University

PDXScholar

$1-7-2021$

\title{
Development of Graphene Synthesis and Characterization Techniques Toward CMOS Applications and Beyond
}

Otto Kno Zietz

Portland State University

Follow this and additional works at: https://pdxscholar.library.pdx.edu/open_access_etds

Part of the Materials Science and Engineering Commons Let us know how access to this document benefits you.

\section{Recommended Citation}

Zietz, Otto Kno, "Development of Graphene Synthesis and Characterization Techniques Toward CMOS Applications and Beyond" (2021). Dissertations and Theses. Paper 5662.

https://doi.org/10.15760/etd.7534

This Dissertation is brought to you for free and open access. It has been accepted for inclusion in Dissertations and Theses by an authorized administrator of PDXScholar. Please contact us if we can make this document more accessible: pdxscholar@pdx.edu. 
Development of Graphene Synthesis and Characterization Techniques toward CMOS

Applications and Beyond

by

Otto Kno Zietz

A dissertation submitted in partial fulfillment of the requirements for the degree of

\author{
Doctor of Philosophy \\ in \\ Mechanical Engineering \\ Dissertation Committee: \\ Jun Jiao, Chair \\ Tae-Kyu Lee \\ Zhiqiang Chen \\ Thomas Schumacher
}

Portland State University

2021 


\begin{abstract}
Graphene exhibits mechanical and electrical properties which, coupled with its two dimensional (2D) morphology, make it an attractive material component for inclusion in a wide range of industries. Since the discovery of graphene in 2004, industry adoption has been limited due to the demanding synthesis requirements for high quality and connected graphene as well as the difficulties associated with direct incorporation. Chemical vapor deposition (CVD) has emerged as the most cost efficient method for producing high quality graphene at scales suitable for mass production. However, the $1000^{\circ} \mathrm{C}$ temperatures and micrometer thick catalysts required for this process preclude direct inclusion in applications with topographically varied surfaces as graphene is produced in planar sheets that must be transferred.
\end{abstract}

One attractive application for graphene is as a diffusion barrier in CMOS applications as the single atom thick material has shown significant ability to block copper diffusion at elevated temperatures. For realization of this application, both the required catalyst thicknesses and synthesis temperatures for graphene production must be reduced to enable direct graphene incorporation on these nanoscale and nonplanar surfaces without thermal damage to existing components. A second application in which graphene inclusion would be beneficial is the field of spintronics, in which the spin orientation of electrons are used as an additional degree of freedom for computation and information storage. This beyond-CMOS application represents an avenue for significant improvement over current technologies and graphene, with its weak spin orbit coupling and high electron mobility, displays potential as a long-distance spin transport component of future spintronic devices. 
Characterization of graphene's spin transport properties has been primarily investigated in a nonlocal spin valve device (NLSV), resulting in experimental spin transport parameters orders of magnitude below those theoretical predicted. To advance graphene as a component for future spintronic applications, new device designs to explore spin transport phenomena not detectable in NLSV devices as well as scalable fabrication techniques will be needed.

In this work, we develop graphene synthesis techniques to reduce required temperatures through hydrocarbon precursor control during plasma enhanced chemical vapor deposition (PECVD). Through manipulation of the size and ionization state of hydrocarbon precursors that interact with the growth catalyst, we demonstrate $95 \%$ fewto-monolayer graphene synthesis at $500^{\circ} \mathrm{C}$ on $50 \mathrm{~nm}$ catalysts, representing a 10 -fold reduction in catalyst thickness requirements at temperatures approaching the limit for direct incorporation in CMOS applications. Additionally, we demonstrate manipulation of metal catalyst morphology and composition toward controlling graphene layer number, defect types, and uniformity. Characterization of trimetallic catalysts, compared to single metal or bimetallic catalysts traditionally examined in literature, reveal that low temperature graphene synthesis pathways can be manipulated through small additions of less reactive metals (Gold and Copper) to primarily high reactivity metal catalysts (Ni) through both energetic and surface modulation resulting in monolayer graphene synthesis.

While low temperature graphene synthesis techniques are needed for graphene incorporation in current CMOS products, beyond-CMOS applications do not necessarily require temperature restrictions on synthesis as fabrication of these devices can implement 
planar graphene as the first device component. To characterize graphene as a spin transport channel, commercially available graphene grown at elevated temperatures is used to address spin transport properties through design of a novel device configuration, the hybrid drift diffusion spin valve (HDDSV), in which an additional transport channel is added to the standard NLSV. This device architecture has not been previously studied and is aimed at revealing magnetic contact effects on graphene spin transport as well as exploring drift and diffusion interactions with respect to achievable spin signals. Wafer scale fabrication of these devices is demonstrated and processing techniques are optimized to enable spin signal detection on arrays containing 120 individual devices. Characterization of the new HDDSV configuration reveals changes to detected spin signals in both the standard NLSV portion and the added channel, revealing spin signals as large as $865 \Omega$ in the additional transport channel compared to an average signal of $7.3 \Omega$ in the traditional configuration. The additional channels also exhibit detectable spin signal under a 3 point local measurement, representing a potential avenue toward long distance spin transport and enabling increased device complexity that will be necessary for the realization of graphene based spintronic devices.

These findings represent the development of graphene synthesis and characterization techniques aimed at advancing fundamental understanding and enabling further practical application. The methods developed in this study serve as new avenues for continued improvement toward direct incorporation of a material that has the potential to revolutionize a number of fields. 


\section{Table of Contents}

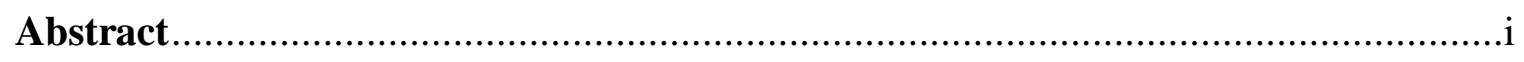

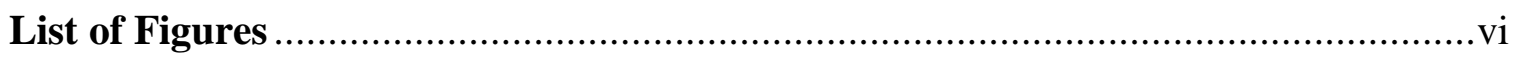

List of Abbreviations .......................................................................................

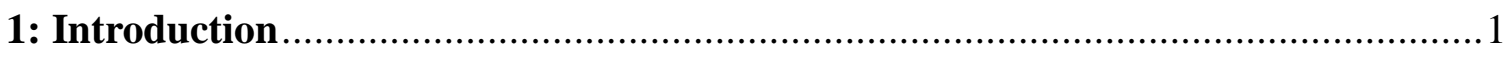

1.1: Graphene Overview: Properties, Applications, and Synthesis Techniques .....2

1.2: Graphene Synthesis via Chemical Vapor Deposition ................................. 3

1.2.1: The Chemical Vapor Deposition Mechanism .............................5

1.2.2: Graphene Quality Determination via Raman Spectroscopy ...........6

1.2.3: Challenges Associated with Low Temperature Graphene

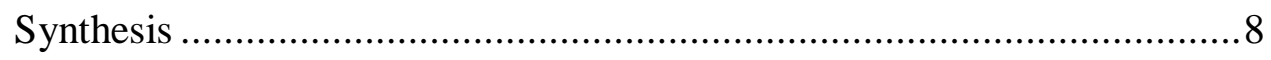

1.3: Graphene as a Spin Transport Channel ................................................... 11

1.3.1: Investigation of Graphene's Spin Transport Properties .................13

1.3.2: Challenges Associated with Measuring and Manipulating Graphene

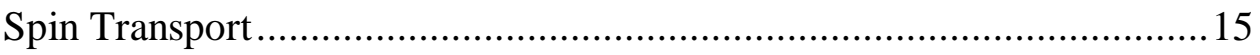

1.3.3: The Role of FM Contacts on Spin transport in a Standard Graphene

NLSV

1.3.4: Hybrid Effect of Spin Drift and Diffusion in a Graphene NLSV ....18

1.4: Problem Statement and Approach........................................................20

2: Graphene Synthesis via Plasma Enhanced Chemical Vapor Deposition .............23

2.1: Hydrocarbon Precursor Manipulation .................................................23

2.1.1 Introduction .................................................................24

2.1.2 Results and Discussion....................................................25

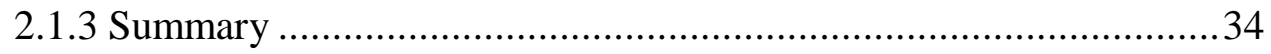

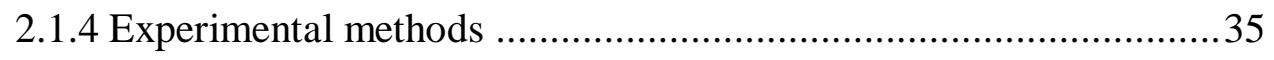

2.2: Catalyst Design and Characterization ................................................. 37

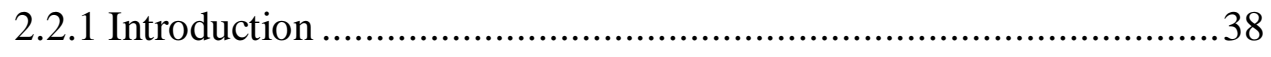


2.2.2 Results and Discussion.

2.2.3 Summary

2.2.4 Experimental methods .50

2.3 From CMOS to beyond-CMOS applications .........................................51

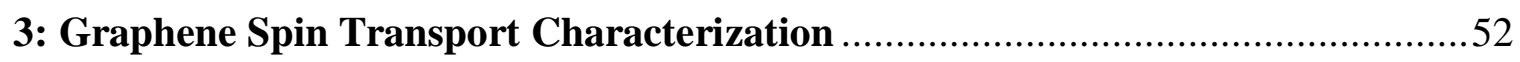

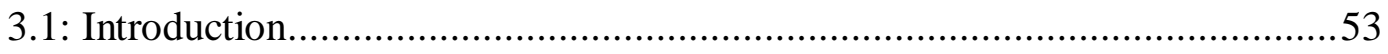

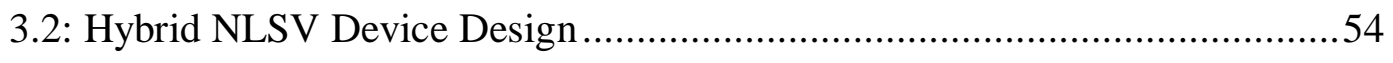

3.3: Wafer Scale Device Fabrication .......................................................59

3.4: HDDSV Device Characterization .....................................................69

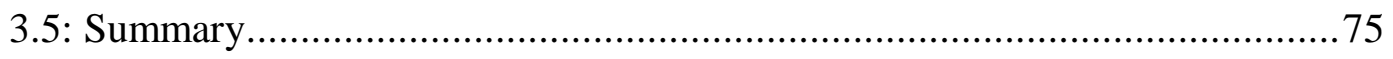

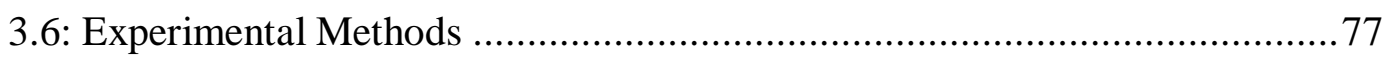

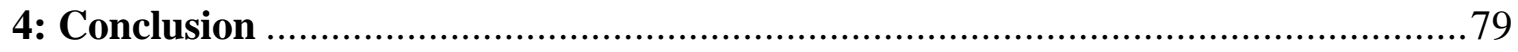

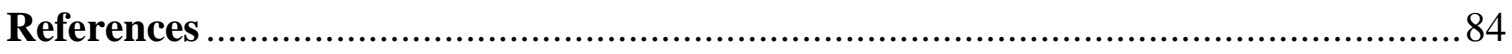




\section{List of Figures}

Figure 1.1: Comparison of CVD and PECVD techniques for graphene synthesis ...........5

Figure 1.2: Typical Raman spectra detected from graphene ...................................

Figure 1.3: Standard non-local spin valve device .............................................. 15

Figure 2.1: Schematic of custom PECVD system and characterization capabilities .......25

Figure 2.2: Characterization of PECVD reaction environment ...............................2 27

Figure 2.3: Comparison of graphene synthesis as a function of screening bias..............29

Figure 2.4: Comparison of multistage graphene synthesis regimes .............................31

Figure 2.5: Graphene synthesis with both ICP and secondary CCP ...........................33

Figure 2.6: Comparison of graphene synthesized on various metal catalysts ................40

Figure 2.7: EBSD maps from growth catalysts with varied $\mathrm{Au}$ and $\mathrm{Cu}$ composition......42

Figure 2.8: Simulation results for acetylene decomposition...................................45

Figure 2.9: Electrochemical transfer technique for catalyst thin films .........................47

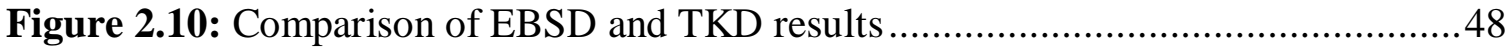

Figure 2.11: TEM images from transferred metal thin film catalysts ..........................49

Figure 3.1: Schematic of hybrid nonlocal spin valve device design ..........................54

Figure 3.2: Schematic of device configurations for spin transport characterization........57

Figure 3.3: 120 device array of graphene devices for wafer scale fabrication ...............59

Figure 3.4: Optical images of wafer scale device fabrication processing steps..............60

Figure 3.5: AFM images displaying forming gas anneal removal of residues ..............61

Figure 3.6: Representative spin signal from NLSV devices with varied TB oxidation treatments with a comparison of contact resistances under each condition ...................64

Figure 3.7: SEM images indicating variation in graphene device dimensions ................66

Figure 3.8: Comparison of NLSV control device parameters before and after ambient exposure

Figure 3.9: Magnetic sweep data displaying pre- and post-ambient exposure $\Delta R_{N L}$

signals

Figure 3.10: SEM images displaying the fabricated FM/TB and graphene channel orientations

Figure 3.11: Magnetic sweep data of HDDSV devices measured in the standard NLSV configuration with accompanying SEM image

Figure 3.12: Magnetic sweep data of HDDSV devices measured in the hybrid configuration with accompanying SEM image 


\section{List of Abbreviations}

CMOS - complementary metal oxide semiconductor

NLSV - nonlocal spin valve

HDDSV - hybrid drift diffusion spin valve

CVD - chemical vapor deposition

PECVD - plasma enhanced chemical vapor deposition

SOC - spin orbit coupling

FM - ferromagnetic

$\mathrm{NM}$ - nonmagnetic

SLG - single layer graphene

BLG - bilayer graphene

MLG - multilayer graphene

TB - tunnel barrier

PVD - physical vapor deposition

ICP - inductively coupled plasma

$\mathrm{CCP}$ - capacitively coupled plasma

MS - mass spectrometer

EBSD - electron backscatter diffraction

EDX - x-ray spectroscopy

TEM - transmission electron microscopy

SEM - scanning electron microscopy

TKD - transmission Kikuchi diffraction

IPF- inverse pole figure

$\mathrm{EBE}$ - electron beam evaporation

AFM - atomic force microscopy 


\section{1: Introduction}

The discovery of graphene in $2004^{1}$ marked the realization of a new class of materials, two dimensional (2D) atomic crystal systems, which had previously only been theoretically predicted. ${ }^{2}$ Graphene is a honeycomb lattice of $\mathrm{sp}^{2}$ bonded carbon atoms whose single atomic thickness, $0.14 \mathrm{~nm}$ bond length, and free electron in the out-of-plane $\pi$ orbital lead to extraordinary mechanical, optical, thermal, and electrical properties. In the 16 years since the initial isolation of graphene, intense research has occurred in both academic and industry settings to characterize graphene's unique properties, develop synthesis techniques suitable for high-quality and scalable production, and advance avenues for graphene incorporation in a wide variety of fields.

Despite intensive investigative efforts over the past decade, graphene adoption by industry has been slow, primarily due to the demanding synthesis requirements for high quality and connected graphene film production as well as the difficulties associated with graphene transfer to targeted non-planar substrates. These challenges have resulted in graphene inclusion in only a small number of commercial products through synthesis processes that utilize imperfect, multilayer, and disconnected graphene domains not exhibiting the achievable properties of pristine and connected graphene. One objective in this dissertation research is to identify and develop synthesis techniques to improve achievable graphene quality through processes amenable to semiconductor industry incorporation and develop new device architectures to characterize graphene spin transport properties toward the advancement of graphene as a material component for the next generation of nanoelectronics. 
1.1: Graphene Overview: Properties, Applications, and Synthesis Techniques

Graphene was first experimentally isolated via mechanical exfoliation from graphite in 2004 and launched intense research activities to characterize the zero band gap semiconductor's properties, in which electrons in the out of plane $\pi$-orbital behave as massless particles ${ }^{3}$, and develop scalable methods for production. Pristine graphene isolated through exfoliation is the strongest material yet discovered, with a Young's modulus of $1 \mathrm{TPa}$ and intrinsic strength of $130 \mathrm{GPa},{ }^{4}$ and, when suspended, exhibits ballistic transport over millimeter scales at room temperature ${ }^{5}$ with an achievable electron mobility of $2.5 \times 10^{5} \mathrm{~cm}^{2} \mathrm{~V}^{-1} \mathrm{~s}^{-1}$. Additionally, graphene exhibits anisotropic thermal conductivity, in-plane $300 \mathrm{~W} \mathrm{mK}{ }^{-1}$ and out-of-plane $7 \mathrm{mK}^{-1},{ }^{6} 10^{6}$ higher current densities than copper ${ }^{7}$ and 100 times the mobility of Silicon ${ }^{8}$. Graphene's sp ${ }^{2}$ bonded carbon atoms exhibit weak spin orbit coupling (SOC) $)^{9}$ enabling the spin state of injected electrons to travel long distances undisturbed. Finally, graphene's electronic properties can be influenced by the nearest neighbor material, termed the proximity effect, due to the single atomic thickness and surface interactions driven by the out of plane $\pi$-orbital network. ${ }^{10,11}$

With applications ranging from flexible electronics ${ }^{12}$, transistors $^{13}$, diffusion barriers ${ }^{14}$, energy generation and storage ${ }^{15}$, high strength composite materials ${ }^{16}$, and interconnects for spintronic applications and quantum computing ${ }^{17}$ the potential exists for graphene to revolutionize a wide variety of industries. However, the extraordinary properties exhibited by pristine graphene, free from grain boundaries, vacancies, and out of plane $\mathrm{sp}^{3}$ hybridization or doping of the film, are significantly diminished as defect concentrations increase. ${ }^{18-20}$ 
With sizes of mechanically exfoliated graphene limited to the centimeter scale, incorporation by applications that would benefit from large-area connected films with low defect densities require the development of scalable graphene synthesis procedures. Numerous graphene synthesis processes have been identified, including liquid phase and thermal exfoliation ${ }^{21}$, molecular assembly ${ }^{22}$, synthesis from silicon carbide ${ }^{23}$, and chemical vapor deposition (CVD) ${ }^{24}$, each with varying costs, achievable domain sizes and quality, as well as synthesis and catalyst requirements. Of these methods, CVD currently represents the best achievable quality through scalable and cost efficient processes that are currently used in semiconductor industry applications, though high synthesis temperatures, thick catalyst requirements, and transfer limitations remain as roadblocks to incorporation. ${ }^{25}$

\section{2: Graphene Synthesis via Chemical Vapor Deposition}

Chemical vapor deposition (CVD) has emerged as a promising route for scalable graphene synthesis enabling quality and layer number control suitable for CMOS applications requiring connected films with reduced defect densities. Graphene synthesis via CVD occurs through exposure of a target substrate to a carbon containing precursor followed by dehydrogenation of the carbon precursor resulting in active carbon species available for graphene formation. ${ }^{26}$ The temperature required for graphene synthesis, the number of graphene layers, and the quality of the resultant film are dictated by the catalytic activity and carbon solubility of target substrate, the stability and size of the carbon precursor, and the reaction environment conditions. First attempts were made with nickel $(\mathrm{Ni})$ and iron $(\mathrm{Fe})$ catalyst materials, resulting in inhomogeneous films dense in 
bilayer, few-layer, and many-layer regions. ${ }^{27}$ Later, rapid thermal annealing of $\mathrm{SiO}_{2}$ supported Ni films was introduced to produce monolayer graphene at $1080{ }^{\circ} \mathrm{C} .{ }^{28} \mathrm{Copper}$ as a catalyst for graphene growth was first introduced by Li and co-workers in 2009, who reported large-areas of high-quality and uniform graphene formed on $\mathrm{Cu}$ foil. ${ }^{29}$ They suggest that $\mathrm{Cu}$ exhibits self-limited growth characteristics, due to its relatively low carbon solubility in contrast to $\mathrm{Ni}, \mathrm{Fe}$ and most other transition metals. Recent advancements in CVD graphene using $\mathrm{Cu}$ catalysts have greatly refined this process and even identified parameters for growing few-layer graphene through variation of the $\mathrm{Cu}$ surface morphology. ${ }^{30}$

Other critical CVD parameters have also been investigated, such as hydrogen $\left(\mathrm{H}_{2}\right)$ concentration relative to hydrocarbon gas precursors as well as the use of liquid phase carbon precursors to reduce required synthesis temperatures. ${ }^{31-34}$ The vast majority of large-area graphene to date (including roll-to-roll graphene ${ }^{35}$ ) has been grown using thick $\mathrm{Cu}$ foils as both growth catalyst and substrate..$^{29,30,32,33,36}$ This graphene fabrication technique has enabled successful companies in the US and overseas to provide graphene to both industrial and academic customers. Though this has increased graphene availability, synthesis temperatures remain in excess of $800^{\circ} \mathrm{C}$ and catalyst thickness requirements preclude direct incorporation without transfer from the $\mathrm{Cu}$ growth surface to the target substrate. For a CMOS front end of the line (FEOL) application, where planar devices are formed and isolated, a growth and mechanical transfer process may be feasible, however, incorporation in back end of line (BEOL) applications will require direct incorporation as non-planar device components necessitate a transfer-free process. 
Research efforts to reduce both the required synthesis temperatures and necessary catalyst thicknesses to produce continuous graphene films are ongoing with examinations of alloy metal catalysts ${ }^{37}$, liquid phase precursors ${ }^{38}$, and the optimization of plasma enhanced CVD (PECVD) ${ }^{39}$ techniques, however a technique suitable for graphene inclusion in current CMOS technologies has not been demonstrated.

\subsection{1: The Chemical Vapor Deposition Mechanism}

As previously discussed, the majority of high quality and large area graphene is currently produced via CVD techniques with gaseous hydrocarbon precursors, micrometer scale $\mathrm{Cu}$ as both catalyst and substrate, and synthesis temperatures in excess of $800^{\circ} \mathrm{C}$.

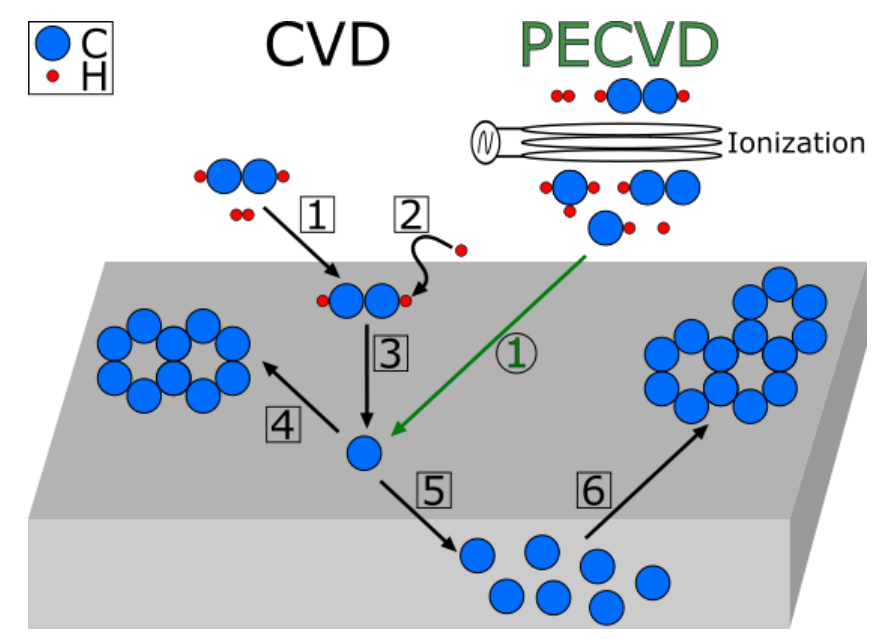

Figure 1.1: Comparison of 1) CVD and 1) PECVD techniques for graphene growth pathways on both low carbon solubility (1-4) and high carbon solubility (1-4 and 5-6) catalysts.

The typical CVD process (Fig. 1.1) occurs through introduction and adhesion of a hydrocarbon precursor to the target surface (Fig. 1.1: 1), dehydrogenation of the carbon precursor (Fig. 1.1:2) resulting in ionized carbon species on the target surface (Fig 1.1: 
3). From this point, the carbon species can participate in graphene formation (Fig 1.1:4) or, in the case of high carbon solubility catalysts, be absorbed into the material bulk (Fig 1.1: 5) and participate in graphene formation following precipitation upon cooling (Fig 1.1: 6). ${ }^{24}$ PECVD techniques represent an avenue to reduce required reaction temperatures through ionization of the hydrocarbon precursor prior to interaction with the targeted growth substrate (Fig 1.1: green 1) thereby reducing the energy required for dehydrogenation. ${ }^{25}$ This synthesis pathway represents a scalable method for graphene synthesis utilizing processes commonplace in the semiconductor industry, however, layer number control and synthesis of connected films below $600^{\circ} \mathrm{C}$ remains a challenge. One goal of this research project is to develop PECVD techniques, in conjunction with catalyst design, to reduce catalyst thickness and synthesis temperature requirements. In the following sections, a review of the current state of low temperature graphene synthesis will be discussed.

\subsection{2: Graphene Quality Determination via Raman Spectroscopy}

The reduction of graphene synthesis temperatures often results in the increased occurrences of defects in the film. These defects manifest as imperfections in graphene's network of $\mathrm{sp}^{2}$ hybridized carbon atoms in three primary ways: as 1) vacancies, due to either insufficient carbon availability or damage from high energy electron or ion interactions, 2) $\mathrm{sp}^{3}$ hybridized carbon molecules, due to incomplete dehydrogenation or doping, or 3) misaligned grain boundaries, due to numerous nucleation events resulting in graphene film formation from connections of multiple smaller domains. The types of 
defects, the defect concentration, and the layer number of a graphene film can be identified through characterization via Raman spectroscopy. ${ }^{40,41}$

Raman spectroscopy characterization occurs through detection of inelastic phonon-electron scattering events from exposure to an excitation laser. ${ }^{41,42}$ The Raman spectra for high quality graphene (Fig. 1.2) with $532 \mathrm{~nm}$ excitation is represented by three primary peaks, the $\mathrm{D}$ peak at $1350 \mathrm{~cm}^{-1}$, indicative of defects, the $\mathrm{G}$ peak at $1580 \mathrm{~cm}^{-1}$, and the 2D peak at $1680 \mathrm{~cm}^{-1}$, indicative of $\mathrm{sp}^{2}$ hybridization. ${ }^{43}$

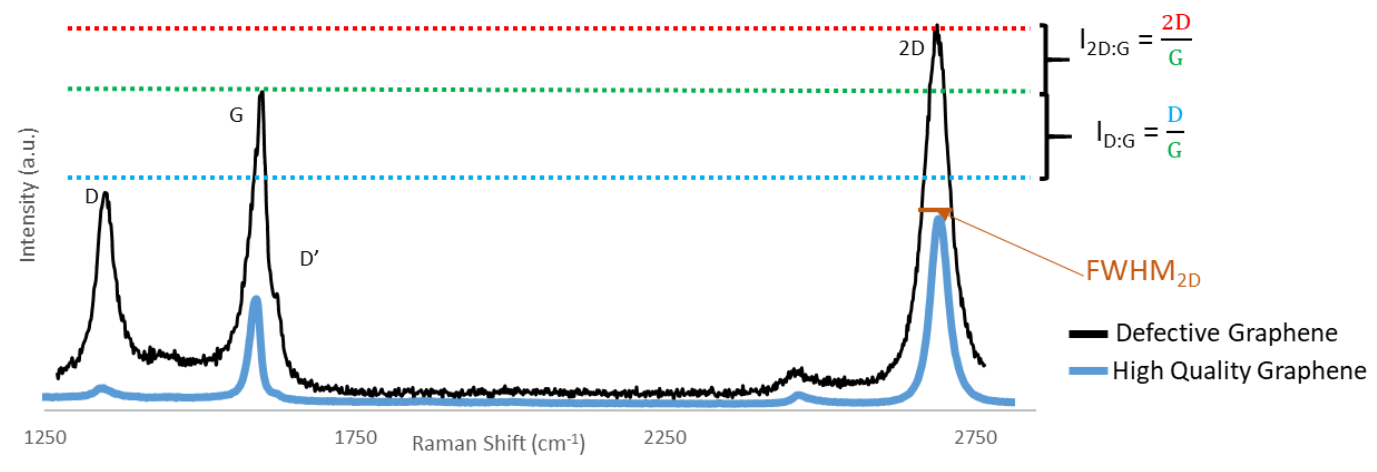

Figure 1.2: Typical Raman spectra detected from graphene displaying D, G, and 2D peaks. The ratios of these peak intensities are used to characterize graphene quality and layer number. In defective graphene, the D' peak is observed can be used to identify defect types.

The ratio of the intensities of the $2 \mathrm{D}$ peak to the $\mathrm{G}$ peak, $\mathrm{I}_{2 \mathrm{D} / \mathrm{G}}$, represents the layer number, with an increased intensity representative of fewer layers. Similarly, the full width half maximum of the $2 \mathrm{D}$ peak, $\mathrm{FWHM}_{2 \mathrm{D}}$, is another indicator of the number of graphene layers present, with $\mathrm{FWHM}_{2 \mathrm{D}}$ less than $30 \mathrm{~cm}^{-1}$ representative of monolayer graphene in pristine samples ${ }^{41}$ and $\mathrm{FWHM}_{2 \mathrm{D}}$ less than $45 \mathrm{~cm}^{-1}$ representative of monolayer graphene in defective, CVD graphene. Note that if multiple layers of graphene have basal, $\mathrm{AB}$, stacking, the number of layers can be determined through the 
number of Lorentzian functions necessary to fit the 2D peak, however, multiple layers of non- $\mathrm{AB}$ stacked graphene do not exhibit this property. ${ }^{40}$ The ratio of the intensity of the $D$ to $G$ peaks, $\mathrm{I}_{\mathrm{D} / \mathrm{G}}$, is indicative of defect densities present in the film with an increased ID/G representing increased defect occurrences. In highly defective graphene, the D' peak is observed and the ratio of intensities of $\mathrm{D}$ and $\mathrm{D}$ ' peaks, $\mathrm{I}_{\mathrm{D} / \mathrm{D}}$ ', can be used to identify the dominant defect types ( $\mathrm{sp}^{3}$, vacancy, or grain boundary) present within the film. ${ }^{44,45}$

Though the ultimate goal of research efforts around the globe is the identification of synthesis techniques resulting in pristine graphene formation on non-catalytic surfaces, the research presented here aims to identify graphene synthesis techniques suitable for CMOS and beyond-CMOS applications. In the case of diffusion barriers, connected graphene films with primarily $\mathrm{sp}^{3}$ type defects may outperform films with vacancies or grain boundary type defects. Additionally, there is evidence suggesting that targeted control of defect types and densities can provide an avenue to improve graphene spin transport properties. ${ }^{46}$ Raman spectroscopy, in conjunction with SEM inspection, is used to characterize the quality, layer number, and continuity of graphene films produced throughout this project.

\subsection{3: Challenges Associated with Low Temperature Graphene Synthesis}

Currently, most high quality and large area graphene is produced via CVD techniques with gaseous precursors, micrometer scale $\mathrm{Cu}$ as a catalyst and support, and synthesis temperatures in excess of $800^{\circ} \mathrm{C} .{ }^{26,29,47-49}$ Due to the relative thickness of the catalyst and elevated synthesis temperatures, these growths require a transfer process to 
the target substrate which limits incorporation of graphene to applications with only planar geometries. In recent years, significant research efforts have focused on reducing required synthesis temperatures and catalyst thicknesses with an ultimate goal of developing techniques for direct synthesis on substrates other than transition metal catalysts. ${ }^{50-53}$ An advancement in these synthesis techniques would eliminate damage and geometry related constraints associated with the transfer process while enabling direct incorporation of graphene in a variety of fields; from the semiconductor industry as an ultrathin diffusion barrier to the aerospace industry as lightweight strengthening and protective coatings. ${ }^{54-56}$ Researchers have identified three promising avenues towards this goal: the application of plasma enhanced CVD (PECVD) processes, the use of bimetal catalysts, and the choice of hydrocarbon precursor phase and configuration. Despite these advancements, control of graphene layer number and film connectivity remains a significant challenge as reaction temperatures and catalyst thicknesses are reduced. ${ }^{50}$ For example, PECVD techniques relying on ionization of the carbon precursor to reduce the energy required for graphene synthesis have yielded quality graphene at $600^{\circ} \mathrm{C}$ on predominantly copper $\mathrm{Cu} / \mathrm{Ni}$ alloys, however incomplete dehydrogenation and multilayer formation is observed upon further temperature reduction due to the reduced catalytic activity of the primarily $\mathrm{Cu}$ substrate ${ }^{57}$ Similarly, transition metals with partially filled D orbitals $(\mathrm{Fe}, \mathrm{Co}, \mathrm{Ni})$ have been identified as suitable candidates for CVD synthesis temperature reduction due to their increased ability for carbon ion stabilization, however, the increased carbon solubility in these metals leads to uncontrollable layer formation upon cooling. ${ }^{58-62}$ In attempts to alleviate this issue, $\mathrm{Ni}$ has been combined with less 
reactive metals, such as $\mathrm{Au}$, to suppress the formation of multilayer films through suspected passivation of the catalyst surface and reduction of carbon adsorption rates into the catalyst bulk, while reducing synthesis temperatures necessary to grow few-layer to monolayer films to $450^{\circ} \mathrm{C}$ following a $600^{\circ} \mathrm{C}$ anneal of the catalyst prior to growth. ${ }^{37}$ Though these results are promising, they require catalyst thicknesses of $500 \mathrm{~nm}$ or greater to minimize multilayer formation as well as elevated temperature catalyst pretreatments. In addition to the research efforts mentioned above, numerous gaseous carbon precursors, including methane, ethane, and propane, have been investigated and reveal that larger carbon precursor molecules allow graphene synthesis at reduced temperatures due to increased ion stability and reduced energy requirements for dehydrogenation. ${ }^{63,64}$ This trend has led to the development of CVD techniques employing solid phase and liquid phase carbon sources to further reduce required reaction temperatures for graphene synthesis through an increase in carbon precursor size. ${ }^{33,65,66}$ Graphene synthesis at $300^{\circ} \mathrm{C}$ has been performed with benzene and poly(methyl methacrylate) (PMMA) on $\mathrm{Cu}$ substrates, however $1000^{\circ} \mathrm{C}$ pretreatment of the catalyst is required prior to the synthesis. ${ }^{66,67}$ These results demonstrate the synergistic relationships among the carbon precursor molecule size, the ionization state, the target substrate reactivity, and the carbon solubility and thickness of the catalyst. Although graphene formation on low reactivity catalysts has been carried out through ionization of the hydrocarbon precursor and graphene growth on high reactivity catalysts has been achieved through both bimetal catalysts and increased hydrocarbon precursor sizes, techniques for in situ manipulation 
of carbon precursors tailored to the specific target substrate have not been thoroughly investigated.

Graphene synthesis at reduced temperatures has suffered from uncontrollable multilayer formation in the case of high carbon solubility thin-film catalysts or high temperature catalyst pretreatment requirements in the case of large carbon precursors. ${ }^{62,67}$ These problems stem from rapid catalyst saturation in the first case and reduced mobility of carbon species on the catalyst surface in the second. One avenue to overcome these challenges is a synthesis technique that utilizes both larger carbon precursor molecules to reduce the saturation rate of the catalyst bulk, and increased reactivity molecules to enable film completion between the nucleation locations of the larger molecules. Additionally, while bimetallic and alloy catalysts have shown promise for graphene synthesis temperature reduction and layer control, further optimization of morphology and composition is necessary for inclusion in industry applications.

\section{3: Graphene as a Spin Transport Channel}

While the primary effort toward graphene inclusion in current CMOS products revolves around reducing synthesis requirements to be compatible with existing components without degrading graphene's desirable characteristics that represent improvements over currently implemented materials, realization of graphene in beyondCMOS architectures does not suffer from this limitation. For these applications, high temperature synthesis is feasible due to graphene film inclusions as the first fabrication step, leading to the possibility of fabrication techniques benefitting from commercially 
available high quality graphene on the wafer scale. Recently, significant research activities have been focused on characterization of graphene as a spin transport channel for quantum computing and spintronic applications. These new technologies are necessitated as CMOS based interconnects with nanoscale dimension are facing tremendous challenges including the quantum limit, leakage, thermal constraints, signal/power integrity, and device parameter variability. These obstacles accelerate the need for new approaches to information storage and signal processing that would enable sustainable and functional scaling beyond the domain of CMOS ${ }^{68,69}$ Alternative options using spin-based phenomena, which exploit the spin freedom of electrons as carriers in electronic circuits, show promising merit to overcome these challenges. ${ }^{70-72}$

The field of spintronics, centered around injecting, manipulating, detecting, and optimizing the spin effects in electronic devices, is now witnessing intense interest following the discovery of the spin transfer torque effect. ${ }^{73-75}$ Graphene has remained at the forefront as a promising material candidate for long-distance communication and spin-logic nanotechnologies. ${ }^{76-83}$ With low intrinsic spin-orbit coupling and negligible hyperfine interaction, the predicted spin coherence length in graphene can extend up to a hundred micrometers, with spin lifetimes exceeding a microsecond.$^{82}$ In more than a decade of intensive investigations by a worldwide research community, the potential of graphene as an emerging material for spintronics has been established.

Experimental demonstrations of spin transport in graphene have been achieved by interfacing graphene with other classes of materials including ferromagnetic (FM) 
materials, semiconductors, and metal electrodes to enable spin manipulation, such as in the generation of pure spin current using non-local lateral spin valves or the control of magnetization in adjacent FMs through the spin torque phenomena. ${ }^{17,84-86}$ Researchers, using hexagonal boron nitride (h-BN) encapsulated exfoliated graphene as a transport channel in a nonlocal spin valve (NLSV), have detected a spin diffusion length of $12 \mu \mathrm{m}$ and a spin lifetime of 2 ns. ${ }^{85}$ Most recently, a group from Germany using a similar h$\mathrm{BN} /$ graphene encapsulation in the NLSV configuration, obtained a spin diffusion length of $30.5 \mu \mathrm{m}$ at room temperature and a spin lifetime of $12.6 \mathrm{~ns} .{ }^{87}$ Although these results are exciting, they are still well below the theoretically predicted graphene intrinsic limit, with uncertainty related to the dominant spin relaxation mechanism in graphene and spin interactions with other device components as likely responsible for the discrepancy. ${ }^{88,89}$ For graphene incorporation as a spin transport channel to be realized, new device designs must be developed to reveal graphene's intrinsic spin transport properties and enable the realization of spintronic platform technologies.

\subsection{1: Investigation of Graphene's Spin Transport Properties}

Pure spin currents transport only spins (spin angular momenta), unlike conventional spin-polarized currents, which carry both charges and spins. ${ }^{90}$ One wellknown method to generate a pure spin current is non-local spin injection. When spinpolarized current is injected from a ferromagnetic (FM) material into a non-magnetic (NM) material, spins are accumulated in the vicinity of the FM/NM interface. The accumulated spins can then diffuse in the NM material to form a pure spin current in which no charge current is present. ${ }^{91}$ The most commonly used device geometry for 
measuring graphene spin transport properties is the NLSV as shown in Figure 1.3. This NLSV consists of two metal contacts (C1 and C4) and two FM contacts (C2 and C3) sitting on top of a graphene ribbon serving as the spin transport channel. The contact C2 serves as a spin injector and C3 serves as a spin detector. The predominant advantage of a NLSV is that a pure spin current without charge flow can be generated. The measured spin signal is sensitive only to the relative orientations of spin populations at the detector and the spin accumulation at the injector, a signal that exhibits little background noise associated with charge current in a local configuration. The polarizations of the interfaces, spin diffusion lengths, and spin lifetimes can be determined by measuring the spin signals with varying injector and detector separations, and through a Hanle spin precession measurement with an applied transverse magnetic field. ${ }^{92}$

Utilizing this type of NLSV device, several groups have measured spin transport parameters in single layer graphene (SLG), bilayer graphene (BLG), and multilayer graphene (MLG). ${ }^{93-95}$ The pioneering work, done by the van Wees group from Zernike Institute for Advanced Materials in the Netherlands, demonstrated gate tunable spin transport and spin precession in SLG at room temperature. ${ }^{96}$ In that work, the electrical detection of spin precession was particularly important, as it proved the observed signals originated from spin transport and also suggested that the spin relaxation lengths were weakly dependent on charge density. Using similar NLSV devices, several other groups have carried out various investigations, including the measurement of anisotropic spin 


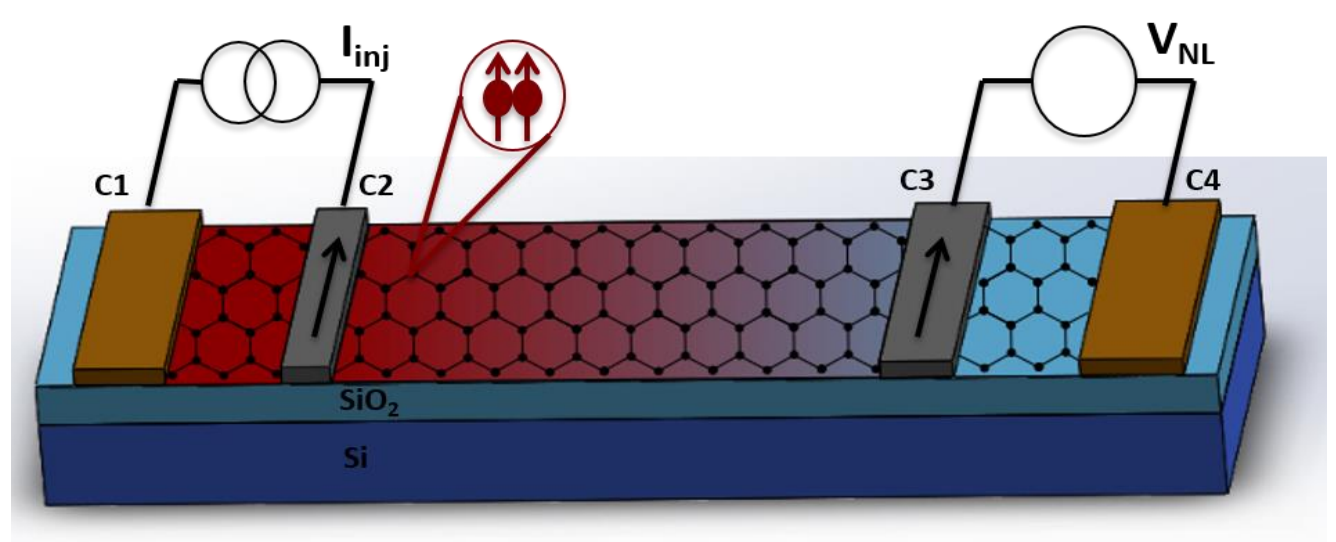

Figure 1.3: A schematic of the standard graphene NLSV in which spin injection at the FM injector (C2) results in spin polarized charge current traveling to the contact (C1), while spin diffusion occurs toward (C3) and is measured as a non-local voltage between the FM detector (C3) and contact (C4). The red to blue represents high to low gradient of $\mathrm{C} 1$ aligned spin concentrations.

relaxation ${ }^{97}$, local spin transport in MLG ${ }^{93}$, spindrift effects ${ }^{98}$, and bias dependence of spin injection. ${ }^{99}$ Although most of NLSV measurements have been on exfoliated graphene (known for its high quality), Kamalakar and co-workers from Sweden, reported using a NLSV channeled by Cu-foil-catalyzed CVD grown graphene to obtain spin transport and precession over long channel lengths up to $16 \mu \mathrm{m}$, a spin lifetime of $1.2 \mathrm{~ns}$, and a spin diffusion length $\sim 6 \mu \mathrm{m}$ at room temperature. ${ }^{84}$ These spin parameters are the highest for CVD graphene transferred to $\mathrm{SiO}_{2} / \mathrm{Si}$ substrates without using h-BN encapsulation, a fabrication process that is very challenging for industrial mass production due to the difficulty associated with acquiring large area h-BN thin films.

1.3.2: Challenges Associated with Measuring and Manipulating Graphene Spin Transport Although both theoretical calculations and experimental results are promising, the measurement and manipulation of graphene's spin transport properties are primarily carried out with a device similar to the one depicted in Figure 1.3, which will be referred 
hereafter as a "standard NLSV". To identify areas for graphene characterization improvements, it is important to understand how the standard NLSV works. In Figure 1.3, current is injected between the contacts, $\mathrm{C} 1$ and $\mathrm{C} 2$, and enables $\mathrm{C} 2$ to function as a spin injector, due to the reduced resistance experienced by electrons with spins aligned to the magnetic material through which they flow. ${ }^{17}$ During spin injection, spins accumulate in the channel underneath $\mathrm{C} 2$ and are transported in both directions, towards $\mathrm{C} 1$ (as a polarized charge current) and towards C3 (as a pure spin current). The pure spin current can then be detected by measuring the voltage across $\mathrm{C} 3$ and $\mathrm{C} 4$, with $\mathrm{C} 3$ as the spin detector. While a spin signal can also be detected between $\mathrm{C} 1$ and $\mathrm{C} 2$, it suffers from low signal to noise as the spin signal is small compared to the charge signal present between these two contacts. Instead, the nonlocal measurement of the pure spin current, or the signal between $\mathrm{C} 3$ and $\mathrm{C} 4$, arises only from the diffusion of spin-polarized electrons and exhibits increased signal to noise. This voltage $\left(\mathrm{V}_{\mathrm{NL}}\right)$ represents a non-equilibrium population of spins under $\mathrm{C} 3$, and is positive or negative depending on the relative magnetic orientation of $\mathrm{C} 2$ and $\mathrm{C} 3$, parallel (P) or antiparallel (AP). The relevant metric for spin transport is the difference in voltage between $\mathrm{C} 3$ and $\mathrm{C} 4$ when $\mathrm{C} 2$ and $\mathrm{C} 3$ are in parallel and antiparallel states, $\left\{\Delta \mathrm{V}_{\mathrm{NL}}=\left(\mathrm{V}_{\mathrm{NLP}}-\mathrm{V}_{\mathrm{NLAP}}\right)\right\}$, and it is often reported as a resistance through normalization by the injection current, $\mathrm{I}_{\mathrm{inj}}\left(\Delta \mathrm{R}_{\mathrm{NL}}=\Delta \mathrm{V}_{\mathrm{NL}} / \mathrm{I}_{\mathrm{inj}}\right)$. The $\Delta \mathrm{R}_{\mathrm{NL}}$ is the nonlocal resistance and represents a metric of spin signal that can be compared across devices with varied materials and configurations. In a standard NLSV, the contacts $\mathrm{C} 1$ and $\mathrm{C} 4$ are often made from non-magnetic but highly conductive metals, while C2 and C3 must be FM materials to enable injection and detection of spin population imbalances. 
This type of NLSV configuration has been broadly adopted for studying graphene spin-transport properties such as spin lifetime, spin diffusion length, and polarization injection efficiency; however, the measured graphene spin transport parameters are generally orders of magnitude smaller than those of theoretical predictions. Some reported hypotheses and proposed models describing spin scattering mechanisms and contactinduced dephasing by spin absorption in graphene are often inconsistent. To overcome these challenges, based on a broad literature survey, two critical issues have been identified, as reviewed below, that this research aims to address through novel device designs.

\subsection{3: The Role of FM Contacts on Spin Transport in a Standard Graphene NLSV}

One of the experimental roadblocks resulting in the discrepancy between theoretically predicted spin-transport parameters in graphene and experimentally measured parameters is the detrimental effect of FMs and their use as contacts. To elucidate these effects, several theoretical studies propose both spin scattering mechanisms, such as resonant spin scattering by magnetic impurities ${ }^{100}$, and the entanglement between spin and pseudospin by random spin orbit coupling ${ }^{101}$, which yield calculated spin lifetimes in the experimentally observed range. Some experimental and theoretical investigations demonstrate that the measured spin lifetimes are not intrinsic to graphene, but are rather limited by invasive contacts. ${ }^{89,91}$ Other studies suggest that spin re-absorption at the FM interface leads to reduced spin lifetimes and spin injection efficiencies due to the conductance mismatch between the FM contacts and the graphene channel ${ }^{102}$. Although the conductance mismatch problem can be alleviated 
with the insertion of a tunnel barrier (TB) at the spin injection interface ${ }^{100}$, fabricating uniform TB layers on top of graphene is non-trivial due to low surface energy and high surface diffusion leading to cluster formation. Recent developments in PVD fabricated tunnel barriers (TBs) have shown pinhole free barriers that can be fabricated through magnetron sputtering of $\mathrm{Al}$ followed by oxidation to form $\mathrm{Al}_{2} \mathrm{O}_{3} .{ }^{103}$ Additionally, examinations of other FM contact-induced effects, such as fringe fields, magnetic domain wall pinning, and current crowding at the FM spin injection interface reveal significant roles in spin relaxation. ${ }^{104,105}$

Researchers at UC Riverside systematically evaluated the roles of spin absorption, FM contact-induced effects, and bulk spin relaxation by analyzing Hanle spin precession data and compared traditional models (TMs), that do not explicitly take spin absorption into account, with spin adsorption models (SAMs), that account for spin relaxation through spin adsorption at the FM contact. ${ }^{106}$ This study concluded that SAMs better fit experimental data obtained from the contacts with TBs, suggesting that interface effects between FMs, TBs, and the graphene channel play primary roles in observed graphene spin transport parameters. Though improvements to TB and FM quality are important, characterizing their influence on measurements and maximizing their efficiency for manipulation of graphene spin properties calls for new device configurations.

\subsection{4: Hybrid Effect of Spin Drift and Diffusion in a Graphene NLSV}

Currently, spin lifetimes (up to $12.6 \mathrm{~ns}$ ) and spin diffusion lengths (up to $30.5 \mu \mathrm{m}$ ) are experimentally acquired by measuring Hanle spin precession in SLG using the 
standard graphene NLSV geometry. ${ }^{107}$ These experimental values are still significantly lower than those theoretically calculated, where several hundreds of micrometer spin diffusion lengths and a microsecond spin lifetime are predicted. ${ }^{83}$ The two most considered spin-relaxation mechanisms in metals and semiconductors are the ElliottYafet (EY) and D'yankonov-Perel' (DP) mechanisms. They are conventionally thought to be mutually exclusive, as EY occurs in materials with intact inversion symmetry, while DP is present in materials lacking or with broken inversion symmetry. ${ }^{108}$ Specifically, the EY mechanism describes a spin relaxation probability increasing with momentum relaxing collisions and the DP mechanism describes a spin relaxation rate inversely proportional to the momentum scattering rate. ${ }^{17}$ Though a comprehensive mechanism governing spin relaxation in graphene remains unidentified, the inverse dependence on momentum of EY and DP mechanisms has provided opportunities for spin momentum alteration to help clarify each mechanism's influence. Additionally, recent research indicates that spin and pseudo-spin interactions play an important role in spin relaxation in graphene, suggesting that resonant scattering mechanisms may contribute to discrepancies between theoretically predicted and experimentally observed spin lifetimes based on spin orbit coupling models. ${ }^{100,101}$ To take advantage of some of these mechanisms and to improve graphene spin transport properties, several groups have experimentally demonstrated that, by manipulating an external electrical field, graphene spin diffusion lengths and spin lifetimes can be extended. ${ }^{107,109,110}$ In 2016 , van Wees' group fabricated an h-BN encapsulated bilayer graphene (BLG) NLSV device to achieve spin diffusion lengths up to $90 \mu \mathrm{m}$ by adding a DC circuit between the spin injector and 
spin detector to enable carrier drift assisted diffusion spanning over unprecedented lengths. ${ }^{110}$ By introducing an external field that does not extend to the spin detector, the effect of charge carriers on pure spin transport can be realized without jeopardizing the signal reaching the spin detector. Although this device incorporates h-BN encapsulation of the graphene channel to minimize substrate effects leading to improved graphene spin transport, the effect of charge carrier interactions with pure spin signal resulting in enhanced spin transport parameters is demonstrated. These two phenomena, FM contact and charge carrier momentum influences on achievable spin lifetime and transport distances, represent areas for further characterization to enable graphene as a material component in spintronic devices.

\section{4: Problem Statement and Approach}

Graphene exhibits many properties that would represent a significant improvement in numerous industries. The lack of significant graphene incorporation in these industries stems from demanding synthesis requirements for uniform and connected films as well as a lack of fundamental mechanism descriptions for some properties that are necessary for implementation. The aim of this project is to develop graphene synthesis and characterization techniques to accelerate graphene inclusion in CMOS and beyond CMOS applications that would benefit from graphene's extraordinary mechanical and electrical properties. Two areas which continue to represent challenges for graphene inclusion have been identified: 1) synthesis temperature and catalyst requirements for the direct incorporation of connected graphene films and 2) characterization of spin transport 
and relaxation mechanisms in graphene transport channels to enable incorporation as an interconnect for spintronic applications.

To increase the viability of graphene incorporation in CMOS applications, graphene synthesis temperatures must be reduced to enable direct synthesis of graphene on targeted substrates. For direct inclusion in CMOS applications, the upper limit of this temperature range is $450-500^{\circ} \mathrm{C}$ to avoid mechanical damage to dielectric and metal materials. ${ }^{65}$ Additionally, direct inclusion will require catalyst free synthesis pathways or a significant reduction in required catalyst thickness such that the desired properties of graphene are not diminished by a catalyst layer 3-4 orders of magnitude larger than the resultant graphene film. Two techniques have been developed to reduce graphene synthesis temperature and catalyst requirements, 1) carbon precursor size and ionization manipulation targeted to the catalyst type during PECVD synthesis and 2) metal catalyst compositions tailored to reduce multilayer formation on thin film catalysts. These techniques are developed at $500^{\circ} \mathrm{C}$ with scalable catalyst deposition and graphene synthesis procedures amenable to inclusion in the current semiconductor industry.

The second goal of this research project, characterization of graphene spin transport properties, aims to increase the potential of graphene as an interconnect for spintronic devices. The majority of proposed spintronic applications, which utilize electron spin orientation, involve three primary phenomena, charge to spin conversion, spin manipulation and transport, and spin to charge conversion. ${ }^{71}$ Graphene, with weak SOC, represents a promising material candidate to alleviate a significant challenge to the 
advancement of this field: long distance and stable spin transport interconnects between logic operations. A review of the current literature has revealed two phenomena as areas for further characterization and optimization, spin relaxation due to ferromagnetic contact influences in standard NLSV devices and increased achievable spin transport distances through external electric fields. This research aims to investigate these phenomena through design, fabrication, and characterization of devices to identify ferromagnetic contact influences as well as determine the presence of momentum transfer from drift to diffusion elections in a graphene transport channel. A deeper understanding of these phenomena will enable further optimization of achievable graphene spin transport distances. Wafer scale device fabrication will be demonstrated with commercially available high quality graphene, produced with traditional high temperature CVD techniques, and industry standard lithography and metal deposition techniques to demonstrate scalability of graphene based devices. These graphene synthesis and spin transport results will be presented separately in the following two sections of this document. 


\section{2: Graphene Synthesis via Plasma Enhanced Chemical Vapor Deposition}

Results in this chapter have been published in the following reference:

Zietz, O.; Olson, S.; Coyne, B.; Liu, Y.; Jiao, J. Characterization and Manipulation of Carbon Precursor Species during Plasma Enhanced Chemical Vapor Deposition of Graphene. Nanomaterials 2020, 10, 2235. ${ }^{111}$

\section{1: Hydrocarbon Precursor Manipulation}

The choice of carbon precursor used during CVD and PECVD synthesis of graphene has been shown to significantly impact the ultimate quality and layer number of graphene produced. Presented in this section is a PECVD synthesis technique in which the size and ionization state of carbon precursor molecules reaching the growth catalyst is manipulated to reduce the rate of nucleation and absorption into the catalyst bulk, resulting in the formation of a continuous few-to-monolayer graphene film at $500^{\circ} \mathrm{C}$. This is achieved through control of the inlet between a remote inductively coupled plasma (ICP) location and the catalyst location that enables both ion screening and secondary capacitively coupled plasma $(\mathrm{CCP})$ generation. Improvements for the controlled growth achievable with this precursor screening technique is demonstrated on a $50 \mathrm{~nm}$ thick $\mathrm{Ni} / \mathrm{Cu}$ catalyst in which $2 \mathrm{wt} \% \mathrm{Cu}$ was used. This catalyst thickness represents a 10-fold reduction compared to previously published results and allows us to eliminate the elevated temperature pre-growth anneal required by previous reports. ${ }^{37}$ Characterization of the generated plasma species is performed via UV-Vis inspection, while mass spectrometer (MS) characterization of the growth chamber coupled with current monitoring at the catalyst location enables identification of species reaching the catalyst. It is observed that the layer number and defect concentrations can be controlled via ion 
screening processes, while a secondary ionization procedure leads to further reduction in both defect concentrations and multilayer portions of the film.

\subsubsection{Introduction}

To develop a synthesis technique for enhanced control of graphene film quality and uniformity at reduced temperatures, toward inclusion in CMOS applications, a systematic characterization and manipulation of hydrocarbon precursors generated during plasma enhanced chemical vapor deposition of graphene was performed. Remote ionization of acetylene was observed to generate a variety of neutral and ionized hydrocarbon precursors, while in situ manipulation of the size and reactivity of species permitted to interact with the growth catalyst enabled control of the resultant graphene morphology. Selective screening of high energy hydrocarbon ions coupled with a multistage bias growth regime resulted in synthesis of $90 \%$ few to monolayer graphene on $50 \mathrm{~nm} \mathrm{Ni} / \mathrm{Cu}$ alloy catalysts at $500^{\circ} \mathrm{C}$. Additionally, synthesis with low power secondary ionization processes were performed and reveal further control during the growth, resulting in a 50\% reduction in average defect densities throughout the film. Mass spectrometry and UV-Vis spectroscopy monitoring of the reaction environment in conjunction with Raman characterization of the synthesized graphene films enables correlation of the carbon species permitted to reach the catalyst surface to the ultimate quality, layer number, and uniformity of the graphene film. These findings reveal a robust technique to control graphene synthesis pathways during plasma enhanced chemical vapor deposition. 


\subsubsection{Results and Discussion}

All the experimental results are obtained in custom-built reactor as shown in

Figure 2.1 a that displays a schematic of the reaction chamber, with remote ICP location and configurable inlet along the path from the plasma to the catalyst. A positive or

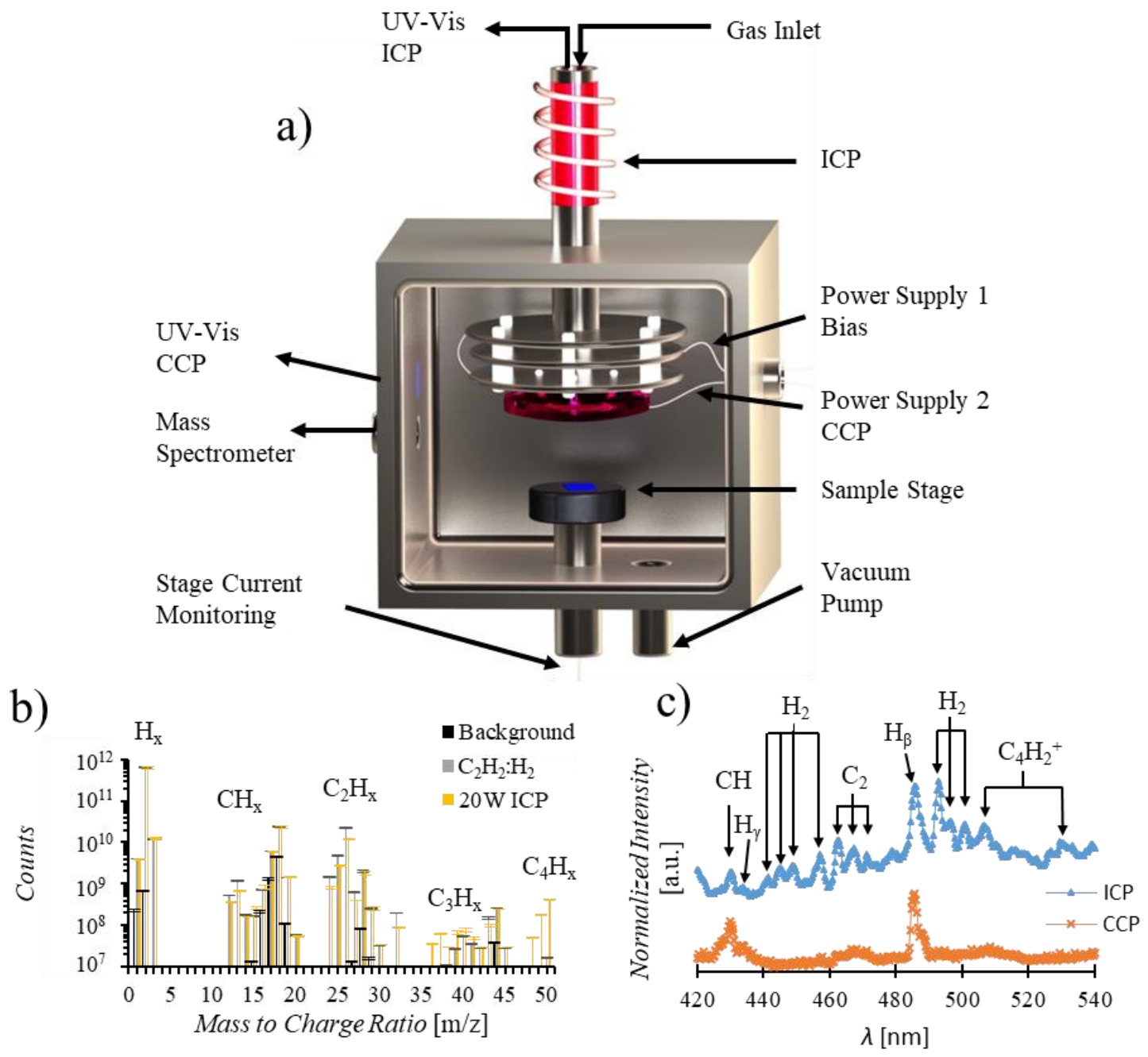

Figure 2.1: a) Schematic of custom plasma enhanced CVD (PECVD) system with configurable inlet enabling ion screening and secondary plasma generation as well as stage current monitoring, mass spectrometry, and UV-Vis spectroscopy. (b Mass spectrum indicating chamber background (in black) composition is primarily $\mathrm{H} 2 \mathrm{O}$ and $\mathrm{CO} 2(\mathrm{~m} / \mathrm{z} 18,28,44)$. Gas introduction, $\mathrm{C} 2 \mathrm{H} 2: \mathrm{H} 2$ in a $0.1: 15$ ratio (displayed in grey), results in increased detection of 1 and 2 carbon containing species (m/z 13-16, 24-26) while 20W inductively coupled plasma (ICP) ignition (displayed in yellow) results in the detection of 3 and 4 carbon species ( $\mathrm{m} / \mathrm{z}$ 36-39, 47-50). (c UV-Vis spectrum collected for a 20W ICP (shown in blue) and a $2.5 \mathrm{~W}$ capacitively coupled plasma (CCP) (shown in orange) indicate the increased diversity of both hydrogen and carbon signals present at the higher powered ICP while primarily $\mathrm{H} \beta$ and $\mathrm{CH}$ ionization events occur in the low power CCP. 
negative voltage can be applied to the inlet plates independently to screen ions and/or generate a secondary CCP. Current monitoring at the sample stage enables characterization of the inlet plate effects on charged species reaching the catalyst. Monitoring of the growth chamber via mass spectrometry permits identification of neutral species reaching the catalyst location through analysis of fragments generated upon ionization at the detector. Ionized species generated in the plasma are not expected to reach the MS which is separated from the main chamber by a leak valve. This is verified by a lack of signal detected when the ionizing component of the MS is turned off in the presence of plasma at the ICP or CCP location. As depicted in Figure $2.1 \mathrm{~b}$, the background composition of the chamber at $1 \times 10-7$ torr is primarily $\mathrm{H}_{2} \mathrm{O}$ and $\mathrm{CO}_{2}$. Introduction of $\mathrm{C}_{2} \mathrm{H}_{2}$ and $\mathrm{H}_{2}$ results in an expected increase in 1 and 2 carbon species while ignition of a $20 \mathrm{~W}$ plasma at the remote ICP location results in the generation of 3 and 4 carbon species, in agreement with previously reported characterizations of acetylene plasmas. ${ }^{112-114}$ Figure $2.1 \mathrm{c}$ displays the UV-Vis spectrum collected at the ICP and CCP locations, confirming the generation of these larger hydrocarbon molecules with the presence of a plasma. Characterization of gaseous species generated both at the remote ICP location and those that reach the mass spectrometer reveal that there is an increase in ionization events (Fig. 2.2: a), and a reduction in neutral species reaching the MS detector (Fig. 2.2: b) with increasing remote plasma power. However, plasma power variation alone does not enable selection for carbon precursor size. Additionally, current measurements at the catalyst location during remote plasma operation confirm that primarily positive ionic species are reaching the catalyst and that the application of a 
negative bias to a reaction chamber inlet plate effectively blocks these ions from reaching the catalyst (Fig. 2.2: c).
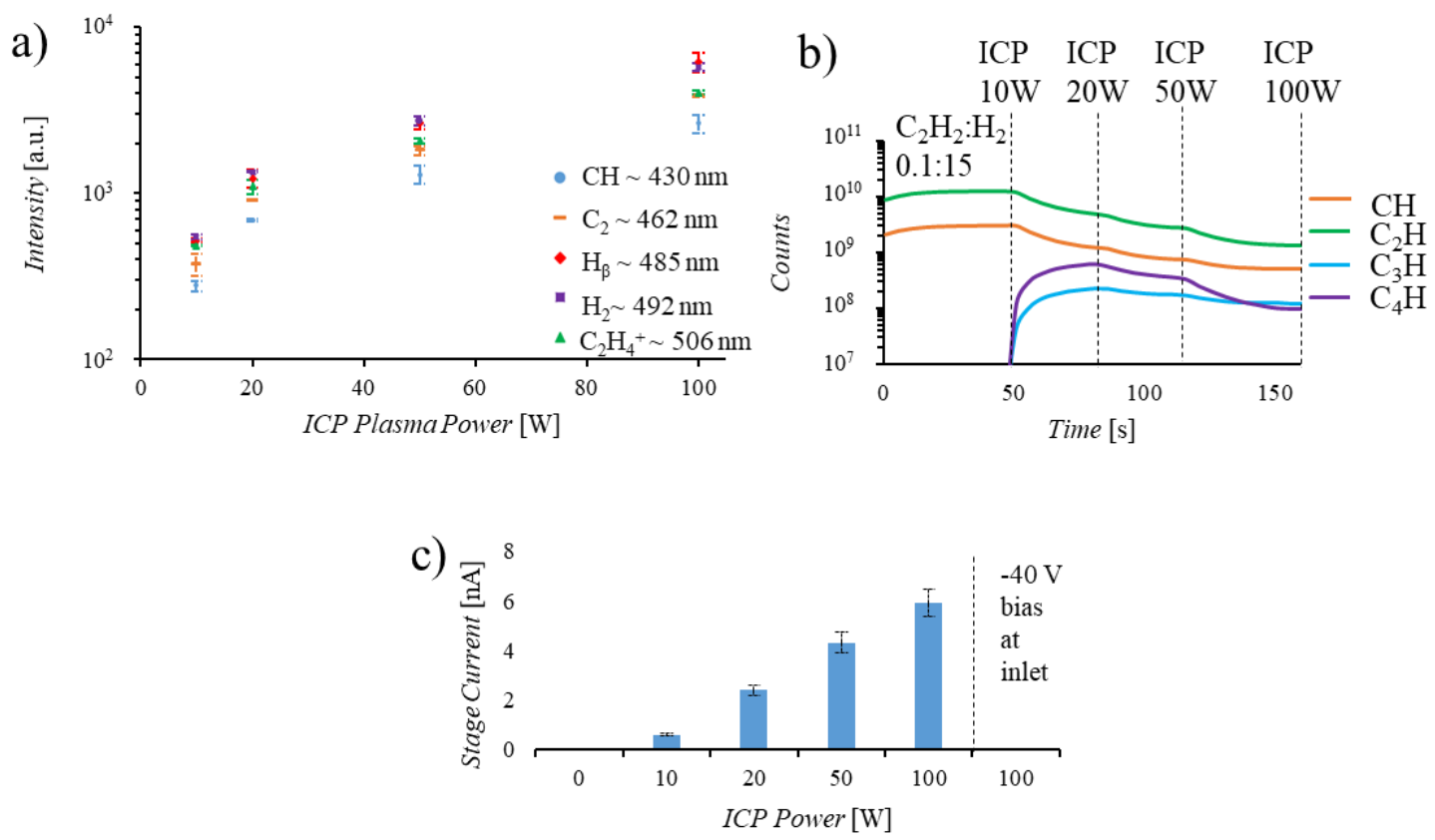

Figure 2.2: a) UV-Vis spectrum at varied ICP powers indicate that increasing plasma power results in an increased occurrence of ionization events for all species. b) Mass spectrum displaying the increase of 3 and 4 carbon species with plasma ignition and the reduction of all detected species as plasma power increases, indicating that fewer neutral species are reaching the detector as ICP power is increased. c) Stage current readings displaying an increase in stage current as ICP power is increased and zero current detected when a screening bias is applied at the chamber inlet, displaying effective reduction of charged species reaching the stage.

These results indicate that, while increasing remote plasma power alone does not enable significant selectivity for the size of species generated, the average size of carbon precursors reaching the catalyst can be increased through remote plasma operation coupled with screening of high energy ions through the application of a negative bias at a chamber inlet plate.

To identify the effects of in situ precursor manipulation on achievable graphene quality, all reported synthesis was performed in the custom reactor with $50 \mathrm{~nm} \mathrm{Ni} / \mathrm{Cu}$ 
catalysts, $2 \mathrm{wt} \% \mathrm{Cu}$, at $500^{\circ} \mathrm{C}$ for 1 minute and $\mathrm{C}_{2} \mathrm{H}_{2}: \mathrm{H}_{2}$ precursor flow rates of 0.1 $\mathrm{sccm}: 15 \mathrm{sccm}$. Following transfer of the graphene films, Raman mapping is performed to characterize quality and uniformity with ratios of the intensity of $\mathrm{D}, \mathrm{G}$, and $2 \mathrm{D}$ bands as well as the full width at half maximum (FWHM) of the 2D peak to determine the layer number and defect density of the films. Fewer layers are present with increasing $\mathrm{I}_{2 \mathrm{D} / \mathrm{G}}$, and defect densities increase with increasing $\mathrm{I}_{\mathrm{D} / \mathrm{G}}$. While pristine monolayer graphene displays a nearly undetectable $\mathrm{I}_{\mathrm{D} / \mathrm{G}}$ and an $\mathrm{I}_{2 \mathrm{D} / \mathrm{G}} \geq 2$, when defects are present monolayer graphene is identified by an $\mathrm{I}_{2 \mathrm{D} / \mathrm{G}}>1$ and $\mathrm{FWHM}_{2 \mathrm{D}}<100 \mathrm{~cm}^{-1} .115,116$ To categorize areas of multilayer and monolayer graphene in these samples, 2D maps of $\mathrm{I}_{2 \mathrm{D} / \mathrm{G}}$ are presented with color scales fixed between 1 and 2 , with black areas, $\mathrm{I}_{2 \mathrm{D} / \mathrm{G}} \leq 1$, representing multilayer portions of the film, white areas, $\mathrm{I}_{2 \mathrm{D} / \mathrm{G}} \geq 2$, representing low defect density monolayer portions of the film, and orange areas, $1<\mathrm{I}_{2 \mathrm{D} / \mathrm{G}}<2$, representing few-tomonolayer portions of the film. Figure 2.3 a and b display a $100 \mathrm{um}^{2} \mathrm{I}_{2 \mathrm{D} / \mathrm{G}}$ Raman map, with accompanying average Raman spectrum for the mapped area, of samples synthesized with and without an applied screening bias at the inlet plate respectively. It is observed that with the application of a screening bias, both average layer number and areas of multilayer (areas with $\mathrm{I}_{2 \mathrm{D} / \mathrm{G}}<1$ indicated by black portions of the Raman map) are reduced compared to the unscreened case by $62 \%$. The reduction of multilayer portions of the film under the applied bias condition is attributed to the screening of high energy ions that are more readily dehydrogenated and adsorbed into the catalyst bulk, leading to rapid saturation and multilayer formation upon cooling. While these ions are screened by the applied bias, the neutral molecules, including 3 and 4 carbon species $(\mathrm{m} / \mathrm{z}$ 
$36-39,47-50)$ generated in the remote plasma, are permitted to reach the main reaction chamber and participate in graphene formation at the catalyst surface. Though a significant reduction in multilayer portions is observed, the graphene film remains highly
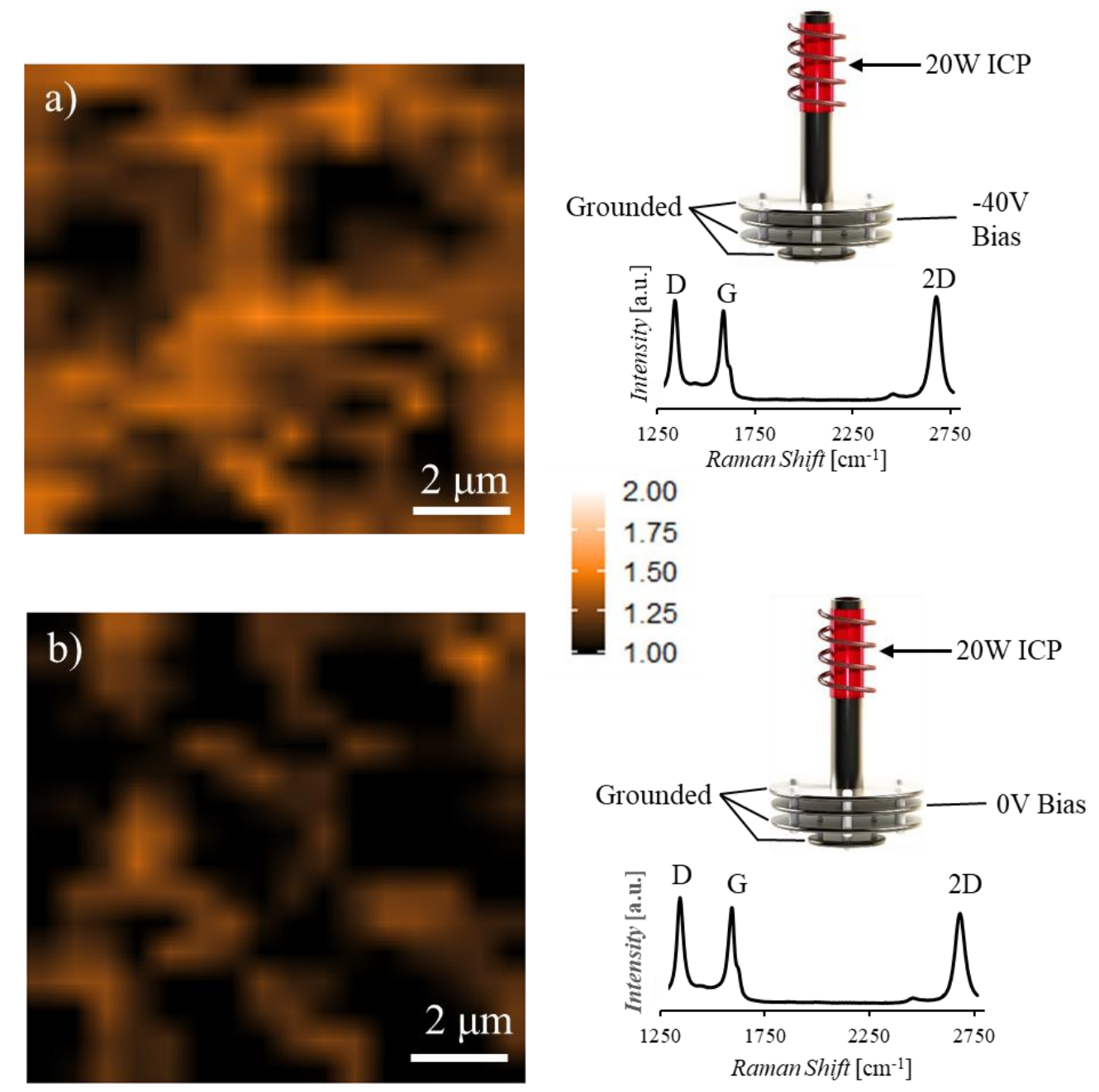

Figure 2.3: Ion screening bias effects on graphene film layer number. $100 \mu \mathrm{m}^{2}$ Raman $\mathrm{I}_{2 \mathrm{D} / \mathrm{G}}$ map and accompanying average Raman spectrum over the mapped area for graphene samples synthesized with 20W ICP and a) -40V screening bias applied at the chamber inlet and b) no applied screening bias during the 1-minute synthesis. Increased multilayer formation (black portions of the mapped area) is observed on the unscreened case when compared to the biased case while both average Raman spectrums indicate elevated defect concentrations, with average $\mathrm{I}_{\mathrm{D} / \mathrm{G}}>1$.

defective. The films (Fig. 2.3: a and b) have an average $\mathrm{I}_{\mathrm{D} / \mathrm{G}}$ of 1.2, with an increased background between the $\mathrm{D}$ and $\mathrm{G}$ peaks indicative of remaining $\mathrm{sp}^{3}$ hybridization through C-H bonds. ${ }^{117}$ 
Synthesis results under the biased plate condition indicate that to reduce the layer number and defect densities of the graphene films, both a reduction in nucleation density and an increase in dehydrogenation rates must be achieved. To characterize the capability of this ion screening technique toward achieving these goals, multistage growths were performed in which the screening bias was applied for a portion of the synthesis. Figures 2.4 a and b display Raman maps and accompanying average Raman spectrum from samples in which the bias was applied for the first or second half of the 1minute synthesis, respectively. The synthesis performed with a screening bias for the first 30s of the growth (Fig. 2.4: a) displays a small increase in multilayer coverage when compared to the synthesis with bias application for the growth entirety (Fig. 2.3: a).

This result indicates that the initial screening of high energy ions results in nucleation occurring primarily from neutral and larger carbon containing species and the removal of the screening bias allows high energy ions to reach the catalyst and continue both growth at the surface and saturation of the bulk. Conversely, the sample produced with a screening bias applied for the second $30 \mathrm{~s}$ (Fig. 2.4: b) displays a significant increase in multilayer formation indicating high rates of nucleation, growth, and absorption into the catalyst bulk during the initial $30 \mathrm{~s}$ where no screening bias is applied. Application of the screening bias during the final 30s of the synthesis removes the ionized species responsible for dehydrogenation and film completion, resulting in 
increased multilayer formation. Further reduction in multilayer formation and defect density (Fig. 2.4: c) is achieved through application of the bias for the first 30s of the
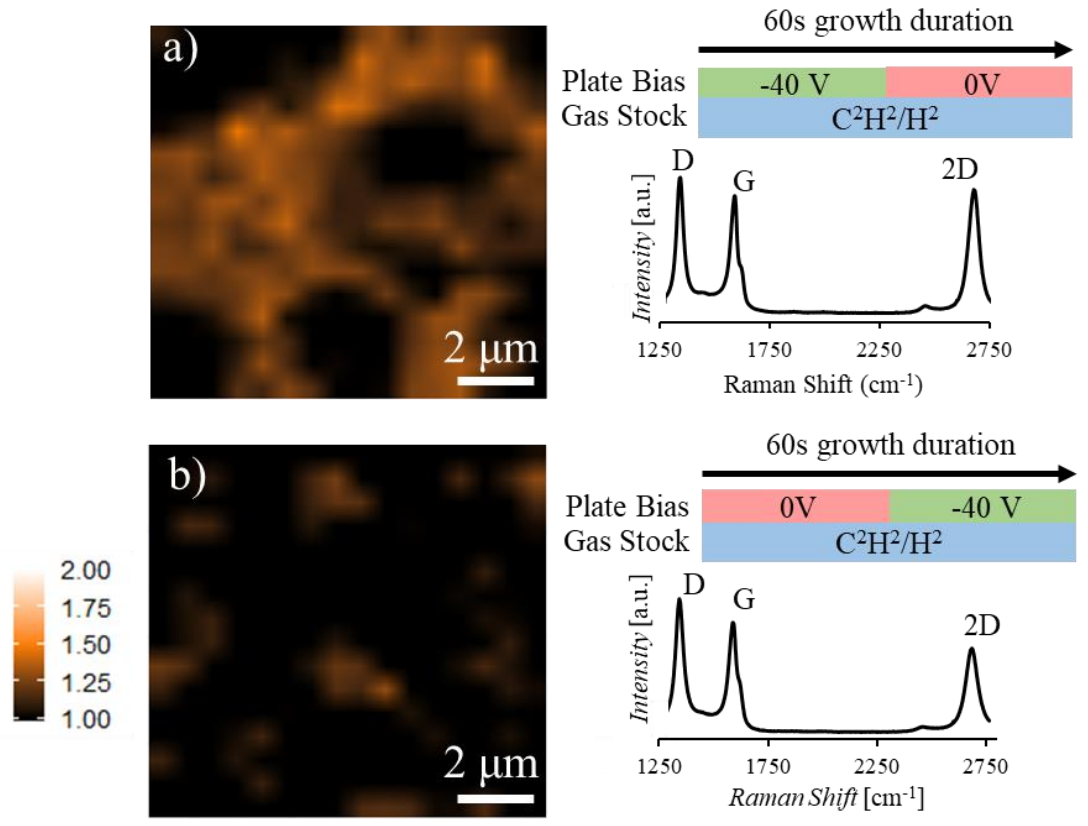

Gas Stock
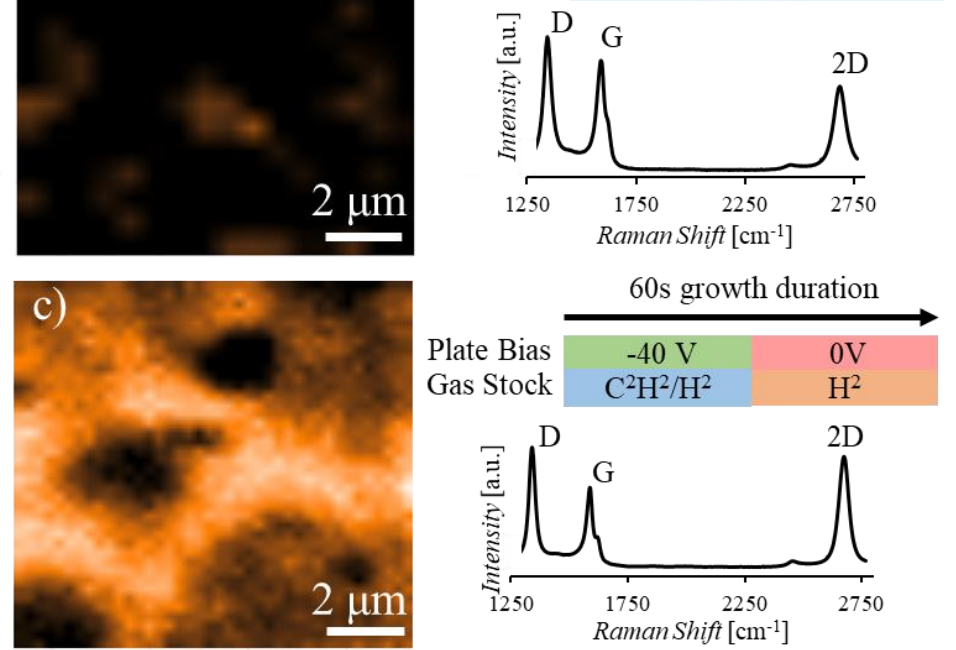

Gas Stock
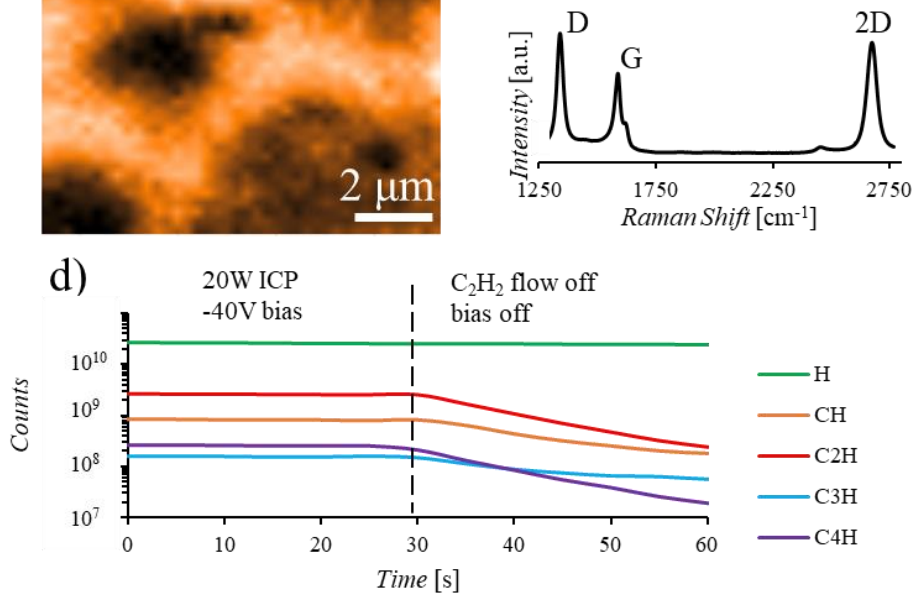

Figure 2.4: Multistage graphene synthesis with ion screening bias application and carbon precursor presence at differing portions of the growth. $100 \mu \mathrm{m}^{2}$ Raman map and accompanying average Raman spectrum over the mapped area for graphene samples synthesized with $20 \mathrm{~W}$ ICP for 1 minute and a) $-40 \mathrm{~V}$ screening bias for the first $30 \mathrm{~s}$ of synthesis, b) $-40 \mathrm{~V}$ screening bias applied for the second $30 \mathrm{~s}$ of synthesis, and c) $-40 \mathrm{~V}$ screening bias applied for the first $30 \mathrm{~s}$ of synthesis and both the bias and $\mathrm{C}_{2} \mathrm{H}_{2}$ feedstock removed for the second $30 \mathrm{~s}$ of synthesis.

synthesis followed by removal of both the bias and the carbon precursor feed stock to the 
remote plasma location for the second half of the synthesis (Fig. 2.4: d). This results in reduced nucleation rates during the initial stage of the growth, associated with bias application, and, with the removal of both the bias and the carbon feedstock, increased rates of dehydrogenation without continued layer formation during the second half of the synthesis. This multistage ion screening synthesis technique enables production of continuous and predominantly few-to-monolayer, $91 \% \mathrm{I}_{2 \mathrm{D} / \mathrm{G}}>1$, graphene at $500^{\circ} \mathrm{C}$ without requiring an increased temperature anneal.

Further control over the reactivity of species reaching the catalyst location can be achieved through the generation of a low power, $2.5 \mathrm{~W}$, secondary plasma after the ion screening location. Figure 2.5 a shows a Raman map and average Raman spectrum of graphene produced during a 1-minute synthesis with both a remote plasma and a secondary plasma, representing a significant reduction in average defect densities, from 1.4 to $0.7 \mathrm{I}_{\mathrm{D} / \mathrm{G}}$, while increasing few-to-monolayer coverage, $95 \% \mathrm{I}_{2 \mathrm{D} / \mathrm{G}}>1$. MS characterization (Fig. 2.5: b) of the reaction environment indicates a reduction in 3 and 4 carbon species with the ignition of a secondary plasma while the concentration of 1 and 2 carbon species remains relatively unaffected. Additionally, UV-Vis monitoring of the secondary $\mathrm{CCP}$ (Fig. 2.5: c) reveals that primarily $\mathrm{H}$ ionization events occur when the remote $\mathrm{ICP}$ is present while both $\mathrm{H}$ and $\mathrm{CH}$ ionization events occur with only the secondary CCP (Fig. 2.1: c). These results, coupled with the detection of a current at the sample location upon ignition of the secondary $\mathrm{CCP}$, indicate that 3 and 4 carbon species generated in the 20W ICP are not reaching the MS and may be the primary species ionized at the secondary CCP location prior to interacting with the catalyst. 

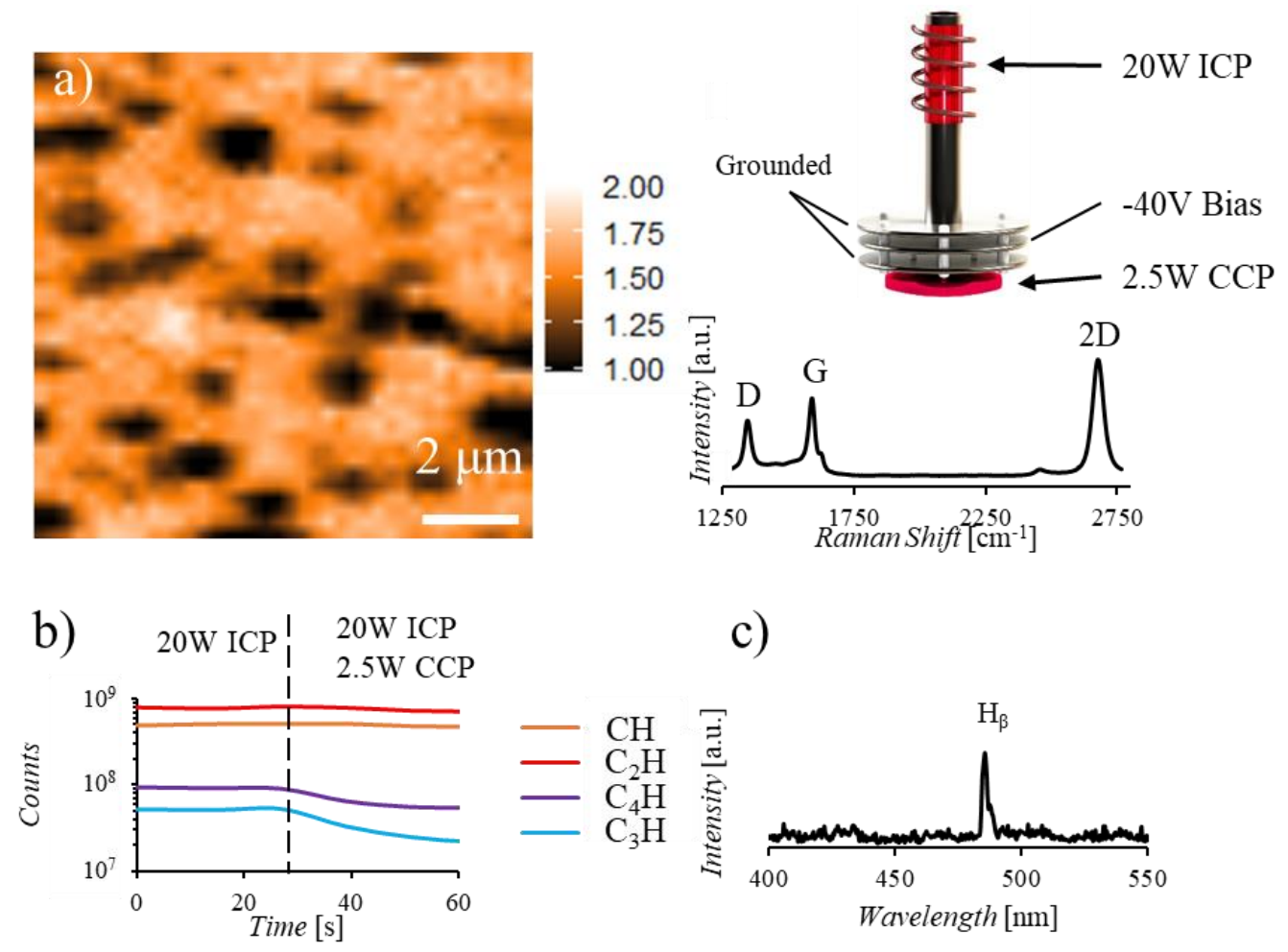

c)

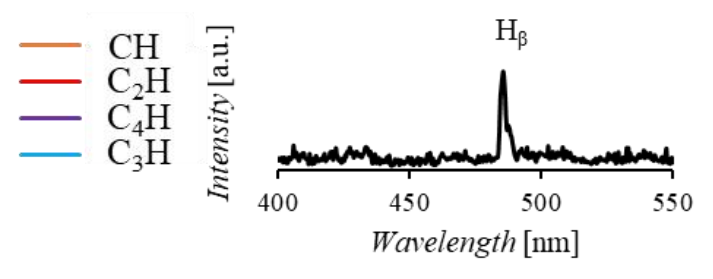

Figure 2.5: Graphene synthesis with both ICP and secondary CCP resulting in reduced layer number and defect density. Raman map, a), indicating primarily monolayer formation $\left(95 \% \mathrm{I}_{2 \mathrm{D} / \mathrm{G}}>1\right)$ and accompanying average Raman spectrum displaying reduced defect densities compared to multistage synthesis results in Figure 4. b) Mass spectrum depicting the change in hydrocarbon species present with the ignition of a secondary CCP. Note that the number of 3 and 4 carbon species is reduced with ignition of the secondary plasma while the number of 1 and 2 carbon species remains nearly constant. c) UV-Vis spectrum of CCP collected while ICP plasma generation is also occurring, indicating primarily $\mathrm{H}$ ionization. Note the reduction in $\mathrm{CH}$ and $\mathrm{C}_{2}$ ionization events compared to the $\mathrm{CCP}$ spectrum, Fig. 2.1: c, collected when no upstream ICP is present.

Comparing the Raman map under this two-plasma, ICP and CCP, condition (Fig. 2.5: a) to the map of the sample synthesized under a multistage bias condition (Fig. 2.4: c), an increased number but decreased size of multilayer islands is observed in the two-plasma case. We hypothesize that is phenomena results from an increased nucleation rate associated with larger carbon precursors which are generated at the ICP location and ionized at the CCP location before reaching the catalyst. These larger ionized species are more likely to nucleate at the catalyst surface, resulting in the increased number of 
multilayer islands observed, but are less likely to be absorbed into the catalyst bulk, resulting in the overall increase in few-to-monolayer content of the film. While bias application alone screens high energy ions and a multistage bias synthesis condition reduces multilayer formation, this secondary ionization technique increases few-tomonolayer coverage to $95 \%$ through both increasing the reactivity of carbon precursors and reducing the rate of catalyst saturation.

This phenomenon of controlling the concentration and ionization states of precursor molecules permitted to interact with the growth substrate has resulted in the significant increase in few-to-monolayer coverage in the secondary bias case. While the dependence on carbon species size and ionization state has been demonstrated, the specific roles of each ionized species within the larger groups, i.e., 3 carbon and 4 carbon species, will require in situ characterization of reactions occurring at the catalysts surface. Future work in this area should lead to improvements in targeting specific precursor species to intended substrates and continue to advance efforts toward graphene inclusion in a variety of fields.

\subsubsection{Summary}

In summary, we have demonstrated graphene synthesis techniques utilizing the in situ manipulation of carbon precursors generated during plasma enhanced chemical vapor deposition to achieve continuous graphene films at reduced temperatures on reduced catalyst thicknesses. This technique, which is not represented in literature, has been found to enable manipulation of nucleation density, layer number, and defect densities though control of carbon precursor sizes and ionization states. Screening bias application 
between a remote ionization location and the sample location enables targeting of larger neutral molecules while a secondary ionization event can increase the reactivity of these molecules. We have utilized this technique to demonstrate 95\% few-to-monolayer graphene synthesis on $50 \mathrm{~nm} \mathrm{Ni} / \mathrm{Cu}$ thin film catalysts with average $\mathrm{I}_{\mathrm{D} / \mathrm{G}}$ of 0.7 at $500^{\circ} \mathrm{C}$, without the need for any high temperature catalyst pretreatments. This technique represents not only an avenue for continued reduction of synthesis temperature and transition metal catalysts thickness requirements but reveals a novel method for active species control in broader PECVD synthesis techniques.

\subsubsection{Experimental Methods}

Catalyst Deposition and Graphene Synthesis

$50 \mathrm{~nm} \mathrm{Ni} / \mathrm{Cu}$ catalysts were deposited on $\mathrm{Si} / \mathrm{SiO}_{2}$ wafers through magnetron sputtering (Kurt J. Lesker AXXIS) of $48 \mathrm{~nm} \mathrm{Ni}$ followed by $2 \mathrm{~nm} \mathrm{Cu}$ without breaking vacuum. Graphene synthesis was performed in the custom PECVD chamber initiated by chamber evacuation to base pressure of $1 \times 10^{-7}$ torr followed by heating to $500^{\circ} \mathrm{C}$ under $15 \mathrm{sccm}$ of $\mathrm{H}_{2}$, resulting in a chamber pressure of 50 mtorr. To promote cleaning and alloying of the catalyst, the sample was held at $500^{\circ} \mathrm{C}$ for 2 minutes under $\mathrm{H}_{2}$ flow prior to introduction of the hydrocarbon precursor. Graphene growth was initiated by introduction of $\mathrm{C}_{2} \mathrm{H}_{2}$ at $0.1 \mathrm{sccm}$ and ignition of a $20 \mathrm{~W}$ ICP plasma for 1 minute. Screening bias and secondary CCP were applied according to the desired synthesis regime through a $-40 \mathrm{~V}$ bias application (Glassman PSFX) to the first inlet plate or CCP generation at $2.5 \mathrm{~W}$ (Bertan 205A) with a negative bias applied to the second plate. Following completion of the synthesis regime, ICP, CCP, and screening bias power was 
set to zero, as well as the $\mathrm{C}_{2} \mathrm{H}_{2}$ flow rate. Finally, the sample was allowed to cool under $15 \mathrm{sccm} \mathrm{H}_{2}$ until $150^{\circ} \mathrm{C}$ over approximately 15 minutes before removal from the chamber.

Reaction Chamber Characterization

$\mathrm{UV}-\mathrm{V}$ is characterization was performed through spectrum collection (Ocean Optics USB200+) of ICP and CCP signals through isolated viewports, above the ICP and on the main chamber for CCP. Stage current characterization was performed through Pico ammeter (Keithley 485) monitoring of the sample stage. Mass spectrometry (Pfeiffer PrismaPro QMG 250 M2) was collected in a secondary chamber, with differential pumping to maintain $1 \times 10^{-6}$ torr, which is connected to the main chamber through a leak valve.

Graphene Transfer and Characterization

Graphene was transferred from the catalyst through spin coating (Laurel WS-650) $300 \mathrm{~nm}$ polymethyl methacrylate (PMMA) support and baking in air at $150^{\circ} \mathrm{C}$ for 5 minutes. The sample was submerged in $0.5 \mathrm{M} \mathrm{FeCl}_{3}$ to etch both $\mathrm{Ni}$ and $\mathrm{Cu}$ until the graphene/PMMA floated to the surface. Following 5 rinses for 1 min each in DI water, the graphene with PMMA support was transferred to fresh $\mathrm{Si} / \mathrm{SiO}_{2}$ and PMMA was removed in acetone. Raman Characterization was performed on a Horiba Jobin Yvon HR800 with $532 \mathrm{~nm}$ laser excitation and mapping acquisition capabilities through a motorized sample stage. Raman map characterization and spectrum averaging were 
performed through in-house software, written in R, to peak fit D, G, and 2D bands for each spectrum collected.

\section{2: Catalyst Design and Characterization}

Results in this chapter have been published in the following references:

Zietz, O.; Olson, S.; Coyne, B.; \& Jiao, J. Increased Sample Yield and Achievable Imaging Resolutions Through Thin Film Transfer Technique. Microscopy and Microanalysis 2018, 24(S1), 1630-1631. ${ }^{118}$

Zhan, H.; Jiang, B.; Zietz, O.; Olson, S.; Jiao, J. Simulation to fabricationunderstanding the effect of $\mathrm{NiAuCu}$ alloy catalysts for controlled growth of graphene at reduced temperature. Materials Research Express 2019, 7, 015603. ${ }^{119}$

In addition to carbon precursor manipulation, alloy and bimetallic catalysts have shown great promise for modulation of graphene synthesis pathways and achievable graphene film sizes, layer numbers, and quality. For graphene synthesis temperature reduction, $\mathrm{Ni}$ is commonly employed due to its increased ability for carbon dehydrogenation and inhomogeneous and multilayer graphene films can be formed at temperatures low as $450^{\circ} \mathrm{C}$. While NiAu catalysts have been shown in literature to reduce layer formation and $\mathrm{NiCu}$ catalysts were demonstrated to enable primarily few-tomonolayer graphene synthesis in the preceding section, optimization of catalyst composition remains an avenue for further advancement in graphene synthesis techniques. ${ }^{120}$ To identify catalyst candidates for graphene layer number reduction and increased uniformity, bulk Ni catalysts with small weight percentages of both $\mathrm{Au}$ and $\mathrm{Cu}$, that is trimetallic $\mathrm{NiAuCu}$ catalysts, are characterized and compared to bimetallic catalysts of NiAu and NiCu. Graphene synthesis via PECVD is performed on various compositions of these catalysts and electron backscatter diffraction (EBSD) characterization of the catalyst morphology in conjunction with simulation of the 
required energy for acetylene decomposition on these multimetal catalysts reveals avenues for graphene quality and layer number control. These results enable characterization of both energetic and morphological effects of the catalyst compositions and a comparison between the resulting graphene films. Finally, thin film transfer techniques are developed to characterize post-growth metal catalysts with increased resolution and achievable sample sizes toward improving catalyst morphology understanding.

\subsubsection{Introduction}

PECVD synthesis of graphene was performed on primarily Ni bimetallic catalysts with varied concentrations of $\mathrm{Au}$ and $\mathrm{Cu}$ and compared to synthesis on trimetallic $\mathrm{NiAuCu}$ and pure $\mathrm{Ni}$ catalysts at $500^{\circ} \mathrm{C}$. While graphene films produced on pure $\mathrm{Ni}$ catalysts are multilayer, the addition of $1 \mathrm{wt} \% \mathrm{Au}$ or $2 \mathrm{wt} \% \mathrm{Cu}$ results in a significant reduction in multilayer portions of the graphene film. ${ }^{121,122}$ EBSD inspection of the catalyst films following growth reveals a reduction in grainsize with increasing $\mathrm{Au}$ content while grainsize remains comparable to pure Ni catalysts with small $\mathrm{Cu}$ additions. Synthesis performed on trimetallic catalysts with 98 wt $\%$ Ni 1 wt $\%$ Au and 1 wt $\%$ Cu results in primarily monolayer graphene formation and EBSD characterization reveals grainsizes comparable to pure Ni catalysts. To understand the varied graphene morphologies observed on each catalyst, first principle simulations were performed to identify differences in reaction energy for each step of acetylene decomposition on each catalyst. ${ }^{119}$ It is determined that, while both $\mathrm{Cu}$ and $\mathrm{Au}$ increase the energy required for acetylene decomposition when compared to $\mathrm{Ni}$, they exhibit varied modulations of each 
decomposition step. Simulation of decomposition on the trimetallic catalyst reveals a combination of effects observed with small $\mathrm{Au}$ and $\mathrm{Cu}$ additions and reveals a potential avenue for further control of graphene synthesis at reduced temperatures. While EBSD and simulation results reveal morphological and energetic data regarding the multimetal catalysts, identification of the location of added $\mathrm{Au}$ and $\mathrm{Cu}$ will be necessary to clarify the mechanism underlying graphene synthesis results. Toward achieving this level of resolution, a catalyst transfer technique is developed that enables millimeter scale characterization in a transmission electron microscope (TEM).

\subsubsection{Results and Discussion}

All graphene synthesis reported in this section is conducted at $500^{\circ} \mathrm{C}$ for 30 seconds with a $10 \mathrm{~W}$ ICP and $0.1 \mathrm{sccm}$ of $\mathrm{C}_{2} \mathrm{H}_{2}$ as the carbon precursor to enable characterization of catalyst compositional effects on achievable graphene quality under identical growth conditions. $50 \mathrm{~nm}$ metal catalysts were deposited onto $\mathrm{SiO}_{2}$ wafer supports with primarily Ni composition and Au contents of $1 \mathrm{wt} \%, 10 \mathrm{wt} \%$, and $15 \mathrm{wt} \%$, $\mathrm{Cu}$ contents of $1 \mathrm{wt} \%, 2 \mathrm{wt} \%$, and $5 \mathrm{wt} \%$, and a trimetallic composition of $\mathrm{Ni}(98 \mathrm{wt} \%)$ $\mathrm{Au}(1 \mathrm{wt} \%)$ and $\mathrm{Cu}(1 \mathrm{wt} \%)$. Graphene Synthesis was performed on each of these catalysts and compared to graphene grown on a $100 \mathrm{wt} \%$ Ni catalyst. Optimal graphene quality for each of the bimetallic catalysts, as determined by Raman characterization of average layer number and defect densities, was observed on $1 \mathrm{wt} \% \mathrm{Au}, 2 \mathrm{wt} \% \mathrm{Cu} .100$ $\mu \mathrm{m}^{2}$ Raman $\mathrm{I}_{2 \mathrm{D} / \mathrm{G}}$ maps of graphene grown on these compositions as well as the trimetallic and pure Ni catalysts is displayed in Figure 2.6. 
Comparison of graphene morphology from each catalyst reveals reduced multilayer formation with the addition of $\mathrm{Au}$ (Fig. 2.6: b) or $\mathrm{Cu}$ (Fig. 2.6: c) compared to the bulk Ni film (Fig. 2.6: a) with varied presentation of multilayer islands on each
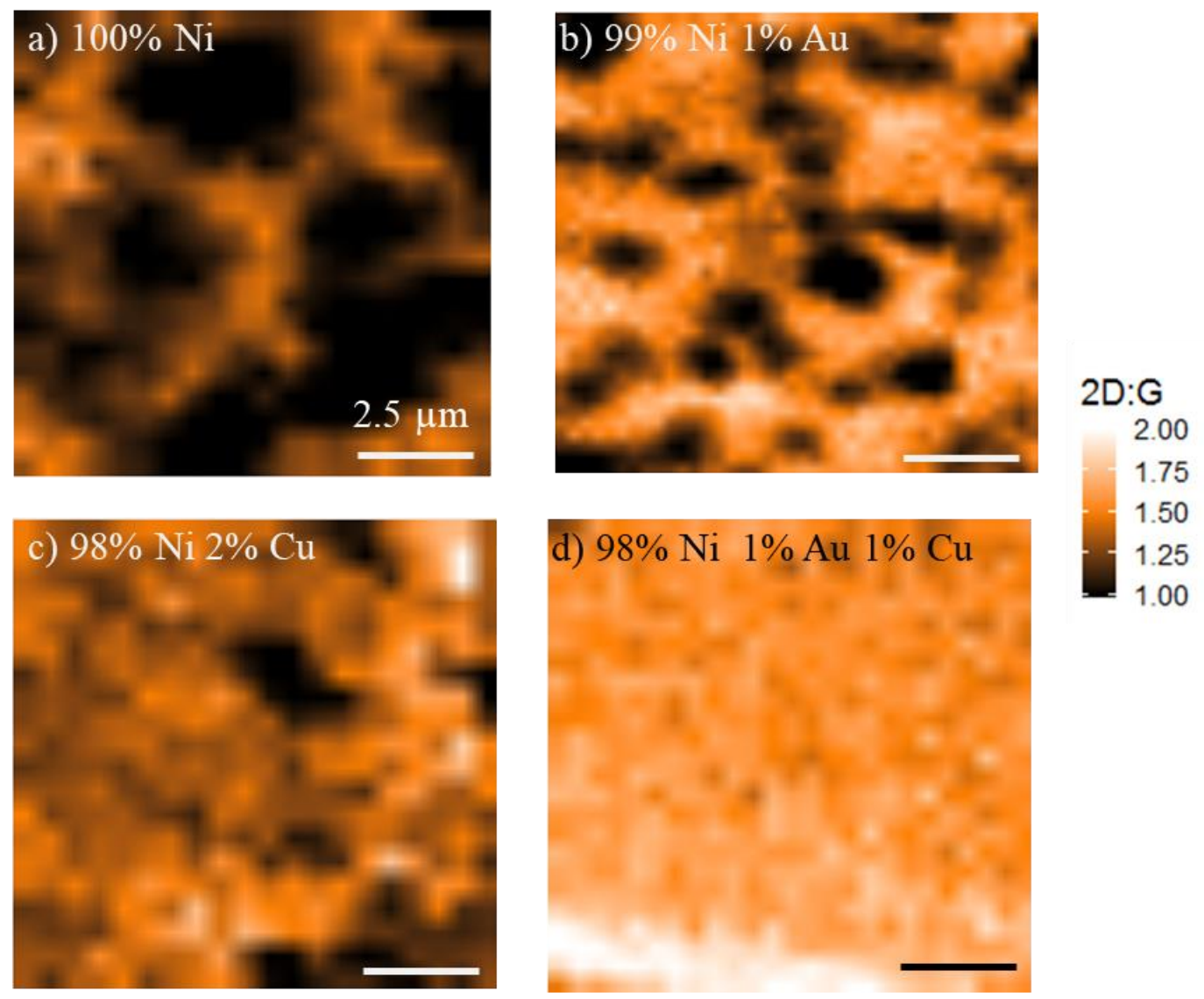

Figure 2.6: $100 \mu \mathrm{m}^{2}$ Raman $\mathrm{I}_{2 \mathrm{D} / \mathrm{G}}$ maps displaying $\mathrm{I}_{2 \mathrm{D} / \mathrm{G}}$ ratios of graphene films grown on metal thin film catalysts of a) pure Ni, b) 99 wt\% Ni 1 wt \% Au, c) 99 wt $\%$ Ni 2 wt\% Cu, and d) 99 wt $\%$ Ni 1 wt $\%$ Au 1 wt $\% \mathrm{Cu}$. Few-to-monolayer portions of the film are indicated by $\mathrm{I}_{2 \mathrm{D} / \mathrm{G}} \geq 1$ while multilayer portions of the films are black. A reduction in multilayer portions of the film are observed with the addition of $\mathrm{Au}$ or $\mathrm{Cu}$ as compared to the pure Ni catalyst and no multilayer portions are observed in the trimetallic catalyst. catalyst. The NiAu catalyst exhibits more multilayer islands (black spots) with few-tomonolayer graphene in-between while the $\mathrm{NiCu}$ catalyst shows primarily few layer graphene with fewer multilayer islands. Graphene grown on catalysts with increased concentrations of $\mathrm{Au}(10 \mathrm{wt} \%$ and $15 \mathrm{wt} \%)$ were both disconnected and increasingly multilayer while graphene from catalysts with both increased and decreased $\mathrm{Cu}$ content 
(1 wt $\%$ and $5 \mathrm{wt} \%$ ) showed increased multilayer, similar to the morphology of graphene on pure Ni. These results indicate that $\mathrm{Au}$ and $\mathrm{Cu}$ inclusion, while both capable of limiting graphene layer number when compare to pure Ni catalysts, display differing dependencies on composition percentage and result in different graphene morphologies under the same growth conditions. The increased number of multilayer islands observed on the NiAu catalyst, compared to the NiCu catalysts, may be a result of increased high reactivity portions of the catalyst, where nucleation and adsorption into the bulk occurs. In contrast, the increased average graphene layer number observed on the $\mathrm{NiCu}$ catalyst and reduced number of multilayer islands, as compared to the NiAu catalyst, may represent a more reactive surface overall that is also more uniform. Inspection of the trimetallic catalyst (Fig. 2.6: d) displays no presence of multilayer islands and demonstrates that a combination of low reactivity metals is capable of improving achievable graphene quality over the bimetallic counterparts.

While Raman characterization reveals varied graphene quality and uniformity on each catalyst composition, an understanding of the catalyst morphology is necessary to clarify the dominant effects in each case. To achieve this goal, EBSD inspection was performed following graphene synthesis. Band contrast and inverse pole figure $\mathrm{Z}$ (IPFz) images are displayed in Figure 2.7. Band contrast images are used as a representative measure of image quality, with increased image clarity signifying increased Kikuchi pattern intensity, while IPFz images represent the crystal lattice orientation of the catalyst grains in the z-direction, the catalyst surface in this case. 
Examination of the collected EBSD data reveals that the pure nickel catalyst (Fig. 2.7: a) exhibits primarily (111) and (001) crystal orientations while the NiAu catalyst (Fig. 2.7: b) displays an increased presence of (111) orientation with an accompanied

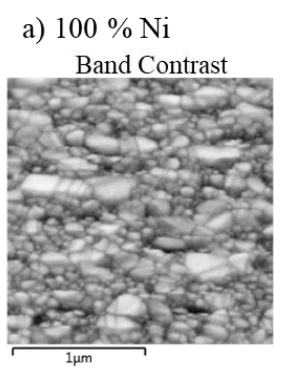

b) $99 \% \mathrm{Ni} 1 \% \mathrm{Au}$

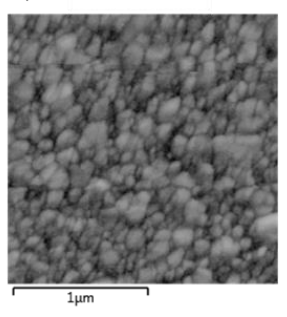

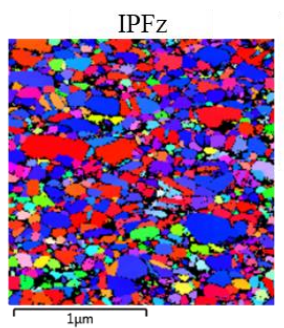
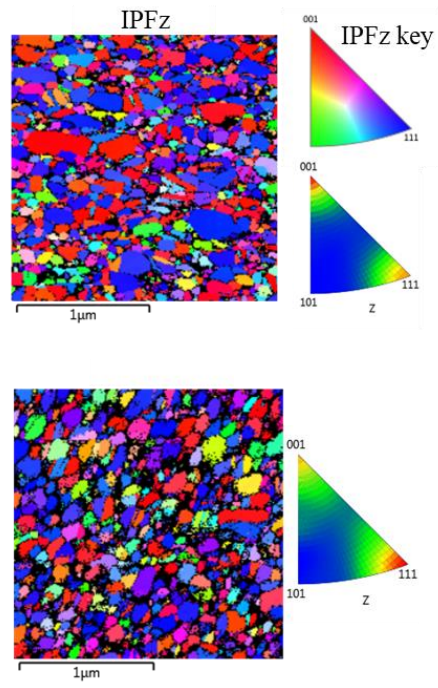

c) $98 \% \mathrm{Ni} 2 \% \mathrm{Cu}$
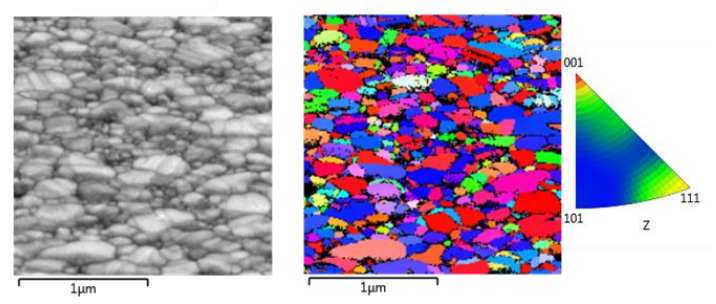

d) $98 \% \mathrm{Ni} 1 \% \mathrm{Au} 1 \% \mathrm{Cu}$
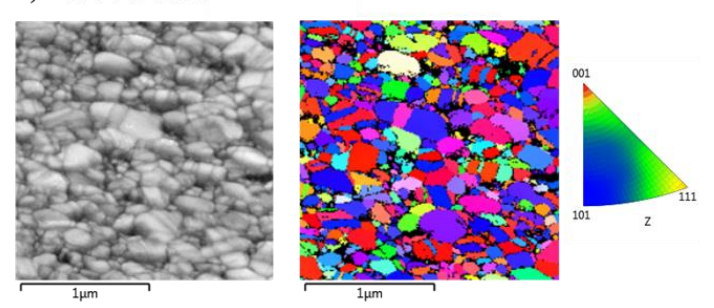

Figure 2.7: Band contrast and IPFz images from a) pure Ni, b) 99 wt $\%$ Ni 1 wt $\%$ Au, c) 99 wt $\%$ Ni 2 wt $\% \mathrm{Cu}$, and d) $99 \mathrm{wt} \% \mathrm{Ni} 1 \mathrm{wt} \% \mathrm{Au} 1 \mathrm{wt} \% \mathrm{Cu}$. Each IPFz map includes a IPFz heat map (to the right of each image) displaying concentrations of orientations detected, with red indicating increased concentrations of that orientation and the gradient from yellow to green to blue indicating reduced concentrations.

reduction in both identified portions of the map (black areas) and presence of large grains, when compared to the pure Ni Sample. Both the $\mathrm{NiCu}$ and NiAuCu catalysts (Fig. 2.7: $\mathrm{c}$ and d) display a reduced presence of (111) orientation and an increase in both grain size and presence of mixed phase grain orientations, between (001) and (111) in the z-direction, when compared to the pure Ni catalyst.

The reduced grainsize observed in the NiAu catalyst, which continues to decrease as additional Au is added, represents one potential cause of the increased number of multilayer islands observed in graphene grown on this catalyst when compared to the $\mathrm{NiCu}$ catalyst (Fig. 2.6: b and c). As grain boundaries represent high energy locations 
enabling both increased rates of hydrocarbon dehydrogenation and carbon absorption into the catalyst bulk, the increased grain boundary concentration may explain the observed increase in multilayer islands. This reduction in grainsize may originate from the lack of a shared phase between $\mathrm{Ni}$ and $\mathrm{Au}$ at the temperatures and compositions considered in this study and, under these conditions, Au has been proposed in literature to aggregate at Ni step edges. ${ }^{39,120}$ Additionally, the increased of presence of (111) crystal orientation may explain the observation of reduced layer numbers of graphene detected between multilayer islands on the NiAu catalyst (Fig. 2.6: b) as the (111) orientation has been described as less energetic for graphene synthesis than the $(001) .{ }^{123}$ In contrast, the shared phase between $\mathrm{Ni}$ and $\mathrm{Cu}$ at these temperatures and composition may enable alloy formation and result in an insignificant change to grainsize when compared to the pure $\mathrm{Ni}$ catalyst indicating an overall reduction in catalyst surface energy may be responsible for the graphene improvements observed on $\mathrm{NiCu}$ catalysts (Fig. 2.6: c) when compare to pure Ni catalysts (Fig. 2.6: a). ${ }^{124}$

A comparison of the EBSD data from the trimetallic catalyst (Fig. 2.7: d) to the $\mathrm{NiCu}$ catalyst (Fig. 2.7: c) reveals no apparent effect on the catalyst morphology originating from the Au inclusion. This lack of clear catalyst morphological differences between the $\mathrm{NiCu}$ and $\mathrm{NiAuCu}$ samples indicates that the significant improvements in graphene uniformity achievable on the trimetallic catalyst (Fig. 2.6: d) is not purely a result of grainsize or orientation variation. It is noted that the low temperature and short duration growth parameters results in small grainsizes which approach the limit of EBSD characterization, $\sim 20 \mathrm{~nm}$, and are below the spatial resolution limit of energy dispersive 
x-ray spectroscopy (EDX) in a SEM, on the order of $100 \mathrm{~nm}$ under ideal operating conditions. These challenges coupled with the inability to distinguish between $\mathrm{Ni}$ and $\mathrm{Cu}$ crystal phases in the collected EBSD spectrum limits identification of the precise location of $\mathrm{Au}$ and $\mathrm{Cu}$ in the multimetal catalysts with these techniques. ${ }^{125}$

While EBSD inspection provides an indication of morphological variations between catalyst compositions, further understanding of the improvements observed on the trimetallic catalyst requires characterization of the composition dependent energy differences for graphene synthesis on each sample. To achieve this goal, first principle simulations of acetylene decomposition were modeled on a $64 \mathrm{Ni}$ atom cell in the (100) orientation and compared to decomposition with varied concentrations of $\mathrm{Au}$ and $\mathrm{Cu}$ atoms substituted into the Ni lattice. The simulation consists of catalyst cells with 1, 2, 3, and $4 \mathrm{Cu}$ or $\mathrm{Au}$ atoms substituted in the Ni lattice at the surface location where $\mathrm{C}_{2} \mathrm{H}_{2}$ is decomposed and the results are compared to a pure Ni surface and a surface with $1 \mathrm{Au}$ and $1 \mathrm{Cu}$ atom as well as a surface with $2 \mathrm{Au}$ and $2 \mathrm{Cu}$ atoms. The decomposition is modeled as 2 dehydrogenation steps, resulting in the transformation of $\mathrm{C}_{2} \mathrm{H}_{2}$ to $\mathrm{C}_{2} \mathrm{H}+\mathrm{H}$ and finally to $\mathrm{C}_{2}+2 \mathrm{H}$, followed by decomposition of the carbon dimer, resulting in $2 \mathrm{C}+2 \mathrm{H}$ on the catalyst surface. The differences in reaction energy for each of these steps on the varied catalyst surfaces (Fig. 2.8) reveals that $\mathrm{Au}$ and $\mathrm{Cu}$ inclusions uniquely alter the ability of Ni to decompose an acetylene precursor. 
Examination of the simulation results indicates that $\mathrm{Cu}$ atom inclusions in the $\mathrm{Ni}$ catalyst (Fig. 2.8: a) decreases the energy required for dehydrogenation steps and increases the energy required for decomposition of the carbon dimer, compared to the pure Ni surface. Conversely, the addition of Au atoms (Fig. 2.8: b) increases the energy
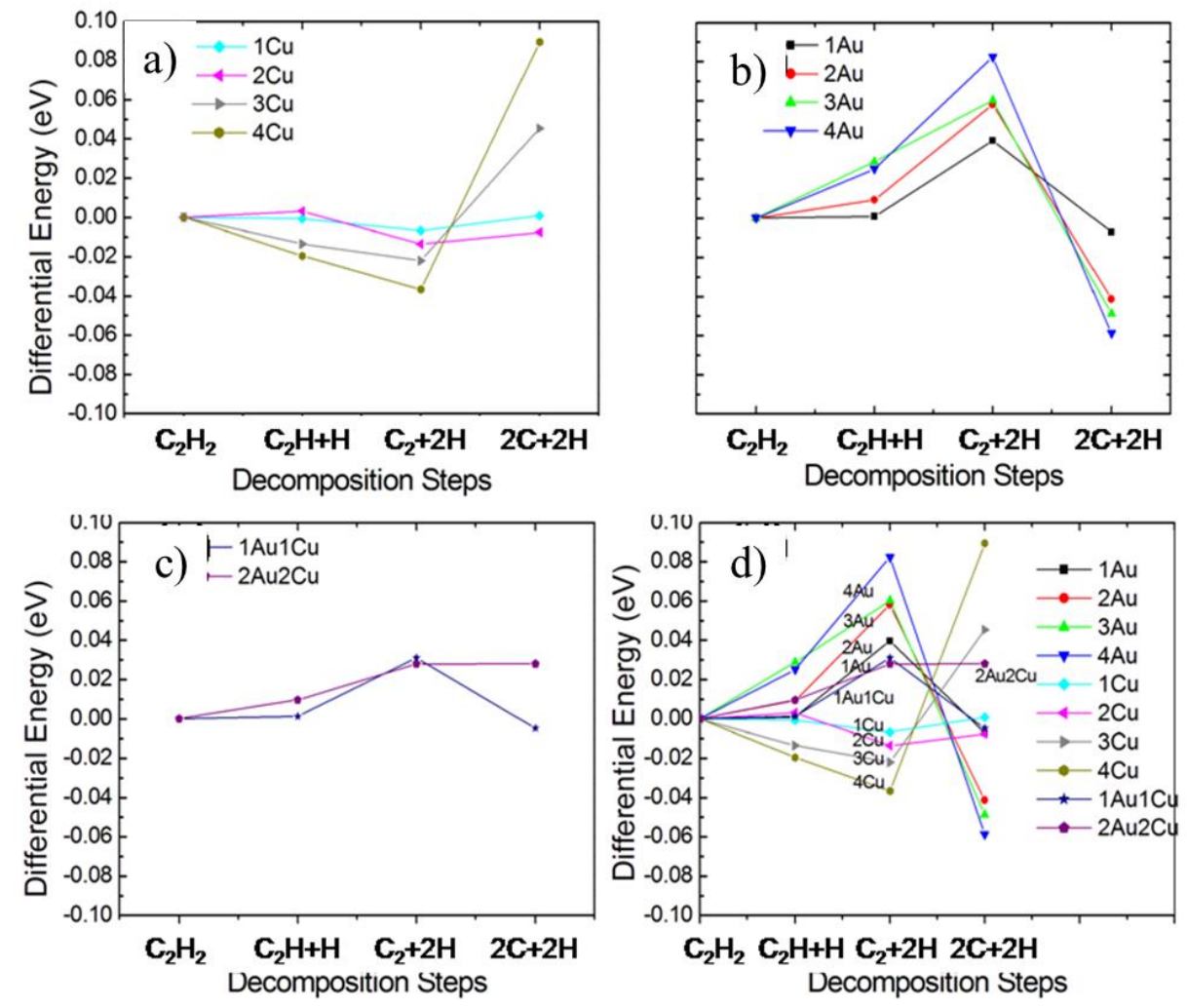

Figure 2.8: First principle simulation results for acetylene decomposition on primarily Ni catalyst surfaces with varied numbers of a) $\mathrm{Cu}$ atom substitutions b) $\mathrm{Au}$ atom substitutions and c) $\mathrm{Au}$ and $\mathrm{Cu}$ atom substitutions. d) Overlaid results from the 3 simulations. The data for each step is normalized to the energy calculated on a pure $\mathrm{Ni}$ catalyst and positive energies represent increased energy requirements for that decomposition step compared to on a pure Ni surface.

required for dehydrogenation and reduces the energy required for decomposition of the carbon dimer. The trimetallic catalyst surface (Fig. 2.8: c) with $2 \mathrm{Au}$ and $2 \mathrm{Cu}$ atoms substituted into the Ni lattice reveals an increase in energy requirements for both dehydrogenation and decomposition of the carbon dimer when compared to the pure $\mathrm{Ni}$ case. As multilayer formation is the primary obstacle to uniform graphene synthesis on 
Ni catalysts, reducing the rate of dehydrogenation and carbon decomposition is a promising strategy towards limiting catalyst saturation, due to reduced likelihood of carbon dimer absorption into the bulk, and reducing the growth rate, enabling enhanced control over the number of graphene layers produced. The reduction in catalyst reactivity for each decomposition step observed in the trimetallic catalyst provides a mechanism for the observed increase in uniformity and decrease in average layer number of graphene grown on this catalyst (Fig. 2.6: d). ${ }^{126}$

With EBSD revealing morphological differences and simulation indicating energetic modulation with varied catalyst composition, the utility of catalyst content design as a pathway for uniform graphene synthesis at reduced temperatures has been shown. However, while first principle simulations reveal trends with changing metal content for the decomposition of acetylene, the reaction environment during PECVD synthesis has been shown to contain numerous hydrocarbon species with varied ionization states. ${ }^{111,127}$ Additionally, EBSD has shown the growth catalyst to be more disordered than the single crystal orientation with individual atom substitutions used in these simulations. To further optimize catalysts toward continued graphene synthesis temperature reduction, identification of $\mathrm{Au}$ and $\mathrm{Cu}$ locations in the bulk Ni catalyst will be necessary.

To increase the achievable resolution for catalyst inspection, an electrochemical transfer method for metal thin films was developed enabling millimeter sized portions of the film to be examined via transmission electron microscopy (TEM). 

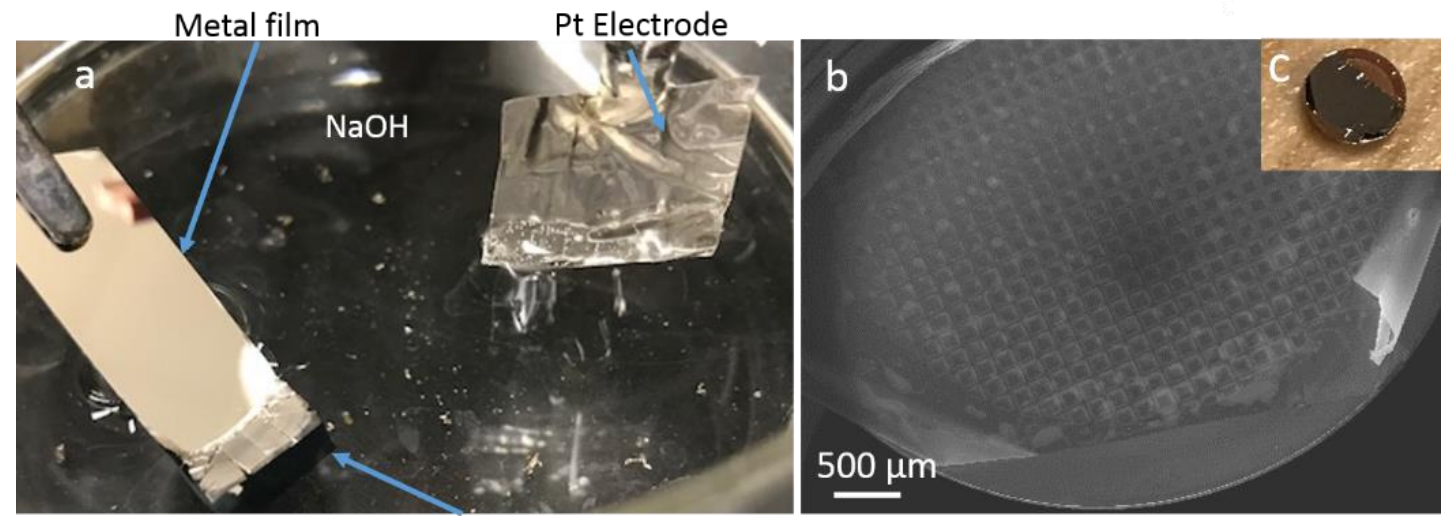

$\mathrm{SiO}$, support

Figure 2.9: a) Image of electrochemical transfer mechanism displaying the metal thin film delaminating from the $\mathrm{SiO}_{2}$ support wafer in $\mathrm{NaOH}$ electrolyte. b) SEM image of metal thin film on TEM support grid with c) optical image inset.

We observed that thin films with weak adhesion to $\mathrm{SiO}_{2}$ would delaminate during the electrochemical graphene transfer process. This initially disruptive phenomenon was optimized to enable large scale transfer of metal thin film catalyst from $\mathrm{SiO}_{2}$. The $\mathrm{SiO}_{2}$ supported metal thin film is placed in a minimally reactive electrolyte, $\mathrm{NaOH}$, where it acts as an anode to a platinum cathode (Fig. 2.9: a). Following initial delamination of the edge of the film, it is lowered into the electrolyte at an angle such that $\mathrm{O}_{2}$ bubble generation occurs primarily at the $\mathrm{SiO}_{2} /$ metal interface. The floating film is then washed and transferred to a TEM grid (Fig. 2.9: c) and imaged in an SEM Transmission Kikuchi Diffraction (TKD) holder (Fig. 2.9: b).

A TKD IPFz map of the transferred thin film (Fig. 2.10: a) was compared to an EBSD IPFz map (Fig. 2.10: d) of the catalyst on $\mathrm{SiO}_{2}$ support to demonstrate the order of magnitude improvement in spatial resolution achievable with the TKD process. ${ }^{128}$ Maps were taken with $10 \mathrm{~nm}$ step sizes on catalyst thin films from a single graphene synthesis and no data processing was performed. 

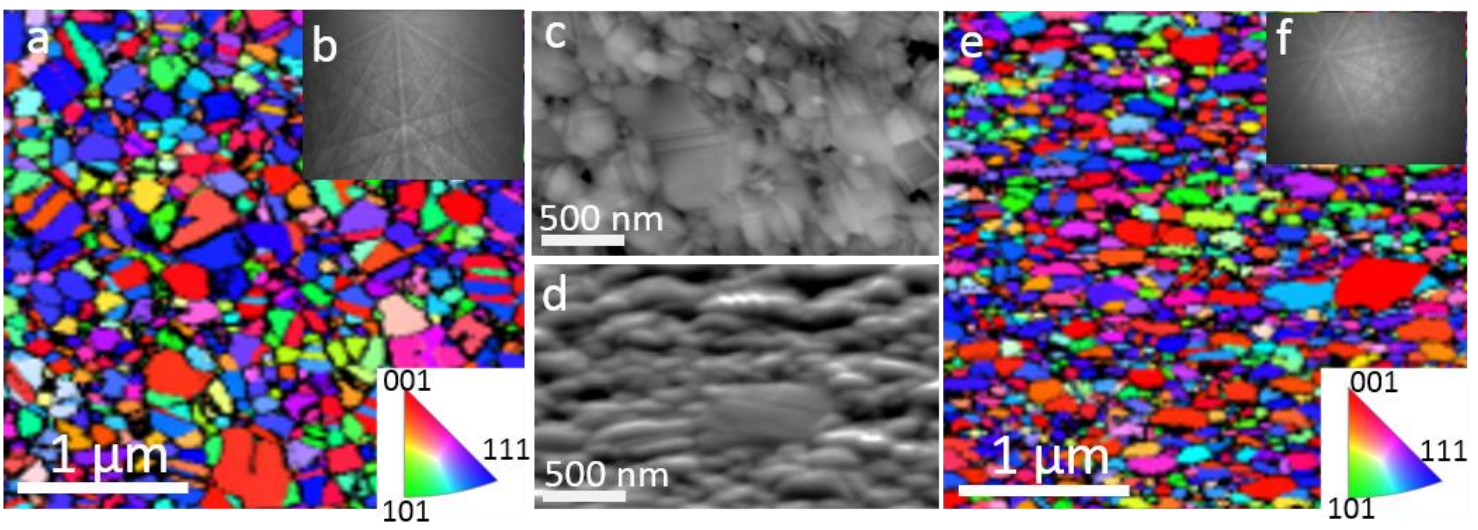

Figure 2.10: a) Unprocessed TKD IPF Z map with b) inset sample Kikuchi pattern and c) FSD image of transferred metal thin film d) FSD image and e) Unprocessed IPF Z EBSD map with f) inset sample Kikuchi pattern of metal thin film on $\mathrm{SiO}_{2}$ support before transfer.

Maps and Forward Scatter Detector (FSD) images were taken with the transferred film at $-20^{\circ}$ tilt with respect to the incident beam (Fig. 2.10: c) and the as-synthesized film at $70^{\circ}$ tilt (Fig. 2.10: d). The increase in achievable spatial resolution is most prominently displayed in the presence of twinning and clear grain boundaries in the TKD map while similar features in the EBSD map are often unresolved. This improvement is associated with increased Kikuchi pattern strength (Fig. 2.10: b, f) resulting from a reduced interaction volume in the transferred film compared to the $\mathrm{SiO}_{2}$ supported film and the reduced sensitivity to surface morphology during TKD acquisition.

TEM inspection of the transferred thin film revealed a suspended non-metal thin film near edges of a hole in the catalyst (Fig. 2.11: a). Much of the suspended film was damaged and curled (Fig. 2.11: b), however, examining near the interface and focusing on the plane of the metal enabled simultaneous observation of lattice fringes for both the catalyst and the non-metal film (Fig. 2.11: c). 


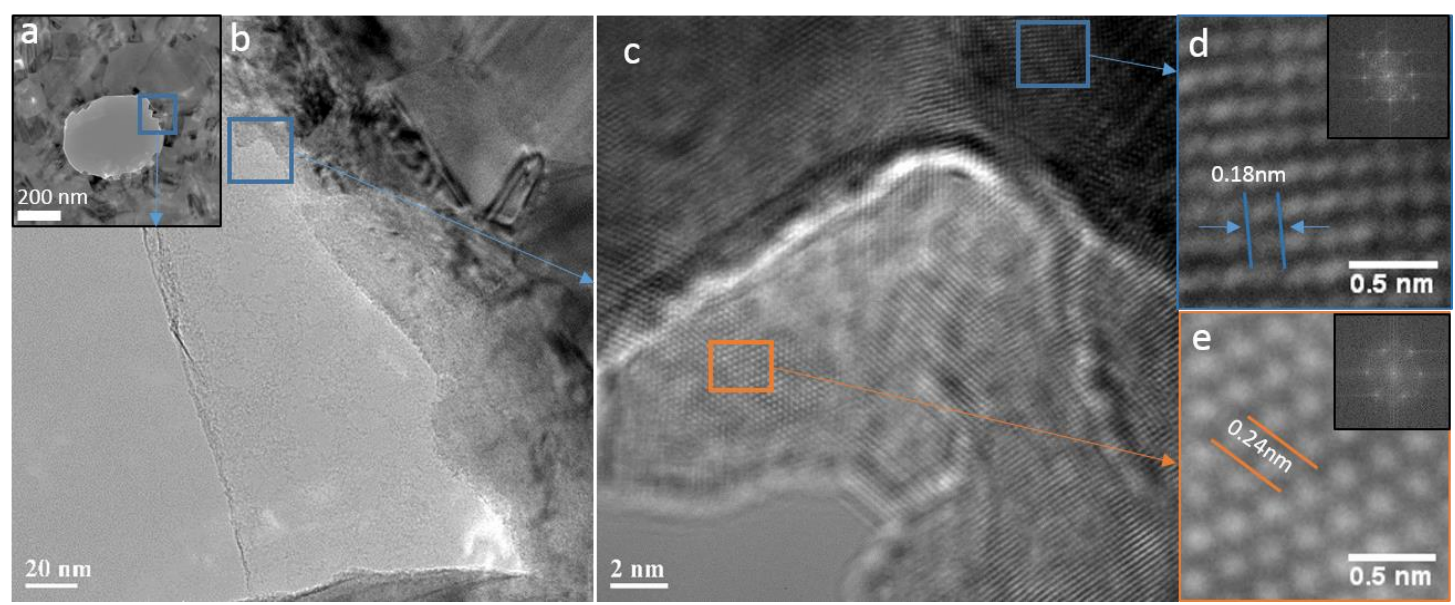

Figure 2.11: a) Bright field TEM image of transferred metal thin film. b) Magnified section of the blue box in a) with both metal and graphene visible. c) Magnified image of the blue box in b) with both d) Ni lattice fringes and e) graphene lattice fringes visible.

Measurements and FFT of these regions of interest reveal characteristic lattice spacing of Ni, (Fig. 2.11: d), and graphene (Fig. 2.11: e) indicating that the electrochemical transfer method is primarily active at the $\mathrm{SiO}_{2} /$ metal interface, leaving the graphene on the top surface largely intact. This transfer method represents an avenue for further multimetal catalyst characterization toward identifying the location of small $\mathrm{Au}$ and $\mathrm{Cu}$ components of primarily Ni catalysts that will be necessary to describe the underlying mechanisms for graphene improvements observed on trimetallic catalysts. ${ }^{118}$

\subsubsection{Summary}

We have demonstrated the first reported PECVD synthesis of graphene on $\mathrm{NiAuCu}$ thin film catalysts which show improvements in both graphene layer number and uniformity control at $500^{\circ} \mathrm{C}$. Graphene synthesized on these trimetallic catalysts displays primarily few-to-monolayer coverage with no observable multilayer islands that occur on bimetallic $\mathrm{NiAu}$ or $\mathrm{NiCu}$ catalysts under identical growth conditions. EBSD characterization of the catalyst films reveals morphology differences between $\mathrm{NiAu}$ and 
$\mathrm{NiCu}$ samples indicating that grainsize and orientation play a role in achievable graphene quality. First principle simulations of acetylene decomposition on these varied catalyst compositions shows $\mathrm{Au}$ inclusion reduces dehydrogenation rates while $\mathrm{Cu}$ inclusion reduces decomposition rates of the carbon dimer and that the amount of each added metal can be adjusted to modulate graphene growth rates. To enable the identification of the precise locations of $\mathrm{Au}$ and $\mathrm{Cu}$ catalyst constituents in the thin film, a transfer technique for large sample area TEM inspection and increased EBSD resolution is demonstrated. These findings represent additional avenues for continued graphene synthesis optimization and reduction of synthesis temperature and catalyst thickness requirements toward direct graphene inclusion in CMOS applications.

\subsubsection{Experimental Methods}

Graphene Synthesis and Catalyst Deposition

Graphene was synthesized in the custom PECVD system described in section 2.2.1. Catalysts were deposited via magnetron sputtering for $\mathrm{Ni}$ and $\mathrm{Cu}$ and electron beam evaporation for NiAu in a Kurt J. Lesker AXXISS PVD system. $50 \mathrm{~nm}$ catalysts were deposited on $\mathrm{Si} / \mathrm{SiO}_{2}$ wafers at $1 \mathrm{wt} \%, 10 \mathrm{wt} \%$ and $15 \mathrm{wt} \% \mathrm{Au}, 1 \mathrm{wt} \%, 2 \mathrm{wt} \%$, and 3 wt $\% \mathrm{Cu}$, or $1 \mathrm{wt} \%$ Au together with $1 \mathrm{wt} \% \mathrm{Cu}$, with the remainder of the catalyst as $\mathrm{Ni}$. Graphene synthesis was performed within the PECVD at a reaction temperature of $500^{\circ} \mathrm{C}$ for 30s with a $10 \mathrm{~W}$ inductively coupled plasma and $0.1 \mathrm{sccm}$ flow rate of $\mathrm{C}_{2} \mathrm{H}_{2}$ at a chamber pressure of $4 \times 10^{-6}$ torr.

Sample Characterization and Modeling 
After the synthesis, the catalyst was etched away and graphene was transferred to fresh $\mathrm{SiO}_{2}$ substrates for Raman inspection in a Horiba Jobin Yvon $\mathrm{Hr} 800$ spectrometer with a 532nm excitation laser. EBSD inspection was performed in a Zeiss Sigma SEM with Oxford EBSD and EDX detectors, while TEM inspection was performed in a FEI Tecnai F-20 system. First principle simulation was carried with ABINIT software and EBSD map characterization was performed in Oxford's AZtec software package.

\subsection{From CMOS to beyond-CMOS applications}

This chapter has demonstrated the development of techniques necessary for graphene synthesis temperature and catalyst thickness reduction through plasma manipulation and catalyst engineering. These reductions are needed to realize CMOS incorporation of graphene where target applications require nonplanar coverage and the magnitude of improvement scales with a reduction of the total films thickness. Carbon precursor control during synthesis coupled with trimetallic catalyst design and fabrication represent fruitful paths for continued research toward graphene inclusion in CMOS applications. In the following section, graphene as a component of beyond CMOS computing applications will be detailed, where system design and operational phenomena represent roadblocks to incorporation rather than stringent processing and incorporation requirements. To demonstrate scalability of graphene inclusion, the following section will utilize commercially available graphene and device fabrication amenable to semiconductor industry inclusion to examine spin transport phenomena. 


\section{3: Graphene Spin Transport Characterization}

Graphene represents an attractive spin transport channel candidate for spintronic devices due to the theoretically predicted long spin transport distances and spin lifetimes. However, experimentally realized values for these parameters are orders of magnitude smaller those theoretically predicted and numerous mechanisms have been proposed to explain this discrepancy, including magnetic contact induced spin relaxation, tunnel barrier inhomogeneity, and a lack of clarity associated with the dominant spin relaxation mechanism in graphene channels. ${ }^{129}$ Additionally, the majority of characterization has occurred with a NLSV device which enables generation of pure spin currents for determination of spin transport and spin lifetimes but does not enable robust characterization of proposed spin relaxation phenomena associated with device components. While $\mathrm{TB}, \mathrm{h}-\mathrm{BN}$ encapsulation, and external field modifications to the standard NLSV have enabled detection of increased spin signals, proposed spintronic devices will necessitate device components not represented in the standard NLSV, including additional FM contacts for logic operations and angled channels for interconnected device configurations. Finally, few studies have reported on wafer scale graphene based spin valve fabrication through processes amenable to inclusion in the current semiconductor industry. In this report, we design a new type of graphene spin valve, the hybrid drift diffusion spin valve (HDDSV), fabricate large arrays of these devices through industry standard fabrication techniques, and identify unique phenomena arising from the novel device design. 


\subsection{Introduction}

In this study we present a new device configuration for graphene spin transport characterization to identify device component effects not represented in the standard NLSV. The HDDSV device includes two locations for nonlocal spin detection, one in the standard NLSV location and one located along a graphene channel originating at an angle from the injection leg of a standard NLSV. As this device architecture represents a new configuration, arrays consisting of 120 devices, 82 HDDSV, 30 NLSV, and 8 graphene characterization devices, are designed to enable characterization of FM contact spacing, channel width, channel angle, and channel length effects on spin transport capabilities. Fabrication techniques for 4-inch wafer scale production of 36 arrays, representing a total of 4320 devices, are developed to enable demonstration of industry applicable fabrication techniques for graphene based spin devices. Oxidation procedures for $\mathrm{TiO}_{2} \mathrm{~TB}$ fabrication are developed and characterized to determine spin signal and device noise magnitudes with varied FM injector and magnetic contact resistances. Spin signals $\left(\Delta \mathrm{R}_{\mathrm{NL}}\right)$ of up to $2.6 \Omega$ are detected at the lowest performed oxidation condition, 1 torr, and background noise is observed to decrease as metal contact resistance is minimized through altered deposition techniques. NLSV legs of the devices are compared before and after an oxidation procedure to determine environmental effects with an average spin signal increase of $193 \%$ observed, up to $7.6 \Omega$, accompanied with changing FM contact magnetic switch profiles. Characterization of HDDSV devices reveal increased stability of antiparallel states on the NLSV leg with the inclusion of a second FM detection magnet, representing a potential path toward increased device state targeting, in either the parallel or antiparallel orientation. Finally, characterization of HDDSV signals in the 
added channel yield spin signals reaching $865 \Omega$, with signal detection in both nonlocal and local configurations, representing a path toward reduced device complexity and increased spin signal generation. Characterization of 5 arrays of HDDSV and NLSV devices has revealed spin transport phenomena not readily detected in standard device configurations and identified areas in both fabrication and devices design for further improvement and investigation to enable graphene inclusion in spintronic applications.

\subsection{Hybrid NLSV Device design}

This research reports the design and fabrication of a novel hybrid drift diffusion spin valve (HDDSV) (Fig. 3.1) device to reveal graphene spin transport phenomena that are not detectable by commonly used non-local spin valves (NLSV). The device is designed to characterize FM contact effects while utilizing charge carrier drift and

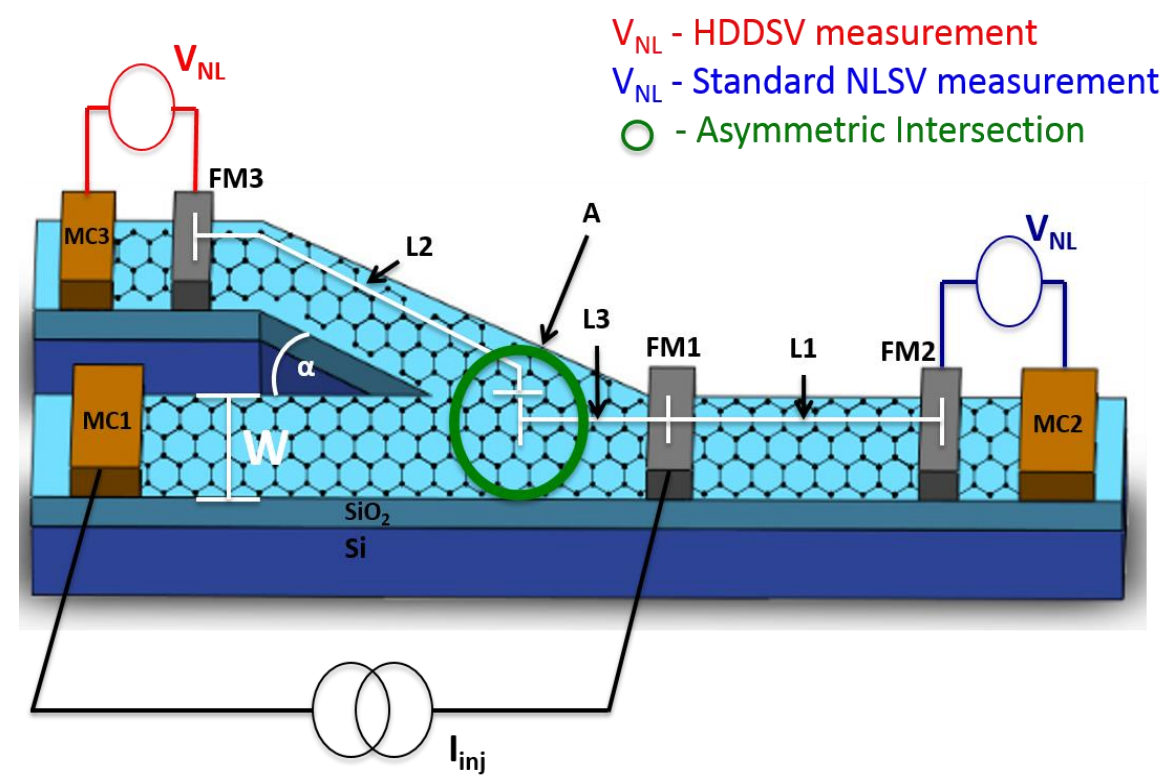

Figure 3.1: The schematic of proposed graphene HDDSV consists of three metal contacts (MC1-3), three ferromagnetic contacts (FM1-3), and a split graphene transport channel. Spin polarized injection occurs between FM1 and MC1. The standard NLSV nonlocal voltage measurements can be taken between FM2 and MC2, while a hybrid spin drift and diffusion measurement can be measured between FM3 and MC3.

momentum to enhance spin diffusion lengths and achievable spin signal. The proposed 
capabilities arise through detecting nonlocal signals originating from a spin accumulation of spin polarized charge carriers, which occurs away from the influence of the ferromagnetic (FM) injection contact. The structural configuration of proposed HDDSV is modeled after the standard NLSV, with an additional graphene channel (as depicted in Fig. 3.1). This device enables direct comparison between graphene spin transport parameters collected in a standard NLSV and in an adjacent graphene channel.

The aim of this modified design is to enable determination of the fundamental effects of FM/TB contacts and charge carrier drift in relation to achievable spin signals in graphene. It can be operated as a standard NLSV or in a hybrid drift-diffusion mode. This enables comparisons between diffusion from spin accumulation at the FM injector in standard NLSV mode or at the asymmetric intersection (as marked by the green circle A in Fig. 3.1) in a hybrid drift and diffusion mode, providing information relevant to FM/TB/Graphene interface effects on spin transport. It provides flexibility for manipulating device dimensions to identify effects on graphene spin transport parameters. In particular, variation in graphene channel lengths (L1/L2/L3), channel widths (W), the angle ( $\alpha$ ) between the spin polarized current channel (between FM1 and MC1), and the additional nonlocal spin diffusion channel (L2) will enable characterization of configuration-dependent spin signals, injector current dependencies, and field enhanced spin diffusion. As local magnetic fields present near the injector and detector FM contacts have been proposed to contribute to premature spin relaxation, ${ }^{130}$ separation of the asymmetric intersection from the injection FM is performed to characterize this effect while variation of the intersection angle $(\alpha)$ is aimed at 
determining transfer of momentum from the injected electrons to those diffusing down the additional channel. Additionally, the inclusion of a second detection FM contact (FM 3) may reveal additional magnetic influences on both spin transport and the behavior of FM switching fields to identify phenomena present in device configurations displaying increased complexity required for the realization of spintronic applications.

Utilizing device fabrication and processing techniques with industry applicable and scalable procedures and materials, HDDSV devices are designed with multilayer $\mathrm{Ti} / \mathrm{Au}$ as metal electrodes, $\mathrm{Co}$ as $\mathrm{FM}$ electrodes, $\mathrm{TiO}_{2}$ as tunnel barriers (TBs), and $\mathrm{SiO}_{2}$ as the substrate. The graphene used for spin transport channels is sourced commercially (Graphene Square) on 4 inch $\mathrm{SiO}_{2}$ wafers to demonstrate scalable fabrication techniques on mass produced and widely available graphene. Device patterning is performed through electron beam lithography (EBL) while metal (Ti/Au), $\mathrm{FM}(\mathrm{Co})$, and $\mathrm{TB}\left(\mathrm{TiO}_{2}\right)$ device components are deposited by electron beam evaporation (EBE) with in situ oxidation of Ti performed on TBs to form an oxide. Though reported results on devices with h-BN encapsulation, MBE deposited TBs, and exfoliated graphene display increased spin transport capabilities, the fabrication and materials selected in the design of these devices is aimed at developing scalable and high throughput techniques. ${ }^{131}$

While the design of HDDSV devices is targeted to enable characterization of electron diffusion and drift interactions as well as FM contact influences, the device architecture contains components that have not been characterized previously and 
necessitates numerous control devices and varied devices dimensions. To achieve a range of device configurations, EBL patterned devices consist of channel lengths of $1.5 \mu \mathrm{m}$ and $3 \mu \mathrm{m}$ (Fig. 3.2: c and Fig. 3.2: d L1+L2+L3), channel widths of $400 \mathrm{~nm}$ and $800 \mathrm{~nm}$ (Fig. 3.2: c: W), and an asymmetric channel angles of $45^{\circ}$ (Fig. 3.2: c: $\alpha$ ) and $90^{\circ}$ (Fig. 3.2: d:

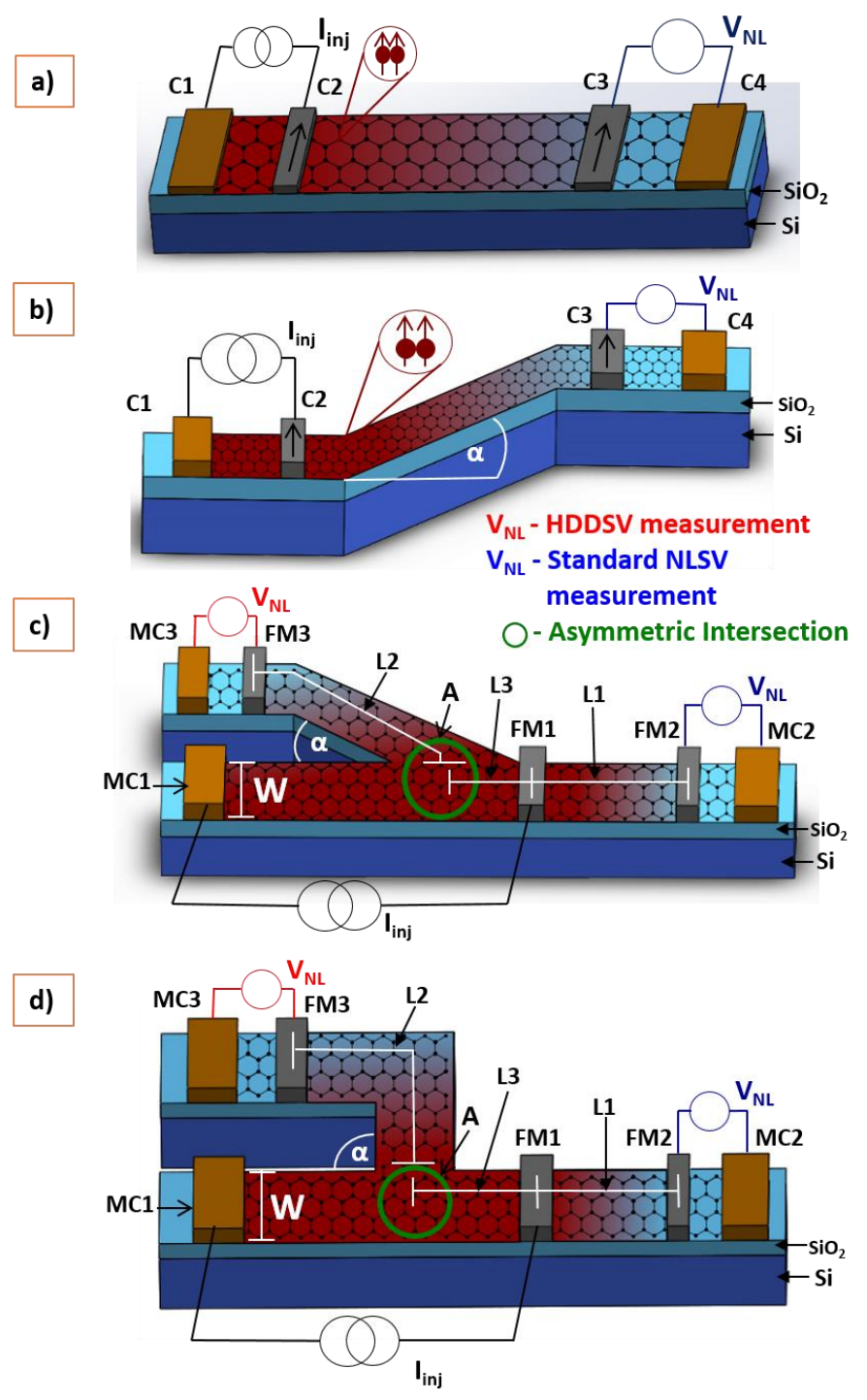

Figure 3.2: a) Standard NLSV geometry b) Control device for angled channel effect on NLSV parameters. c) Representative device configuration for FM contacts effects with spin accumulation at green circle in HDDSV operation mode. d) Representative device configuration for field and momentum effects in which the angle between diffusion and drift channels are varied. 
a). Additionally, control devices (Fig. 3.2: a and b) will be included in the array. In Figure $3.2 \mathrm{~b}$, a channel bend angle $(\alpha)$ between $\mathrm{C} 2$ and $\mathrm{C} 3$ is created, in contrast to the straight channel of a standard NLSV (Fig. 3.2: a). Characterizing both the standard NLSV and angled NLSV control devices will reveal spin transport phenomena resulting from an angled channel, in which diffusing electrons are traveling with a component parallel to both the applied magnetic field and the alignment of the injection and detection FM contacts, compared to a straight channel, in which a perpendicular orientation is maintained. For all other HDDSV devices, a second bend is included before MC3/FM3 to enable alignment of all FM contacts perpendicular to the in-plane magnetic field used to manipulate FM polarization during spin parameter measurements. Spin parameters collected from these devices is targeted towards characterization of device configuration, channel width, and FM contact effects.

HDDSV device arrays (Fig. 3.3) were designed that contain 120 individual devices with varying graphene channel widths, lengths between injection and detection magnetic contacts, asymmetric drift/diffusion channel angles, and control devices to characterize targeted graphene quality and device component effects on achievable graphene spin transport lengths. The design enables multi-device characterization over large graphene areas in an effort to isolate graphene quality effects from device configurational effects with sufficient numbers of devices to identify new phenomena and 
enable statistical treatment of results.

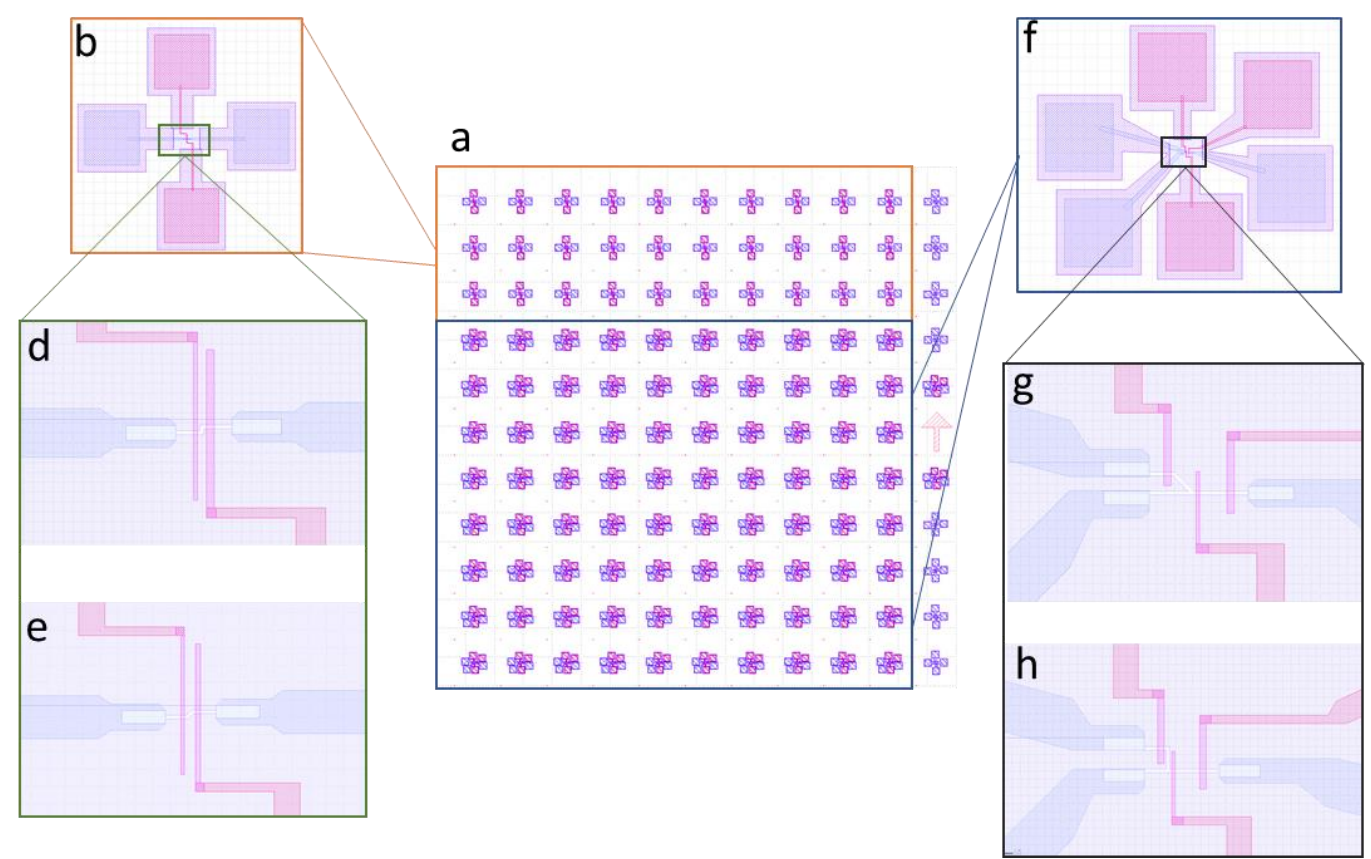

Figure 3.3: a) GDSII file for a 120 device array containing 82 HDDSV devices, b) 30 control devices and 8 test devices. The 30 control devices include straight, d) 45-degree, and e) 90-degree asymmetric diffusion transport channels with varying nonlocal transport distances, injection and detection magnetic contact dimensions, and graphene transport channel widths. f) The 82 HDDSV devices include both g) 45-degree and h) 90-degree asymmetric diffusion transport channels with varying nonlocal transport distances, injection and detection magnetic contact dimensions, and graphene transport channel widths.

\section{3: Wafer Scale Device Fabrication}

Fabrication of 36 device arrays detailed in the previous section was performed on graphene purchased from Graphene Square on a 4 inch $\mathrm{SiO}_{2}$ wafer (Fig. 3.4: a) through

$\mathrm{EBE}$ and EBL processes to demonstrate the viability of scalable and high throughput processes for the production of graphene based spin devices. The fabrication process involves 4 EBL patterning steps (alignment marks, graphene channel and contact locations, metal contacts, and ferromagnetic contacts), graphene removal via oxygen plasma etching to define the graphene transport channel and isolate contacts, and 3 metal depositions (alignment marks, metal contacts and tunnel barriers/ferromagnetic contacts). 
Metal contacts were deposited via EBE of $5 \mathrm{~nm} \mathrm{Ti}$, to improve adhesion to the graphene channel, followed by EBE of $50 \mathrm{~nm}$ of $\mathrm{Au}$, a malleable and inert contact material to enable repeated device characterization. Following deposition of the metal contacts (Fig. 3.4: b,c) 3 arrays were characterized for connection between metal contacts and 232 devices of the 360 inspected were observed to have connected graphene channels

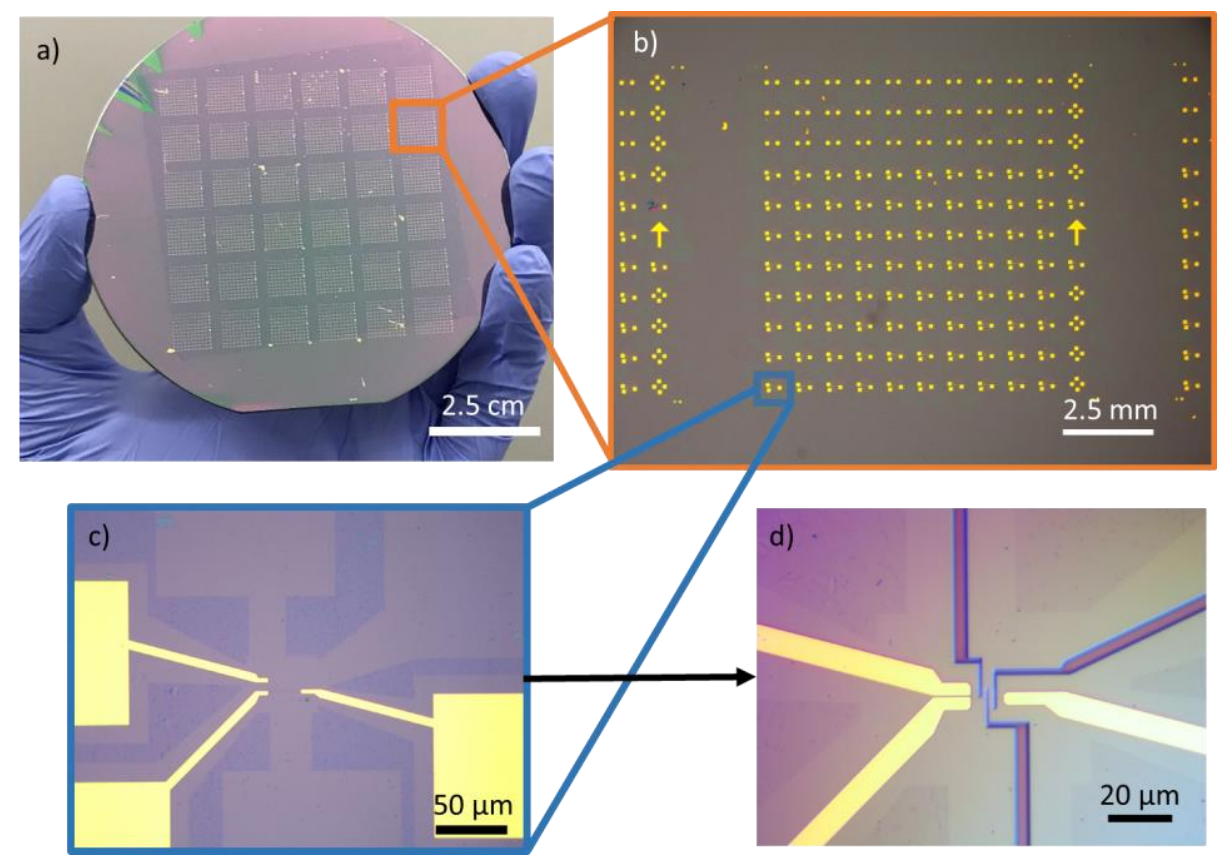

Figure 3.4: a) 4 inch wafer with 36 HDDSV arrays each containing 120 graphene devices. b) Optical image of individual HDDSV array with c) individual device with metal contacts present and d) the same device following a forming gas anneal procedure and EBL patterning of the FM contacts.

representing $64.4 \%$ active devices. When only devices with $800 \mathrm{~nm}$ channel widths are considered, 171 devices of the 198 characterized were determined to have intact graphene channels representing a yield of $86.3 \%$, representing yield scaling with channel width and pointing to channel definition through etching as a significant failure point. These results demonstrate the feasibility of wafer scale graphene device fabrication through techniques commonplace in the semiconductor industry with commercially available graphene. 
Following initial characterization of graphene arrays, a graphene surface cleaning process was performed to remove residual contamination from the previous lithography processing steps before patterning of the FM contacts (Fig. 3.4: d). Achievable spin

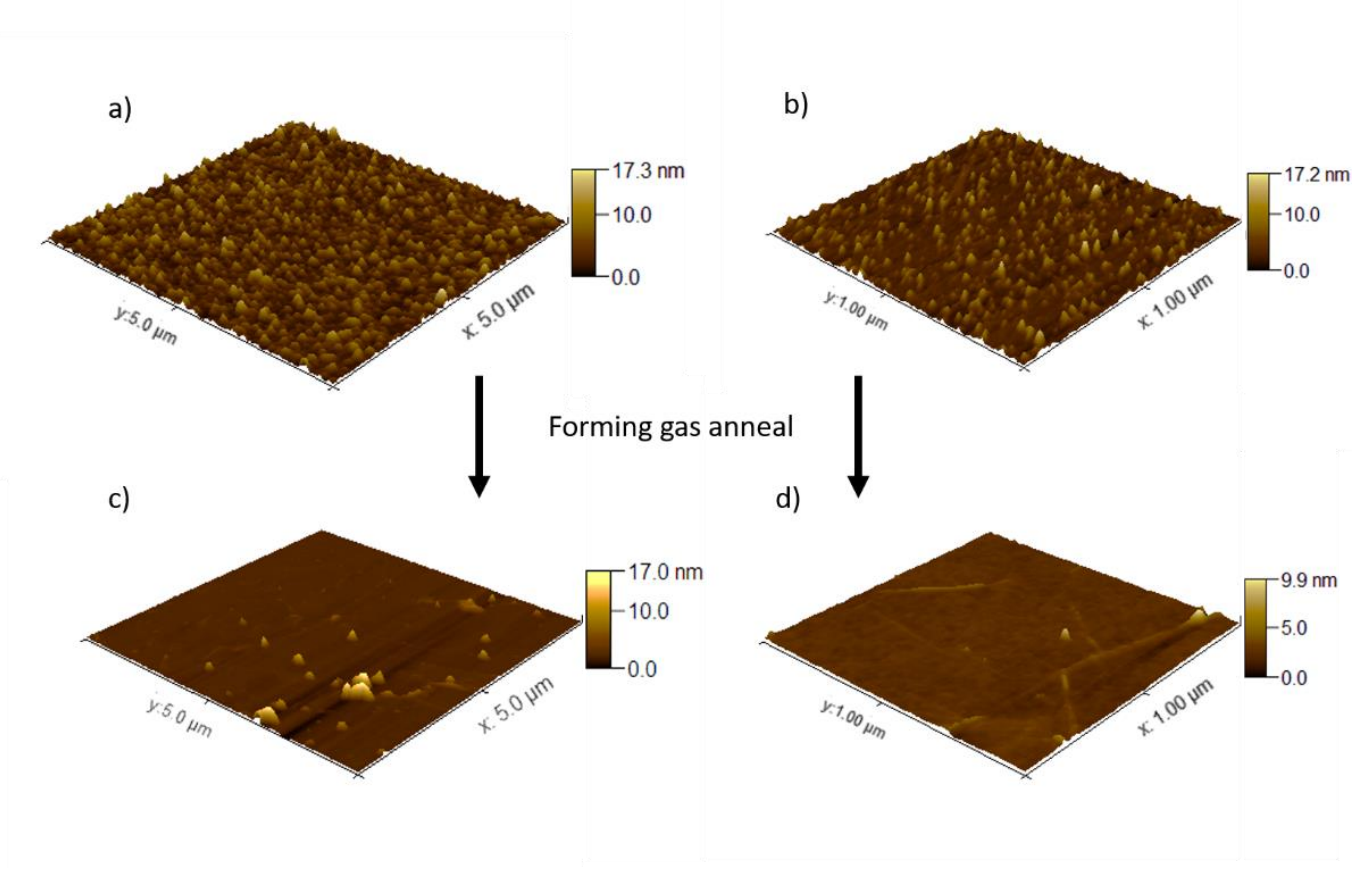

Figure 3.5: a,b) Atomic force microscopy images displaying graphene surface contamination from residual lithography mask polymers. c,d) Graphene surface following forming gas anneal indicating a significant reduction in contaminates present at the graphene surface.

signals are highly influenced by the interface between the TB and graphene channel. ${ }^{132,133}$ This $\mathrm{TB}$ /graphene interface determines the efficiency of spin injection into the channel and the integrity of TB influences the degree of spin reabsorption into the FM contact. ${ }^{134,135}$ During EBL processing, polymer resist masks are utilized to define both the graphene channel and metal contact locations, resulting in a residual polymer coating following removal of the resist. Atomic force microscopy (AFM) inspection of graphene surface following removal of the metal contact resist reveals graphene RMS surface roughness of $1.72 \mathrm{~nm}$ (Fig. 3.5: a,b). As the $\mathrm{TiO}_{2}$ tunnel barrier used in this study is $\sim 1$ 
$\mathrm{nm}$ in thickness, this level of surface roughness not only represents a significant concentration of material in between the graphene channel and the TB but a rough surface profile leading to increased likelihood of crack and pinhole formation in the $\mathrm{TiO}_{2}$. This residue is removed though annealing of the devices at $300^{\circ} \mathrm{C}$ in a forming gas $(95 \%$ $\mathrm{N}_{2} / 5 \% \mathrm{H}_{2}$ ) atmosphere for 2 hours which reduces RMS surface roughness to $200 \mathrm{pm}$, without degrading the graphene quality (Fig. 3.5: c, d).

With TB/FM contacts pattered via EBL with $200 \mathrm{~nm}$ injector widths and $400 \mathrm{~nm}$ detector widths, the wafer is separated into individual arrays to characterize various TB oxidation conditions to optimize spin signal to noise ratios. Immediately prior to $\mathrm{TB}$ deposition, the device array is heated to $120^{\circ} \mathrm{C}$ for 2 minutes at $1.4 \times 10^{-7}$ torr in the physical vapor deposition (PVD) chamber to clean the exposed graphene surface at the bottom of the patterned trenches. While an additional forming gas anneal procedure at this step would be preferred, the lithography resist is not capable of maintaining device dimensions at the required temperatures $\left(300^{\circ} \mathrm{C}\right)$ for the forming gas procedure. TBs were deposited in a 2-step process where $0.4 \AA$ of Ti is deposited via EBE followed by in situ oxidation at the target pressure for 10 minutes. This process is then repeated, followed by EBE of $25 \mathrm{~nm}$ of Co to complete the TB/FM contact. Oxidations on individual device arrays were performed at 75 torr, 10 torr, 1 torr, and 1 torr with the array allowed to cool to $40^{\circ} \mathrm{C}$ prior to the first oxidation step, noted as the " 1 torr cool" condition in Figure 3.6. A comparison of representative spin signals detected in the standard NLSV controls with $1.5 \mu$ m diffusion channels for each of these TB oxidation conditions is presented in Figure 3.6: a-d. 
The identification of TB oxidation conditions enabling acceptable signal to noise ratios in the standard NLSV control devices is performed to establish fabrication procedures for HDDSV devices with increased probability of signal detection. Spin signal characterization is performed through voltage measurements on the nonlocal side of devices ( $\mathrm{V}_{\mathrm{NL}}$ Fig. 3.2: a) as an in plane magnetic field is swept from negative to positive (blue signals in Fig 3.6: a-d) and then positive to negative (orange signals in Fig. 3.6: a-d). This detected voltage is then divided by the injection current, yielding the nonlocal resistance $\left(\mathrm{R}_{\mathrm{NL}}\right)$. The external magnetic field at the maximum negative value aligns both injection and detection FMs to the negative orientation. During the sweep to larger positive values, one FM switches to the positive alignment orientation first and an increased $\mathrm{R}_{\mathrm{NL}}$ value is detected. This increase is a result of the antiparallel alignment of the injection and detection FMs and the spin population imbalance present under the detection FM. As the field strength continues to increase in the positive direction, the second FM becomes positively aligned, and the $\mathrm{R}_{\mathrm{NL}}$ value decreases as the magnets are one again parallel. The width of the magnets, and the associated coercive forces necessary to align them to an external field, is chosen, in this case $200 \mathrm{~nm}$ and $400 \mathrm{~nm}$, to enable a sufficient difference in required field strengths to observe the antiparallel state. ${ }^{136}$ The difference in the $\mathrm{R}_{\mathrm{NL}}$ in the parallel and antiparallel state is the spin signal $\left(\Delta \mathrm{R}_{\mathrm{NL}}\right)$. 


\section{Preliminary oxidation at 75 torr (Fig. 3.6: a) was performed following reported}

results with similar $\mathrm{TiO}_{2}$ TBs and Co FMs however only 1 of 10 characterized devices

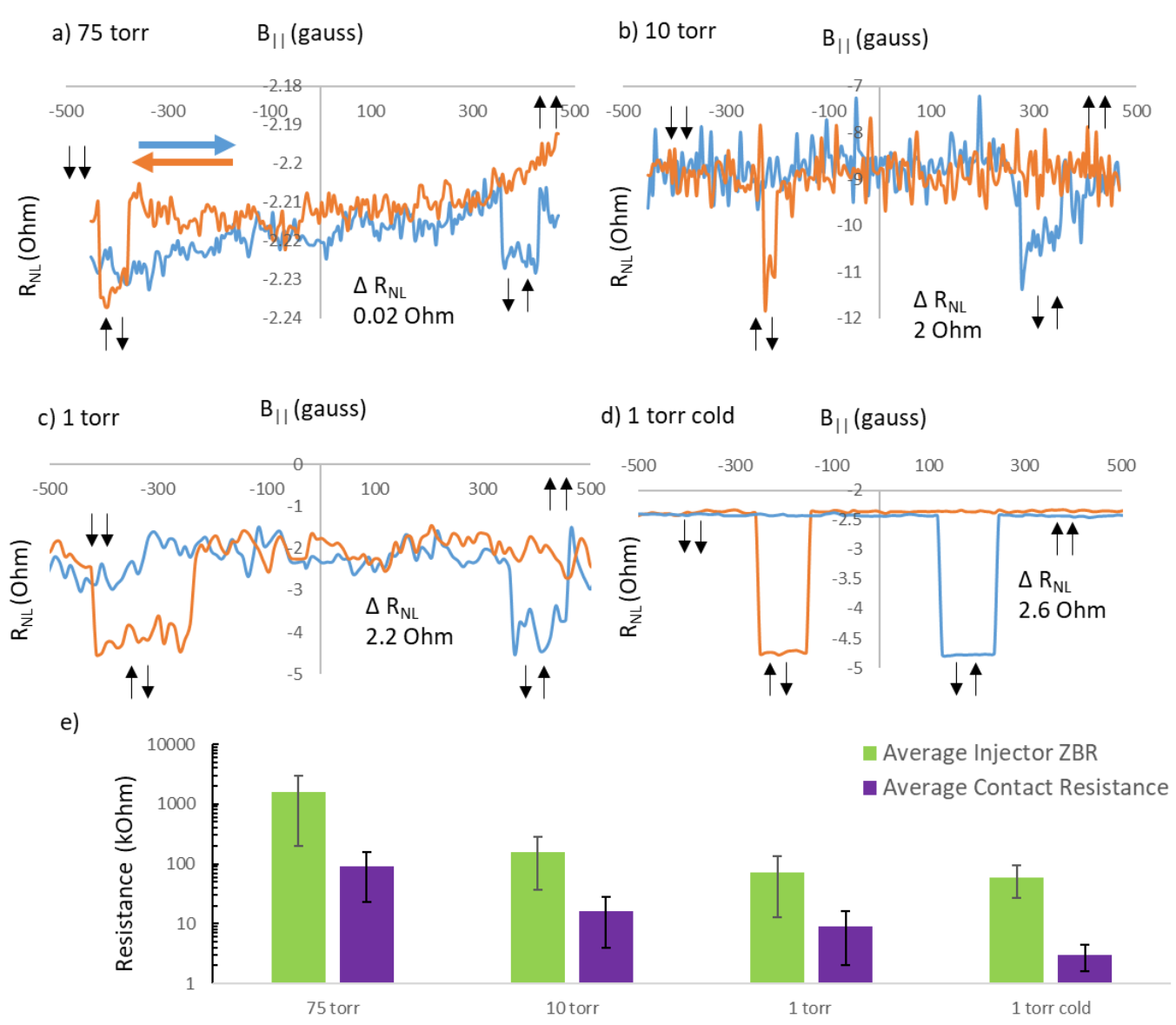

Figure 3.6: a-d) Representative spin signal from NLSV devices with TB oxidation treatments of 75 torr, 10 torr, 1 torr, and 1 torr with cooling prior to $\mathrm{O}_{2}$ exposure. Blue signal represents an external magnetic field sweep from negative to positive while orange signal is collected during a sweep from positive to negative. Black arrows represent the orientation of injection and detection magnets with detected resistance at a maximum in the antiparallel state and a minimum in the parallel state and the spin signal $\left(\Delta \mathrm{R}_{\mathrm{NL}}\right)$ represented by the difference in the antiparallel and parallel configurations. e) Average zero bias resistance of the injection FM/TB contact (green) and metal contact resistance (purple) at each oxidation condition with error bars representing the standard deviation. All data is from NLSV control devices with $1.5 \mu \mathrm{m}$ diffusion channels and 800 $\mathrm{nm}$ graphene channel widths.

exhibited spin signal, $\Delta \mathrm{R}_{\mathrm{NL}}=0.02 \Omega .{ }^{137}$ When the oxidation pressure is reduced to 10 torr

(Fig. 3.6: b), 2 of the 5 characterized devices are observed to exhibit a nonlocal spin 
signal and the average magnitude is increased to $1.8 \Omega$. Following characterization of these devices, current injection was performed via a dedicated current source rather than a voltage source though a $1 \mathrm{M} \Omega$ ballast resistor to increase control of injection current magnitude and reduce noise associated with voltage modulation through highly resistive injector TB/FMs. ${ }^{138}$ Further reduction in oxidation pressure to 1 torr (Fig. 3.6: c) results in a small increase in average detected $\Delta \mathrm{R}_{\mathrm{NL}}$ to $2.1 \Omega$ and all 5 NLSV control devices exhibited spin signal. With the cooling step added to the TB deposition procedure, (Fig. 3.6: d), the background noise is greatly reduced. A comparison of average metal contact resistance to average injector zero bias resistance, (Fig. 3.6: e), indicates that delaying the first oxidation step until the device array has cooled has little effect on the injection FM resistance, from an average of $73 \mathrm{k} \Omega$ to $60 \mathrm{k} \Omega$, but reduces the $\mathrm{MC}$ resistance by a factor of 3 , from $9 \mathrm{k} \Omega$ to $3 \mathrm{k} \Omega$. These findings are in agreement with reports that it is not only the oxidation level of the FM TBs that effect background noise but the relationship between the resistance of the metal contacts and the injection FM/TB. ${ }^{17}$ Specifically, these results indicate that oxidation of the metal contacts or the graphene MC interface, deposited in a previous fabrication step, is dominating device resistance.

The results presented in Figure 3.6 are collected from NLSV control devices with patterned graphene channel widths of $800 \mathrm{~nm}$ as devices with $400 \mathrm{~nm}$ wide patterned channels showed no repeatable spin signal and an increased tendency to present as opens after initial characterization. Scanning electron microscopy (SEM) inspection of graphene channels (Fig. 3.7: a,b) indicates that the graphene channels are on average $250 \mathrm{~nm}$ and $300 \mathrm{~nm}$ thinner than the EBL patterned widths of $800 \mathrm{~nm}$ and $400 \mathrm{~nm}$ respectively (Fig. 


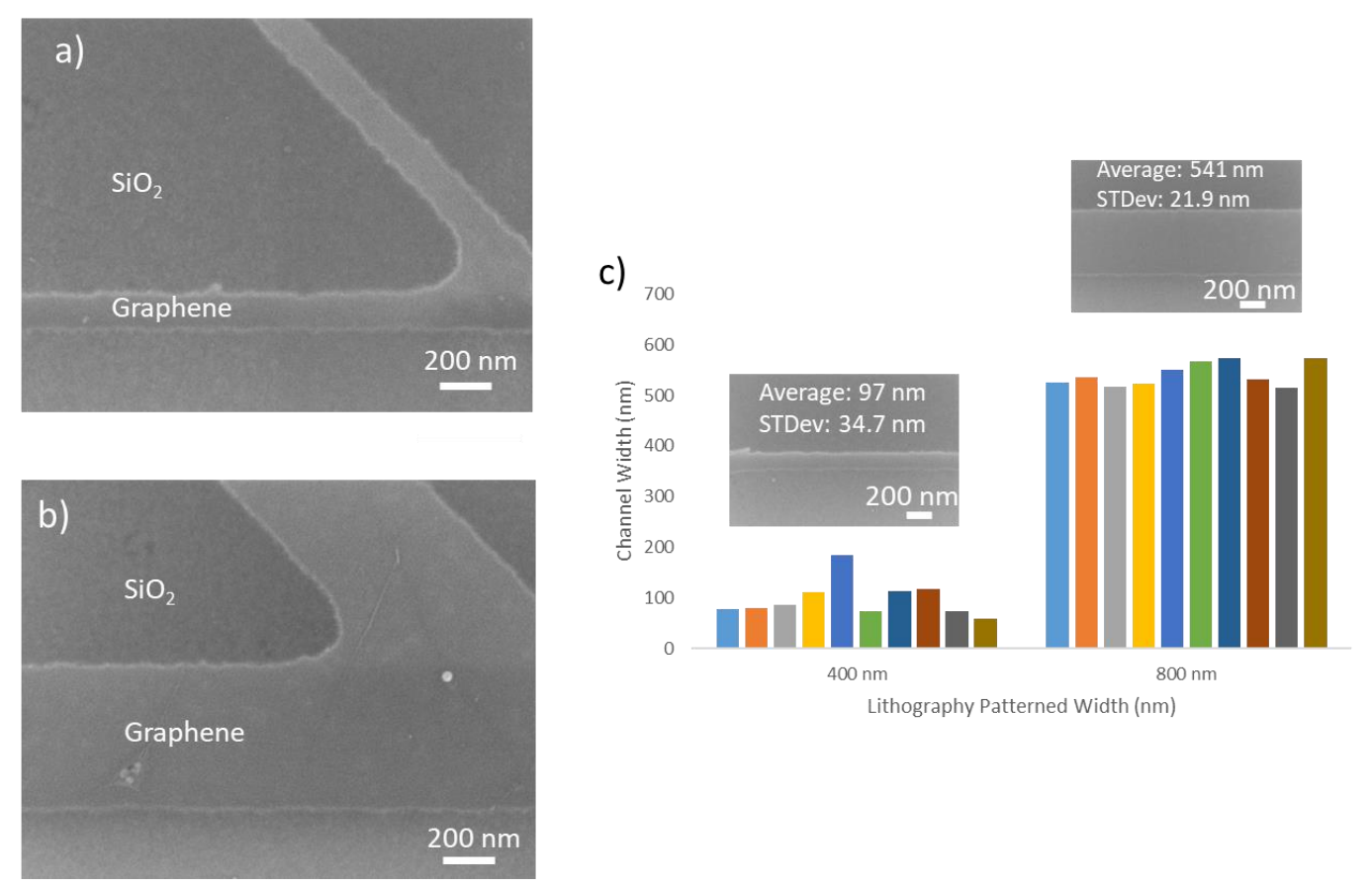

Figure 3.7: SEM images of a) $400 \mathrm{~nm}$ and b) $800 \mathrm{~nm}$ patterned graphene transport channels in HDDSV devices indicating a varied sizes and profiles at each size. c) Comparison of measured widths of $10400 \mathrm{~nm}$ patterned channels and $10800 \mathrm{~nm}$ patterned channels with inset SEM images of channels and average widths.

3.7: c). The discrepancy between patterned width and actual graphene channel width can be attributed to $\mathrm{O}_{2}$ plasma penetration under the lithography mask during the etch step to define the graphene channel. The $400 \mathrm{~nm}$ patterned channels have smaller contact areas between the graphene and the resist mask which may enable further penetration of the $\mathrm{O}_{2}$ molecules and larger variations in channel width, when compared to the $800 \mathrm{~nm}$ patterned channels. The small graphene channel widths on $400 \mathrm{~nm}$ pattered channels warrants further reduction in oxidation pressure during the TB deposition step to realize FM injector contact resistances in the ranges shown to enable spin detection on $550 \mathrm{~nm}$ channels. 
Detected spin signals were observed to increase in magnitude, $\sim 15.2 \%$, over 3 days of characterization accompanied with an increase in both FM injector and metal contact resistances, a phenomenon that has been previously observed in our group. ${ }^{139}$ This increase can be attributed to further oxidation of the Ti portions of both the TB and metal contacts as well as potential oxidation of the Co FM contacts. ${ }^{140}$ To investigate this effect, the devices, which are characterized in a vacuum probe station at $1 \times 10^{-5}$ torr, were exposed to a secondary oxidation procedure. This was achieved through exposure of the device array to ambient conditions for 3 hours and characterization following a return to

\begin{tabular}{|c|c|c|c|}
\hline Parameter & $\begin{array}{l}\text { Pre ambient } \\
\text { exposure } \\
\text { (avg. / SD) }\end{array}$ & $\begin{array}{l}\text { Post ambient } \\
\text { exposure } \\
\text { (avg. / SD) }\end{array}$ & $\begin{array}{l}\% \\
\text { Change }\end{array}$ \\
\hline Inj ZBR (k $\Omega$ ) & $60.3 / 10.8$ & $84.6 / 19.4$ & 40.3 \\
\hline Contact resistance $(\mathrm{k} \Omega)$ & $3.1 / 2.3$ & $6.9 / 4.3$ & 122.5 \\
\hline $\begin{array}{l}\text { Antiparallel state width } \\
\text { (gauss) }\end{array}$ & $185.2 / 12.4$ & $73.9 / 20.6$ & -60.0 \\
\hline Switch location (gauss) & $115.2 / 34.6$ & $389.4 / 183.0$ & 237.5 \\
\hline $\begin{array}{l}\text { Parallel orientation } \\
\text { noise }(\Omega)\end{array}$ & $1.4 / 0.3$ & $3.3 / 2.1$ & 129.1 \\
\hline$\Delta \mathrm{R}_{\mathrm{NL}}(\Omega)$ & $2.4 / 1.0$ & $7.3 / 2.3$ & $\begin{array}{r}93.3 \\
-40\end{array}$ \\
\hline
\end{tabular}

Figure 3.8: Comparison of NLSV control device parameters before and after ambient exposure for 3 hours.

vacuum. A comparison of relevant device parameters collected from NLSV control samples is presented in Figure 3.8 with representative magnetic sweep data pre and post ambient exposure displayed in Figure 3.9. A comparison of the contact resistances pre and post ambient exposure reveals a $40.3 \%$ increase in average injector ZBR and a $122.5 \%$ increase in average metal contact resistance indicating that these exposed contact components are susceptible to further oxidation. Additionally, an increase in the average magnetic field strength at which antiparallel states is observed coupled with a reduction 
in average antiparallel state widths. This may indicate further oxidation of the Co FM contacts with a reduced size of unoxidized Co requiring a stronger external magnetic

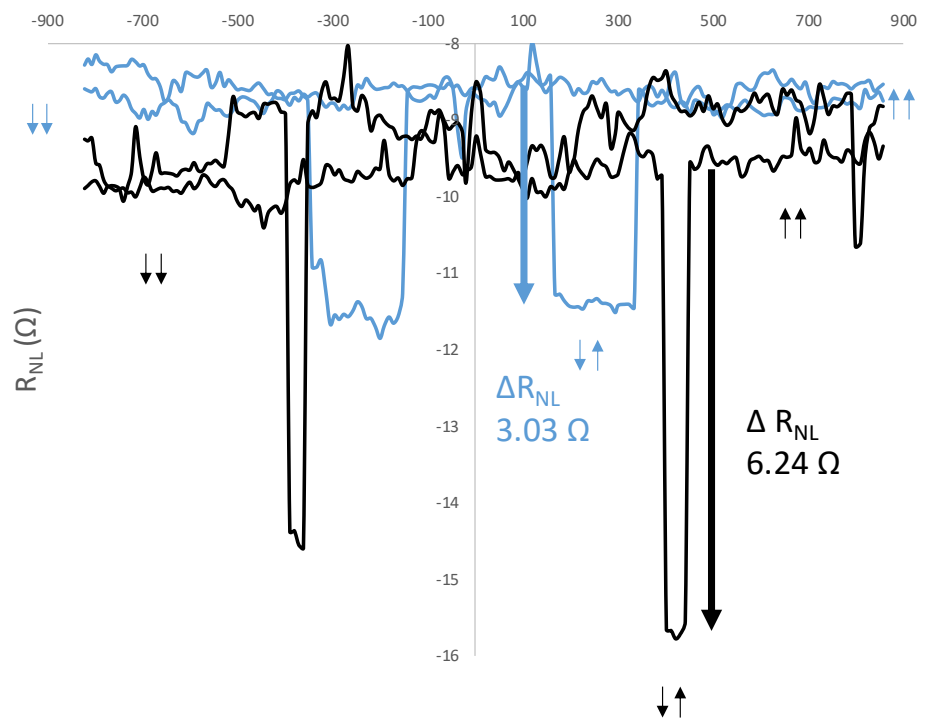

Figure 3.9: Magnetic sweep data displaying pre ambient exposure (blue) and post ambient exposure (black) signals indicating an increase in $\Delta \mathrm{R}_{\mathrm{NL}}$ and a decrease in the width of antiparallel states following exposure to ambient conditions.

field to align. Similarly, the reduction in width of the antiparallel state may stem from in a reduction in the size difference between the FM contacts indicating that they oxidize at varied rates. Finally, a comparison of the post ambient exposure contact resistances and detected spin signals to those characterized in Figure 3.3.3 reveal that changes during ambient exposure of fabricated devices are not achieved through variation of the initial oxidation of the TB during fabrication. These findings indicate that changes to both the Ti portions of contacts and potentially the Co oxidation state and size represent additional avenues for spin signal manipulation and device state targeting beyond the TB/FM fabrication step. ${ }^{141}$ 
The techniques developed in this section demonstrate wafer scale graphene device fabrication and the identification of processing effects on achievable yield. Further optimization of device component parameters and TB/FB targeting coupled with characterization of post fabrication aging effects is ongoing.

\section{4: HDDSV Device Characterization}

In this section, characterization results from post ambient exposure HDDSV devices and associated controls are reported to identify spin transport phenomena. Of the 20 control devices with angled transport channels, none display repeatable spin signal in the nonlocal configuration. There are a number of potential causes for the lack of spin signal detected in the devices. Inspection of the FM injector and detector spacing (Fig. 3.10) reveals a reduced separation in both angled devices compared to the standard NLSV configuration. As local magnetic fields surrounding FM contacts have been proposed as a mechanism for spin relaxation, the additional portion of the graphene channel in proximity to the FM contacts may reduce achievable spin transport distances. ${ }^{142}$ Additionally, as the magnetic field is swept (directions for positive and negative sweeps displayed as black arrows in Fig. 3.10: a) to generate the parallel and antiparallel configuration in the FM contacts, diffusing electron in the channel will travel with a component parallel to the changing field as opposed to perpendicular to it, as in 

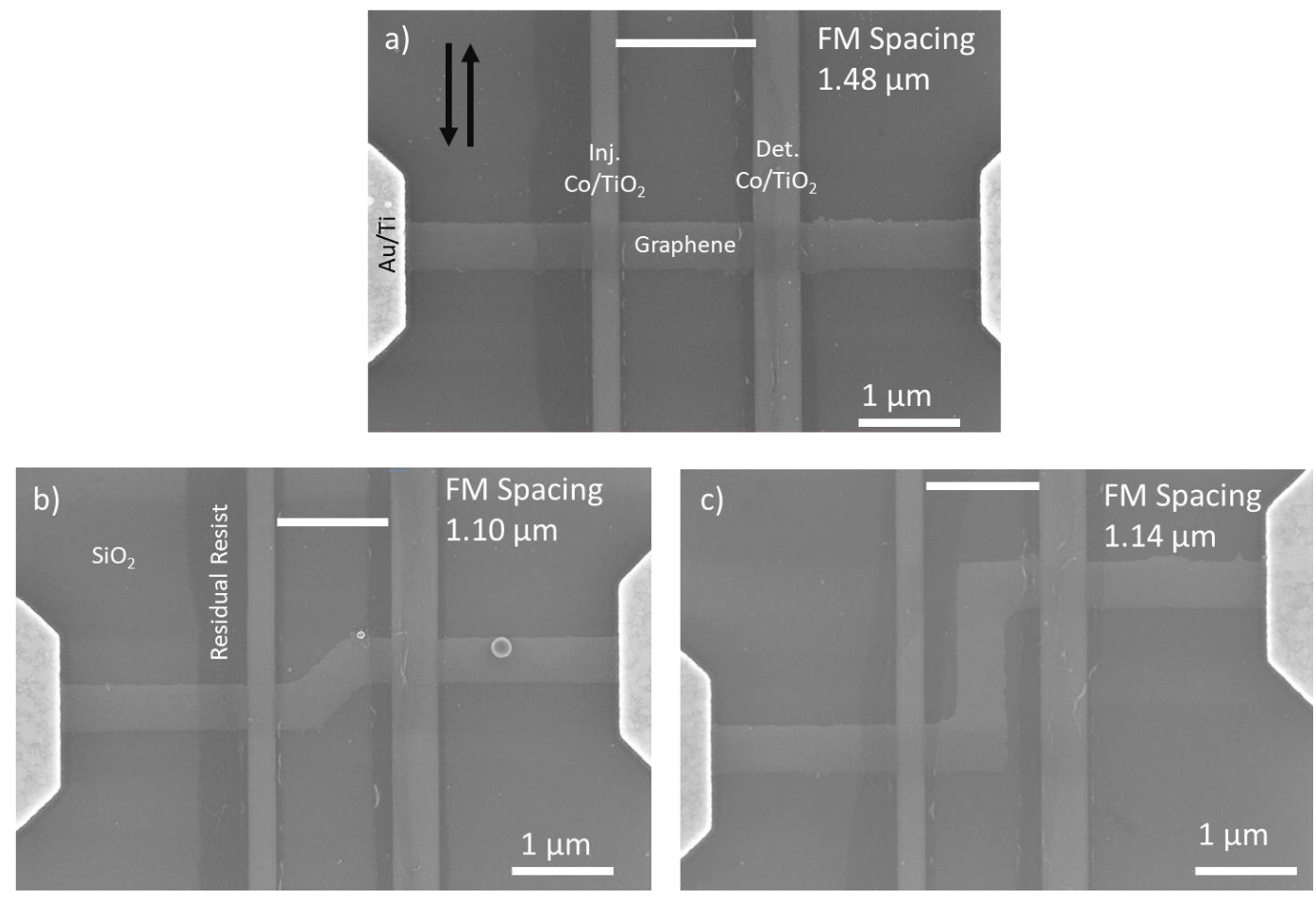

Figure 3.10: SEM images of NLSV control devices a) in the standard configuration with black arrows indicating the direction of the in-plane magnetic sweep, b) with a 45-degree angle in the graphene channel between the injection and detection FM contacts, and c) with a 90-degree angle between injection and detection FM contacts.

the standard NLSV. The effects of an in-plane angle between a diffusing electrons spin polarization and an applied magnetic field are not well described in literature and may represent an additional spin relaxation mechanism similar to the phenomena behind Hanle spin precession measurements. Finally, the increased likelihood of diffusing electron interactions with graphene channel edges in the angled devices may represent increased momentum scattering events, the dominant cause of spin relaxation proposed in the Elliott-Yafet model. Decoupling these effects through modifications in applied external field orientations, increased channel widths and magnet separations, as well as number of bends in the channel will be necessary to determine the dominant phenomena. ${ }^{143}$ 
Additionally, residual resist is detected at the edges of FM contacts for all HDDSV and control devices (Fig. 3.10: b). This residual polymer can be attributed to the small feature size of the FM contacts as well as potential changes to the polymer resist structure during the $\mathrm{O}_{2}$ potion of TB deposition, as it does not appear in the larger metal contacts that do not include an oxidation step during fabrication. The development of post fabrication cleaning procedures to remove this residue is ongoing, however its presence on both HDDSV and control NLSV devices coupled with the reduced conductivity of PMMA compared to graphene indicates a minimal effect on spin transport phenomena.

HDDSV devices were first characterized in the standard nonlocal configuration to determine effects of additional FM contact inclusion (Fig. 3.11). 35\% of HDDSV devices were found to exhibit spin signal in 90-degree angle devices following ambient exposure while $55 \%$ of 45 -degree angle devices demonstrated nonlocal spin signals. Magnetic sweep data from 4 of the 20 characterized HDDSV devices with a 90-degree channel bend displays single switching event (Fig. 3.11: a). As the magnetic field is swept from negative to positive values (blue data in Fig. 3.11: a) one FM contact switches to the positive alignment but the achievable field strength ( \pm 1000 Gauss) is insufficient to align the second magnet and the injection and detection FMs remain in the antiparallel state. 

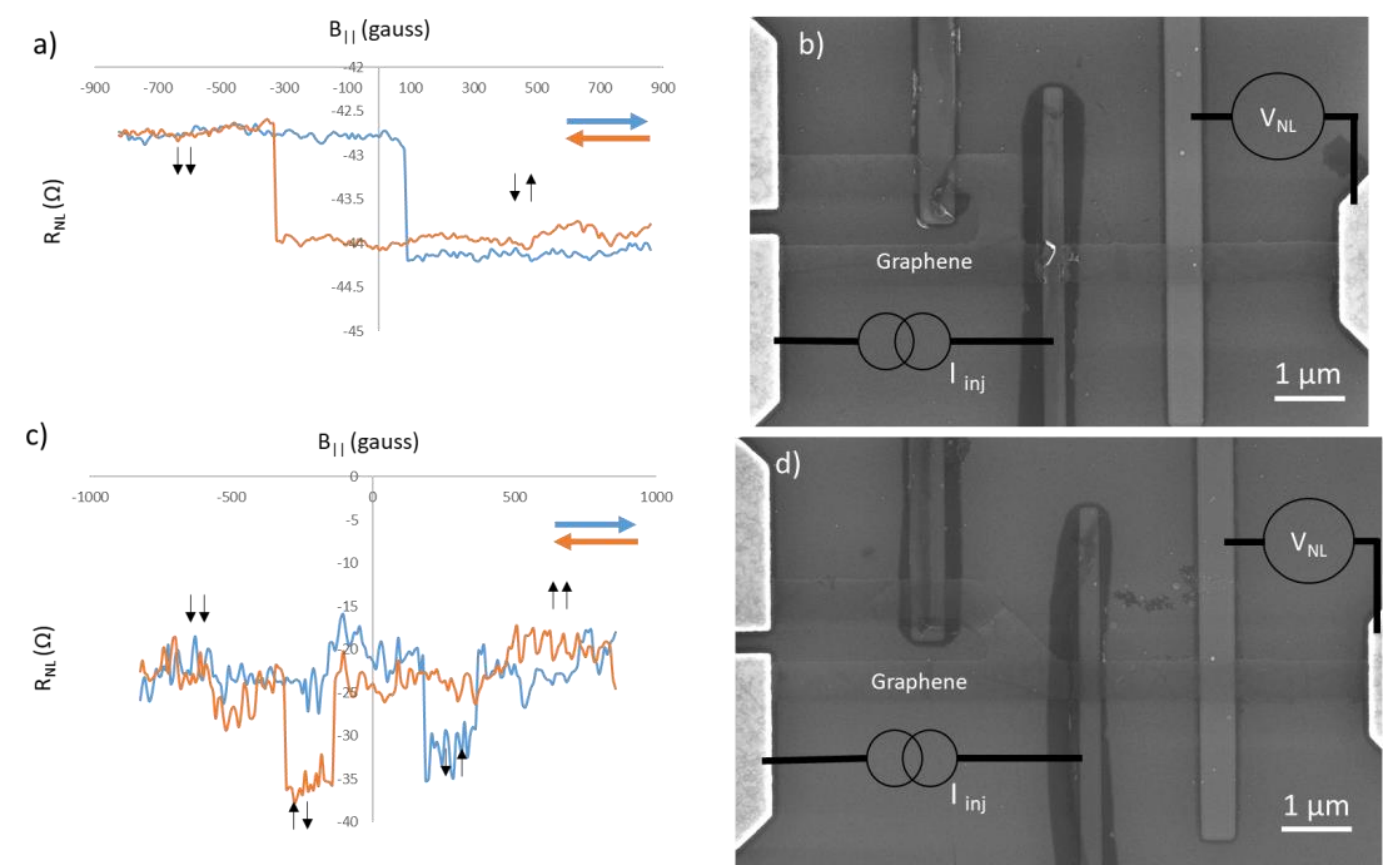

Figure 3.11: a) magnetic sweep profile of 90-degree HDDSV device measured in the standard configuration b) with spin injection and detection occurring on the straight graphene channel. c) magnetic sweep profile of 45-degree HDDSV device measured in the standard configuration d) with spin injection and detection occurring on the straight graphene channel.

During the sweep from positive to negative (orange data in Fig. 3.11: a) the magnet with the positive alignment returns to the negative alignment and the FM injection and detection magnets are again in the parallel orientation. The SEM image (Fig. 3.11: b) of 90-degree devices reveals reduced spacing between the injection FM and the detection FM on the angled channel when compared to the 45 degree devices (Fig. 3.11: d). One potential explanation for the single switch behavior observed in 90-degree devices that is not observed in 45 degree devices (Fig. 3.11: c) is that the proximity of the second detection FM stabilizes the injection FM. As both detection FMs are the same size, they are expected to switch at similar field strengths, and in the antiparallel state with the injection FM, would represent a 3 magnet system. This system would require increased 
field strengths to align the third magnet into the parallel state compared to a 2 magnet system. This is supported by the difference in the external field required to switch into the antiparallel state, 108 gauss, and the parallel state, -305 gauss. Additionally, while the HDDSV devices with 45 degree angles show traditional switching behavior (Fig. 3.11: c), from parallel to antiparallel states for each external field sweep direction, the average antiparallel width is wider, 135 gauss, and is centered at a lower external field, 167 gauss in the positive direction, when compared to standard NLSV devices, 73 and 383 gauss respectively. The single switch and increased stability of the antiparallel orientation observed in the 90 degree channels reveals a potential avenue for increased targeting of switched states through the use of stabilization magnets near injection and detection FMs. ${ }^{144}$

The angled side of HDDSV devices were characterized to detect spin signals in the asymmetric channel which are proposed to originate from a moving population of spin polarized electrons. In the HDDSV configuration, no 90-degree devices exhibited nonlocal spin signal while $20 \%$ of 45 -degree devices exhibited repeatable spin transport. Nonlocal voltage measurements were performed, $\left(\mathrm{V}_{\mathrm{NL}}\right.$ in Fig. 3.12: a) and large spin signals, average $\Delta R_{N L}$ of $742 \Omega$ across the 3 active devices, were detected (Fig. 3.11: b). In addition to the detection of large spin signals, increased background resistance in the parallel alignment was observed, averaging $215 \Omega$, while antiparallel orientations occurred at increased external field strengths, averaging 815 gauss. The increased 
background resistance could stem from current leakage down the asymmetric leg, representing a local measurement, and large spin signals have been reported in literature,

a)

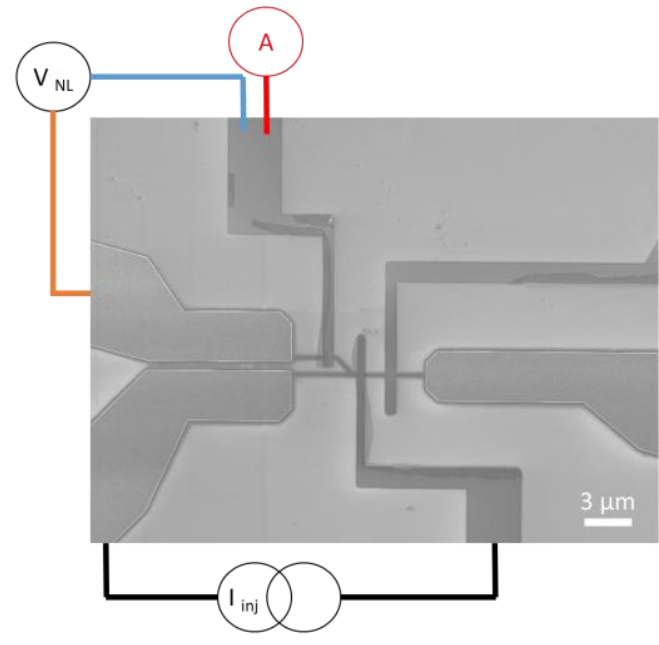

b)
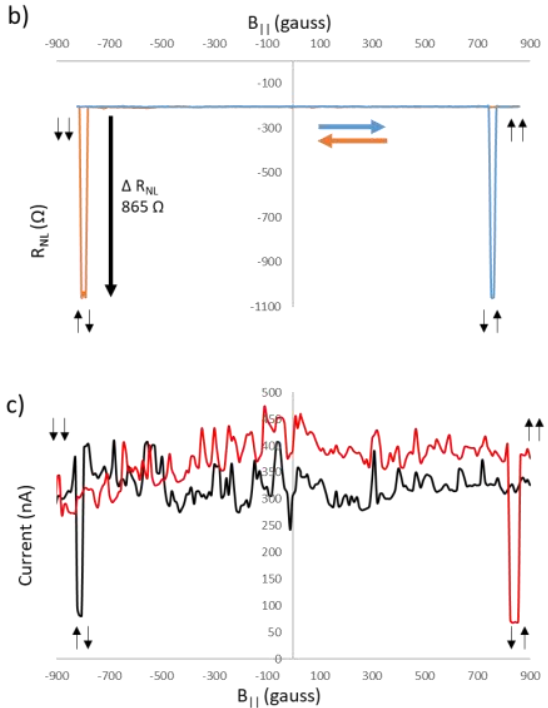

Figure 3.12: a) SEM image of HDDSV device with 45-degree asymmetric angle and overlaid depiction of nonlocal $\left(\mathrm{V}_{\mathrm{NL}}\right)$ and local (A) measurement configurations. b) Nonlocal magnetic sweep measurements at the HDDSV channel with $\Delta \mathrm{R}_{\mathrm{NL}}$ of $865 \Omega$. c) Local measurement magnetic sweep measurements depicting current reduction in the antiparallel state.

on the order of $\mathrm{M} \Omega \mathrm{s}$, in the local configuration with $\mathrm{M} \Omega$ resistance injection and detection contacts. ${ }^{145}$ To determine the potential for this device to act in a local configuration, current measurements were taken at the HDDSV detection FM ( red A in Fig. 3.11: a) while $1 \mu \mathrm{A}$ current was sourced from the injection FM to the grounded MC. Current measurements as the applied external field is swept (Fig. 3.11: c) reveal switching between the parallel and antiparallel states in this local testing configuration and the $369 \mathrm{nA}$ average current in the parallel FM state confirms the current divider operation with a more resistive detection FM than grounded MC. As the external magnetic field is swept, and the injection and detection FMs become antiparallel, the resistance at the detection FM to the spin polarized electrons sourced from the injection 
FM increases and the detected current decreases. The spin signal is not observed in a 2 terminal local measurement, with current monitoring at the HDDSV detection FM as voltage is applied between the injection and detection contacts. The underlying mechanism enabling spin signal detection in 3 terminal local measurements but not in a 2 terminal configuration requires further investigation. While current switching with varying applied voltages in carbon nanotube $y$-shaped devices has been observed ${ }^{146}$ and $\mathrm{y}$-shaped channel preference of spin polarized electrons in 2D materials with and without Rashba spin orbit coupling has been theoretically predicted ${ }^{147}$, the detected phenomena in HDDSV devices presented here has not been observed previously. The large detected spin signal in the HDDSV channel and 3 terminal local measurement capabilities represent a potential avenue for long distance spin transport and reduced device complexity, with a single injection channel and branching detection channel addressable in a three probe configuration as opposed to the 4 required in standard NLSV devices. Though these preliminary results are promising, deconvolution of drift and diffusion effects, FM and metal contact resistance ratios, spin scattering at the asymmetric intersection, and in-plane angle effects between the applied external magnetic field and the spin polarized electron transport direction will be necessary to advance graphene as a spin transport channel for spintronic applications. The HDDSV device reported in this section represents a new configuration for characterization of these effects.

\section{5: Summary}

In conclusion, we have demonstrated the design and fabrication of wafer scale graphene spin transport devices through scalable processes with $64 \%$ graphene channel 
yields over 360 devices. 120 device arrays containing standard NLSV devices, angled channel controls, and HDDSV devices with varied parameters were designed to reveal graphene spin transport phenomena not readily observable in current device architectures. Standard NLSV devices were used to optimize tunnel barrier oxidation procedures and enabled $95 \%$ yield of spin active control devices with $\sim 550 \mathrm{~nm}$ graphene channels. Further oxidation of both FM and metal contacts were performed and revealed an average $193 \%$ increase to achievable spin signal magnitude post ambient exposure and a reduction in the average antiparallel state width with an increase in the average external field strength required to flip FM magnetic orientations. The results from post fabrication ambient exposure reveal detected spin signal magnitudes and profiles not achievable through oxidation variation at the time of tunnel barrier fabrication, pointing to additional fabrication techniques for spin signal manipulation. Control devices with angled diffusion channels show no repeatable spin signal and suggest FM influences, graphene edge scattering, and in-plane angles between diffusing electrons and the external magnetic field as potential causes for early spin relaxation. While neither 45 -degree or 90-degree angled control devices displayed a spin signal, some HDDSV devices with 45-degree angles displayed the large spin signals, with a maximum $\Delta \mathrm{R}_{\mathrm{NL}}$ of $865 \Omega$. These devices also displayed magnetic switching behavior in a local three-point measurement configuration indicating that some interplay between injected and diffusing electrons may be enabling spin signal detection not observed in the angled control devices. Finally, the standard nonlocal side of HDDSV devices display larger antiparallel state widths on average compared to their control NLSV counterparts while $20 \%$ of 90 -degree HDDSV 
devices display single switch events. This stabilization of magnetic orientations may result from the additional FM included in the HDDSV devices and represents a potential avenue for FM state targeting without altering contact sizes through the inclusion of additional FM or permanent magnet device components. This work represents the large scale fabrication of new graphene spin device architecture and identifies avenues for further fabrication improvements and device considerations necessary for the realization of spintronic devices with graphene transport channels.

\section{6: Experimental Methods \\ Wafer scale Graphene Device Fabrication}

\section{5-inch by 2.5-inch graphene films on 4 inch $\mathrm{SiO}_{2}$ were purchased through}

Graphene Square. Electron beam lithography (EBL) was performed by a collaborator at Intel Corp. to define graphene channels and contact locations. Graphene removal to define channels was performed in a custom CVD reactor through exposure to a $20 \mathrm{~W} \mathrm{O}_{2}$ plasma for 15 seconds and graphene quality was verified through Raman inspection in a Horiba Jobin Yvon HR800 with 532 nm laser excitation. Metal depositions were performed at Portland State University on a Kurt. J Lesker AXXISS multi-target physical vapor deposition system (PVD) via electron beam evaporation. Metal contacts were fabricated through $5 \mathrm{~nm}$ Ti deposition followed by deposition of $50 \mathrm{~nm}$ Au at $1 \times 10^{-7}$ torr. Forming gas anneal procedures were performed on a custom CVD chamber at $300^{\circ} \mathrm{C}$ for 2 hours under an atmosphere of $95 \% \mathrm{~N}_{2} / 5 \% \mathrm{H}_{2}$ at a pressure of $5 \times 10^{-4}$ torr after liftoff of metal contacts. Atomic force microcopy inspection of graphene surface was performed with a Nanosurf Flex-Axiom system in noncontact mode. Following EBL definition of 
FM contact locations at Intel Corp., the sample was heated in the PVD system to $120^{\circ} \mathrm{C}$ for 2 minutes and TBs were deposited via EBE of $0.4 \AA$ of Ti followed by in situ oxidation at 75 torr, 10 torr, 1 torr, or 1 torr after the sample had cooled to $40^{\circ} \mathrm{C}$, for 10 minutes. The PVD was evacuated to base pressure following oxidation and the $0.4 \AA \mathrm{Ti}$ deposition and oxidation steps were repeated. FM contacts were deposited via EBE of 25 nm of Co and lift off was performed in acetone for 1 hour. Following lift off the sample was placed in to the probestation at $1 \times 10^{-5}$ torr for characterization.

\section{Device Characterization}

Characterization of graphene channels following metal contact deposition was performed on a Cascade Microtech probe station with 2-point and 4-point DC measurements taken with an Agilent B1500A semiconductor parameter analyzer (SPA) to determine graphene and metal contact electrical properties. Following FM deposition, the sample was characterized in a Lakeshore vacuum probe station with in-plane magnetic field capabilities of \pm 1000 gauss. Spin signals were detected through sweeping the magnetic field and detecting current or nonlocal voltages with an SRS SR850 DSP lock-in amplifier. Current was first injected through voltage modulation driven by the lock-in amplifier sine output through a $1 \mathrm{M} \Omega$ ballast resistor, however increased current control was achieved with current driven by a Keithly 6221a current source linked to the lock-in amplifier and was used to characterize HDDSV devices. A back gate voltage was applied to the sample at $40 \mathrm{~V}$ through the SPA during characterization of magnetic sweeps and data was collected through an in-house LabVIEW program to record voltage or current as a function of in-plane magnetic field strength. 


\section{4: Conclusion}

Graphene adoption in CMOS and beyond-CMOS applications necessitates advancements in synthesis and characterization techniques to reduce growth requirements and reveal phenomena governing spin transport. High temperature CVD processes on micrometer scale $\mathrm{Cu}$ remains the primary method for large scale and high quality graphene synthesis. The temperature and catalyst requirements associated with this technique have hindered the inclusion of graphene as a component in CMOS applications seeking to utilize the single atomic thickness material as an alternative to current device components. In the case of beyond-CMOS applications, the uncertainty associated with fundamental spin transport mechanisms as well as the lack of varied device configurations produced through scalable fabrication techniques and materials has slowed the transition from fundamental investigation to implementation. The findings reported here demonstrate the development of PECVD graphene synthesis techniques to reduce growth requirements toward advancing graphene adoption in current CMOS applications, while novel device configurations fabricated through scalable processes reveal spin transport phenomena representing avenues toward the realization of graphene spintronic devices.

Following the initial demonstration of graphene synthesis via CVD, intensive research efforts have focused on reducing catalyst thickness and synthesis temperature requirements toward direct incorporation of graphene in applications with nonplanar surfaces where transfer techniques are precluded. Toward this goal, the synergistic relationship between the reaction environment, hydrocarbon precursor configuration, and 
catalyst morphology and reactivity, has been extensively studied. However, maintaining graphene film uniformity and quality remain as challenges as synthesis temperatures and catalyst thicknesses are reduced. To identify new avenues for continued advancement, this dissertation described two pathways, 1) control of hydrocarbon precursor size and ionization state during PECVD synthesis and 2) multimetal catalyst design, to enable targeted growth pathways and resultant graphene morphology.

We have demonstrated $95 \%$ few-to-monolayer graphene at $500^{\circ} \mathrm{C}$ through increasing the hydrocarbon precursor size and ionization state during PECVD synthesis. The characterization of hydrocarbon species generated during plasma ignition and control of species interacting with the catalyst, represents a new methodology for targeted graphene synthesis. The ability to selectively screen high energy ions and allow larger neutral molecules to interact with the catalyst enables reduced rates of multilayer graphene formation. Additionally, increasing the energy of these larger molecules through secondary plasma generation enables increased film uniformity and reduced defect concentrations. While these techniques have been used to produce quality graphene on $50 \mathrm{~nm}$ metal catalysts, they demonstrate a new technique for control of graphene synthesis pathways that may enable direct graphene formation on new substrates through matching precursor reactivity and size to the target substrate characteristics. Finally, the ability to control defect densities through manipulation of the hydrocarbon precursor during synthesis represents a new method for synthesizing defective graphene, which exhibits unique properties dependent on defect types and concentrations, for targeted applications. 
In addition to in situ PECVD synthesis manipulation, we have demonstrated the use of trimetallic catalysts to enhance graphene layer number and uniformity control over traditionally considered bimetallic catalysts. Simulation reveals that Au inclusion in primarily $\mathrm{Ni}$ catalysts increases energy requirements for hydrocarbon dehydrogenation while $\mathrm{Cu}$ inclusion increases energy requirements for carbon-carbon bond decomposition. The combination of small percentages of these metals, in a bulk Ni catalyst, increases energy requirements for all hydrocarbon decomposition steps, suggesting a mechanism for observed improvements in graphene layer number and uniformity grown on $\mathrm{NiAuCu}$ catalysts. Grain size and orientation inspection of these catalysts reveals modulation with each metal component inclusion and represents an avenue toward targeting graphene morphology via catalyst composition design. Through modulating the rate of hydrocarbon precursor decomposition, the rate of both graphene synthesis and catalyst saturation can be tuned to promote layer number suppression and increases to film uniformity, the primary issues associated with low temperature graphene production on thin film catalysts. These synthesis and catalyst design techniques represent new avenues toward promoting graphene inclusion in industry applications.

In addition to the development of graphene synthesis procedures, graphene spin transport phenomena have been considered. Graphene as a spin transport channel is primarily investigated in a NLSV device configuration which enables determination of spin transport parameters through detection of a non-local voltage arising from spin diffusion. Experimental results collected from these devices are orders of magnitude smaller than those theoretically predicted and numerous causes of this discrepancy have 
been proposed in literature. While increases in achievable spin transport in a graphene channel have been realized through inclusion of additional materials and external field application, the standard device design and small numbers of devices considered warrants the use of novel device configurations and the demonstration of scalable fabrication techniques and materials.

To advance understandings of graphene spin transport phenomena and device configurational effects, a new device architecture, the HDDSV, has been developed. This device contains an additional graphene channel originating from the injection leg of a standard NLSV device and is intended to reveal diffusion and drift interactions as well as magnetic component effects on achievable spin signals. 36 arrays, each containing 120 devices has been fabricated through industry standard lithography and metal deposition techniques on a 4-inch wafer of commercially available graphene to demonstrate scalable processes suitable for spin based device production.

Characterization of non-local spin signals in the standard leg of some HDDSV configurations reveals an increase in state stability of the antiparallel magnet orientation. This phenomenon may be attributed to the inclusion of additional magnetic contacts in the HDDSV device, which represents an avenue for improved precision in targeting states of individual detection components in devices with increased complexity. Examination of detected spin signals in the additional leg of some HDDSV devices with 45-degree angles reveal large spin signals, $\Delta \mathrm{R}_{\mathrm{NL}}$ up to $865 \Omega$, with spin signal detected in a 3-point local configuration. The detection of these spin signals, compared to the lack of spin signals detected in angled control devices or 90-degree HDDSV devices, indicates a 
potential interaction between drift and diffusing electrons. Additionally, the 3-point local measurement capabilities of this configuration represents an avenue for the development of long distance spin transport devices through a single injection channel and multiple detection channels. While these results are promising, the new device configuration reveals a variety of phenomena not observed in the standard NLSV configuration, ranging from graphene edge interactions in angled channels, increased local magnetic field complexity with additional magnetic components, as well as a variation in electron diffusion direction, spin polarization direction, and external magnetic field direction that warrant further investigation.

The findings reported in this dissertation represent not only the detection of new phenomena but the development of techniques to enable continued advancement of graphene synthesis and characterization toward application. The in situ manipulation of PECVD precursors to control synthesis pathways to the characteristics of the target substrate represents an avenue for graphene synthesis on an expanded range of materials. Additionally, the detection of spin signals in HDDSV devices in a local 3-point configuration reveals a new device architecture for optimization toward the realization of graphene spintronics. Continued research in these areas is expected to advance graphene inclusion in both current and future applications. 


\section{References}

(1) Novoselov, K. S.; Geim, A. K.; Morozov, S. V.; Jiang, D.; Zhang, Y.; Dubonos, S. V.; Grigorieva, I. V.; Firsov, A. A. Electric Field Effect in Atomically Thin Carbon Films. Science 2004, 306 (5696), 666-669. https://doi.org/10.1126/science.1102896.

(2) Wallace, P. R. The Band Theory of Graphite. Phys. Rev. 1947, 71 (9), 622-634. https://doi.org/10.1103/PhysRev.71.622.

(3) Novoselov, K. S.; Geim, A. K.; Morozov, S. V.; Jiang, D.; Katsnelson, M. I.; Grigorieva, I. V.; Dubonos, S. V.; Firsov, A. A. Two-Dimensional Gas of Massless Dirac Fermions in Graphene. Nature 2005, 438 (7065), 197-200. https://doi.org/10.1038/nature04233.

(4) Lee, C.; Wei, X.; Kysar, J. W.; Hone, J. Measurement of the Elastic Properties and Intrinsic Strength of Monolayer Graphene. Science 2008, 321 (5887), 385-388. https://doi.org/10.1126/science.1157996.

(5) Mayorov, A. S.; Gorbachev, R. V.; Morozov, S. V.; Britnell, L.; Jalil, R.; Ponomarenko, L. A.; Blake, P.; Novoselov, K. S.; Watanabe, K.; Taniguchi, T.; Geim, A. K. Micrometer-Scale Ballistic Transport in Encapsulated Graphene at Room Temperature. Nano Lett. 2011, 11 (6), 2396-2399. https://doi.org/10.1021/nl200758b.

(6) Balandin, A. A. Thermal Properties of Graphene and Nanostructured Carbon Materials. Nat. Mater. 2011, 10 (8), 569-581. https://doi.org/10.1038/nmat3064.

(7) Moser, J.; Barreiro, A.; Bachtold, A. Current-Induced Cleaning of Graphene. Appl. Phys. Lett. 2007, 91 (16), 163513. https://doi.org/10.1063/1.2789673.

(8) Huertas-Hernando, D.; Guinea, F.; Brataas, A. Spin-Orbit Coupling in Curved Graphene, Fullerenes, Nanotubes, and Nanotube Caps. Phys. Rev. B 2006, 74 (15). https://doi.org/10.1103/PhysRevB.74.155426.

(9) Qiao, Z.; Ren, W.; Chen, H.; Bellaiche, L.; Zhang, Z.; MacDonald, A. H.; Niu, Q. Quantum Anomalous Hall Effect in Graphene Proximity Coupled to an Antiferromagnetic Insulator. Phys. Rev. Lett. 2014, 112 (11), 116404. https://doi.org/10.1103/PhysRevLett.112.116404.

(10) Fu, L.; Kane, C. L. Superconducting Proximity Effect and Majorana Fermions at the Surface of a Topological Insulator. Phys. Rev. Lett. 2008, 100 (9). https://doi.org/10.1103/PhysRevLett.100.096407.

(11) Shailos, A.; Nativel, W.; Kasumov, A.; Collet, C.; Ferrier, M.; Guéron, S.; Deblock, R.; Bouchiat, H. Proximity Effect and Multiple Andreev Reflections in Few-Layer Graphene. Europhys. Lett. EPL 2007, 79 (5), 57008. https://doi.org/10.1209/02955075/79/57008.

(12) Eda, G.; Fanchini, G.; Chhowalla, M. Large-Area Ultrathin Films of Reduced Graphene Oxide as a Transparent and Flexible Electronic Material. Nat. Nanotechnol. 2008, 3 (5), 270-274. https://doi.org/10.1038/nnano.2008.83.

(13) Das, A.; Pisana, S.; Chakraborty, B.; Piscanec, S.; Saha, S. K.; Waghmare, U. V.; Novoselov, K. S.; Krishnamurthy, H. R.; Geim, A. K.; Ferrari, A. C.; Sood, A. K. Monitoring Dopants by Raman Scattering in an Electrochemically Top-Gated 
Graphene Transistor. Nat. Nanotechnol. 2008, 3 (4), 210-215.

https://doi.org/10.1038/nnano.2008.67.

(14) Roy, S. S.; Arnold, M. S. Improving Graphene Diffusion Barriers via Stacking Multiple Layers and Grain Size Engineering. Adv. Funct. Mater. 2013, 23 (29), 3638-3644. https://doi.org/10.1002/adfm.201203179.

(15) Brownson, D. A. C.; Kampouris, D. K.; Banks, C. E. An Overview of Graphene in Energy Production and Storage Applications. J. Power Sources 2011, 196 (11), 4873-4885. https://doi.org/10.1016/j.jpowsour.2011.02.022.

(16) Stankovich, S.; Dikin, D. A.; Dommett, G. H. B.; Kohlhaas, K. M.; Zimney, E. J.; Stach, E. A.; Piner, R. D.; Nguyen, S. T.; Ruoff, R. S. Graphene-Based Composite Materials. Nature 2006, 442 (7100), 282-286. https://doi.org/10.1038/nature04969.

(17) Han, W.; Kawakami, R. K.; Gmitra, M.; Fabian, J. Graphene Spintronics. Nat. Nanotechnol. 2014, 9 (10), 794-807. https://doi.org/10.1038/nnano.2014.214.

(18) Mortazavi, B.; Ahzi, S. Thermal Conductivity and Tensile Response of Defective Graphene: A Molecular Dynamics Study. Carbon 2013, 63, 460-470. https://doi.org/10.1016/j.carbon.2013.07.017.

(19) Hao, F.; Fang, D.; Xu, Z. Mechanical and Thermal Transport Properties of Graphene with Defects. Appl. Phys. Lett. 2011, 99 (4), 041901. https://doi.org/10.1063/1.3615290.

(20) Gómez-Navarro, C.; Weitz, R. T.; Bittner, A. M.; Scolari, M.; Mews, A.; Burghard, M.; Kern, K. Electronic Transport Properties of Individual Chemically Reduced Graphene Oxide Sheets. Nano Lett. 2007, 7 (11), 3499-3503. https://doi.org/10.1021/n1072090c.

(21) Hernandez, Y.; Nicolosi, V.; Lotya, M.; Blighe, F. M.; Sun, Z.; De, S.; McGovern, I. T.; Holland, B.; Byrne, M.; Gun'Ko, Y. K.; Boland, J. J.; Niraj, P.; Duesberg, G.; Krishnamurthy, S.; Goodhue, R.; Hutchison, J.; Scardaci, V.; Ferrari, A. C.; Coleman, J. N. High-Yield Production of Graphene by Liquid-Phase Exfoliation of Graphite. Nat. Nanotechnol. 2008, 3 (9), 563-568. https://doi.org/10.1038/nnano.2008.215.

(22) Xu, Y.; Sheng, K.; Li, C.; Shi, G. Self-Assembled Graphene Hydrogel via a OneStep Hydrothermal Process. ACS Nano 2010, 4 (7), 4324-4330. https://doi.org/10.1021/nn101187z.

(23) Emtsev, K. V.; Bostwick, A.; Horn, K.; Jobst, J.; Kellogg, G. L.; Ley, L.; McChesney, J. L.; Ohta, T.; Reshanov, S. A.; Röhrl, J.; Rotenberg, E.; Schmid, A. K.; Waldmann, D.; Weber, H. B.; Seyller, T. Towards Wafer-Size Graphene Layers by Atmospheric Pressure Graphitization of Silicon Carbide. Nat. Mater. 2009, 8 (3), 203-207. https://doi.org/10.1038/nmat2382.

(24) Jin, Y.; Hu, B.; Wei, Z.; Luo, Z.; Wei, D.; Xi, Y.; Zhang, Y.; Liu, Y. Roles of H2 in Annealing and Growth Times of Graphene CVD Synthesis over Copper Foil. $J$. Mater. Chem. A 2014, 2 (38), 16208-16216. https://doi.org/10.1039/C4TA02557A.

(25) Novoselov, K. S.; Fal'ko, V. I.; Colombo, L.; Gellert, P. R.; Schwab, M. G.; Kim, K. A Roadmap for Graphene. Nature 2012, 490 (7419), 192-200. https://doi.org/10.1038/nature11458. 
(26) Mattevi, C.; Kim, H.; Chhowalla, M. A Review of Chemical Vapour Deposition of Graphene on Copper. J. Mater. Chem. 2011, 21 (10), 3324-3334.

https://doi.org/10.1039/C0JM02126A.

(27) Yu, Q.; Lian, J.; Siriponglert, S.; Li, H.; Chen, Y. P.; Pei, S.-S. Graphene Segregated on Ni Surfaces and Transferred to Insulators. Appl. Phys. Lett. 2008, 93 (11), 113103. https://doi.org/10.1063/1.2982585.

(28) Chu, J. H.; Kwak, J.; Kwon, T.-Y.; Park, S.-D.; Go, H.; Kim, S. Y.; Park, K.; Kang, S.; Kwon, S.-Y. Facile Synthesis of Few-Layer Graphene with a Controllable Thickness Using Rapid Thermal Annealing. ACS Appl. Mater. Interfaces 2012, 4 (3), 1777-1782. https://doi.org/10.1021/am3000177.

(29) Li, X.; Cai, W.; An, J.; Kim, S.; Nah, J.; Yang, D.; Piner, R.; Velamakanni, A.; Jung, I.; Tutuc, E.; Banerjee, S. K.; Colombo, L.; Ruoff, R. S. Large-Area Synthesis of High-Quality and Uniform Graphene Films on Copper Foils. Science 2009, 324 (5932), 1312-1314. https://doi.org/10.1126/science.1171245.

(30) Han, G. H.; Güneş, F.; Bae, J. J.; Kim, E. S.; Chae, S. J.; Shin, H.-J.; Choi, J.-Y.; Pribat, D.; Lee, Y. H. Influence of Copper Morphology in Forming Nucleation Seeds for Graphene Growth. Nano Lett. 2011, 11 (10), 4144-4148. https://doi.org/10.1021/nl201980p.

(31) Vlassiouk, I.; Regmi, M.; Fulvio, P.; Dai, S.; Datskos, P.; Eres, G.; Smirnov, S. Role of Hydrogen in Chemical Vapor Deposition Growth of Large Single-Crystal Graphene. ACS Nano 2011, 5 (7), 6069-6076. https://doi.org/10.1021/nn201978y.

(32) Ito, Y.; Christodoulou, C.; Nardi, M. V.; Koch, N.; Sachdev, H.; Müllen, K. Chemical Vapor Deposition of N-Doped Graphene and Carbon Films: The Role of Precursors and Gas Phase. ACS Nano 2014, 8 (4), 3337-3346. https://doi.org/10.1021/nn405661b.

(33) Zhang, B.; Lee, W. H.; Piner, R.; Kholmanov, I.; Wu, Y.; Li, H.; Ji, H.; Ruoff, R. S. Low-Temperature Chemical Vapor Deposition Growth of Graphene from Toluene on Electropolished Copper Foils. ACS Nano 2012, 6 (3), 2471-2476. https://doi.org/10.1021/nn204827h.

(34) Chen, L.; Hernandez, Y.; Feng, X.; Müllen, K. From Nanographene and Graphene Nanoribbons to Graphene Sheets: Chemical Synthesis. Angew. Chem. Int. Ed. 2012, 51 (31), 7640-7654. https://doi.org/10.1002/anie.201201084.

(35) Bae, S.; Kim, H.; Lee, Y.; Xu, X.; Park, J.-S.; Zheng, Y.; Balakrishnan, J.; Lei, T.; Ri Kim, H.; Song, Y. I.; Kim, Y.-J.; Kim, K. S.; Özyilmaz, B.; Ahn, J.-H.; Hong, B. H.; Iijima, S. Roll-to-Roll Production of 30-Inch Graphene Films for Transparent Electrodes. Nat. Nanotechnol. 2010, 5 (8), 574-578. https://doi.org/10.1038/nnano.2010.132.

(36) Sharma, K. P.; Shinde, S. M.; Rosmi, M. S.; Sharma, S.; Kalita, G.; Tanemura, M. Effect of Copper Foil Annealing Process on Large Graphene Domain Growth by Solid Source-Based Chemical Vapor Deposition. J. Mater. Sci. 2016, 51 (15), 7220-7228. https://doi.org/10.1007/s10853-016-0003-8.

(37) Weatherup, R. S.; Bayer, B. C.; Blume, R.; Ducati, C.; Baehtz, C.; Schlögl, R.; Hofmann, S. In Situ Characterization of Alloy Catalysts for Low-Temperature 
Graphene Growth. Nano Lett. 2011, 11 (10), 4154-4160.

https://doi.org/10.1021/n1202036y.

(38) Srivastava, A.; Galande, C.; Ci, L.; Song, L.; Rai, C.; Jariwala, D.; Kelly, K. F.; Ajayan, P. M. Novel Liquid Precursor-Based Facile Synthesis of Large-Area Continuous, Single, and Few-Layer Graphene Films. Chem. Mater. 2010, 22 (11), 3457-3461. https://doi.org/10.1021/cm101027c.

(39) Qi, J.; Zhang, L.; Cao, J.; Zheng, W.; Wang, X.; Feng, J. Synthesis of Graphene on a Ni Film by Radio-Frequency Plasma-Enhanced Chemical Vapor Deposition. Chin. Sci. Bull. 2012, 57 (23), 3040-3044. https://doi.org/10.1007/s11434-012-5120-4.

(40) Martins Ferreira, E. H.; Moutinho, M. V. O.; Stavale, F.; Lucchese, M. M.; Capaz, R. B.; Achete, C. A.; Jorio, A. Evolution of the Raman Spectra from Single-, Few-, and Many-Layer Graphene with Increasing Disorder. Phys. Rev. B 2010, 82 (12), 125429. https://doi.org/10.1103/PhysRevB.82.125429.

(41) Ferrari, A. C.; Basko, D. M. Raman Spectroscopy as a Versatile Tool for Studying the Properties of Graphene. Nat. Nanotechnol. 2013, 8 (4), 235-246. https://doi.org/10.1038/nnano.2013.46.

(42) Malard, L. M.; Pimenta, M. A.; Dresselhaus, G.; Dresselhaus, M. S. Raman Spectroscopy in Graphene. Phys. Rep. 2009, 473 (5), 51-87. https://doi.org/10.1016/j.physrep.2009.02.003.

(43) Graf, D.; Molitor, F.; Ensslin, K.; Stampfer, C.; Jungen, A.; Hierold, C.; Wirtz, L. Spatially Resolved Raman Spectroscopy of Single- and Few-Layer Graphene. Nano Lett. 2007, 7 (2), 238-242. https://doi.org/10.1021/n1061702a.

(44) Cançado, L. G.; Jorio, A.; Ferreira, E. H. M.; Stavale, F.; Achete, C. A.; Capaz, R. B.; Moutinho, M. V. O.; Lombardo, A.; Kulmala, T. S.; Ferrari, A. C. Quantifying Defects in Graphene via Raman Spectroscopy at Different Excitation Energies. Nano Lett. 2011, 11 (8), 3190-3196. https://doi.org/10.1021/nl201432g.

(45) Eckmann, A.; Felten, A.; Mishchenko, A.; Britnell, L.; Krupke, R.; Novoselov, K. S.; Casiraghi, C. Probing the Nature of Defects in Graphene by Raman Spectroscopy. Nano Lett. 2012, 12 (8), 3925-3930. https://doi.org/10.1021/nl300901a.

(46) Rocha, A. R.; Martins, T. B.; Fazzio, A.; da Silva, A. J. R. Disorder-Based Graphene Spintronics. Nanotechnology 2010, 21 (34), 345202. https://doi.org/10.1088/0957-4484/21/34/345202.

(47) Li, X.; Magnuson, C. W.; Venugopal, A.; Tromp, R. M.; Hannon, J. B.; Vogel, E. M.; Colombo, L.; Ruoff, R. S. Large-Area Graphene Single Crystals Grown by Low-Pressure Chemical Vapor Deposition of Methane on Copper. J. Am. Chem. Soc. 2011, 133 (9), 2816-2819. https://doi.org/10.1021/ja109793s.

(48) Yan, Z.; Lin, J.; Peng, Z.; Sun, Z.; Zhu, Y.; Li, L.; Xiang, C.; Samuel, E. L.; Kittrell, C.; Tour, J. M. Toward the Synthesis of Wafer-Scale Single-Crystal Graphene on Copper Foils. ACS Nano 2012, 6 (10), 9110-9117. https://doi.org/10.1021/nn303352k.

(49) Shi, B.-Y.; Cao, Q.-J.; Wang, Q.; Han, X.; Wu, H.-F.; Chu, L.-Q.; Fang, Z.-B.; Huang, H.; Tang, J.-X.; Dou, W.-D. Asymmetric Growth of Tetragon-Shaped Single-Crystalline Graphene Flakes on Copper Foil by Annealing Treatment under 
Oxygen-Free Conditions. J. Phys. Chem. C 2019, 123 (4), 2642-2650.

https://doi.org/10.1021/acs.jpcc.8b11897.

(50) Naghdi, S.; Rhee, K. Y.; Park, S. J. A Catalytic, Catalyst-Free, and Roll-to-Roll Production of Graphene via Chemical Vapor Deposition: Low Temperature Growth. Carbon 2018, 127, 1-12. https://doi.org/10.1016/j.carbon.2017.10.065.

(51) Kun, X.; Chen, X.; Jun, D.; Yanxu, Z.; Weiling, G.; Mingming, M.; Lei, Z.; Jie, S. Graphene Transparent Electrodes Grown by Rapid Chemical Vapor Deposition with Ultrathin Indium Tin Oxide Contact Layers for GaN Light Emitting Diodes. Appl. Phys. Lett. 2013, 102 (16), 162102. https://doi.org/10.1063/1.4802798.

(52) Yang, W.; He, C.; Zhang, L.; Wang, Y.; Shi, Z.; Cheng, M.; Xie, G.; Wang, D.; Yang, R.; Shi, D.; Zhang, G. Growth, Characterization, and Properties of Nanographene. Small 2012, 8 (9), 1429-1435. https://doi.org/10.1002/smll.201101827.

(53) Chan, S.-H.; Chen, S.-H.; Lin, W.-T.; Li, M.-C.; Lin, Y.-C.; Kuo, C.-C. LowTemperature Synthesis of Graphene on Cu Using Plasma-Assisted Thermal Chemical Vapor Deposition. Nanoscale Res. Lett. 2013, 8 (1), 285. https://doi.org/10.1186/1556-276X-8-285.

(54) Zeller, P.; Henß, A.-K.; Weinl, M.; Diehl, L.; Keefer, D.; Lippmann, J.; Schulz, A.; Kraus, J.; Schreck, M.; Wintterlin, J. Detachment of CVD-Grown Graphene from Single Crystalline Ni Films by a Pure Gas Phase Reaction. Surf. Sci. 2016, 653, 143-152. https://doi.org/10.1016/j.susc.2016.06.014.

(55) Lee, C.-S.; Shin, K. W.; Song, H.-J.; Park, H.; Cho, Y.; Im, D.-H.; Lee, H.; Lee, J.H.; Won, J. Y.; Chung, J. G.; Kim, C.; Byun, K.-E.; Lee, E.-K.; Kim, Y.; Ko, W.; Lim, H. J.; Park, S.; Shin, H.-J. Fabrication of Metal/Graphene Hybrid Interconnects by Direct Graphene Growth and Their Integration Properties. Adv. Electron. Mater. 2018, 4 (6), 1700624. https://doi.org/10.1002/aelm.201700624.

(56) Cui, S.; Cui, C.; Xie, J.; Liu, S.; Shi, J. Carbon Fibers Coated with Graphene Reinforced TiAl Alloy Composite with High Strength and Toughness. Sci. Rep. 2018, 8 (1), 2364. https://doi.org/10.1038/s41598-018-20799-y.

(57) Woo, Y. S.; Seo, D. H.; Yeon, D.-H.; Heo, J.; Chung, H.-J.; Benayad, A.; Chung, J.G.; Han, H.; Lee, H.-S.; Seo, S.; Choi, J.-Y. Low Temperature Growth of Complete Monolayer Graphene Films on Ni-Doped Copper and Gold Catalysts by a SelfLimiting Surface Reaction. Carbon 2013, 64, 315-323. https://doi.org/10.1016/j.carbon.2013.07.081.

(58) Seah, C.-M.; Chai, S.-P.; Mohamed, A. R. Mechanisms of Graphene Growth by Chemical Vapour Deposition on Transition Metals. Carbon 2014, 70, 1-21. https://doi.org/10.1016/j.carbon.2013.12.073.

(59) Cabrero-Vilatela, A.; Weatherup, R. S.; Braeuninger-Weimer, P.; Caneva, S.; Hofmann, S. Towards a General Growth Model for Graphene CVD on Transition Metal Catalysts. Nanoscale 2016, 8 (4), 2149-2158. https://doi.org/10.1039/C5NR06873H.

(60) Mehedi, H.-A.; Baudrillart, B.; Alloyeau, D.; Mouhoub, O.; Ricolleau, C.; Pham, V. D.; Chacon, C.; Gicquel, A.; Lagoute, J.; Farhat, S. Synthesis of Graphene by Cobalt-Catalyzed Decomposition of Methane in Plasma-Enhanced CVD: 
Optimization of Experimental Parameters with Taguchi Method. J. Appl. Phys. 2016, 120 (6), 065304. https://doi.org/10.1063/1.4960692.

(61) Lavin-Lopez, M. P.; Fernandez-Diaz, M.; Sanchez-Silva, L.; Valverde, J. L.; Romero, A. Improving the Growth of Monolayer CVD-Graphene over Polycrystalline Iron Sheets. New J. Chem. 2017, 41 (12), 5066-5074. https://doi.org/10.1039/C7NJ00281E.

(62) Weatherup, R. S.; Dlubak, B.; Hofmann, S. Kinetic Control of Catalytic CVD for High-Quality Graphene at Low Temperatures. ACS Nano 2012, 6 (11), 9996-10003. https://doi.org/10.1021/nn303674g.

(63) Wassei, J. K.; Mecklenburg, M.; Torres, J. A.; Fowler, J. D.; Regan, B. C.; Kaner, R. B.; Weiller, B. H. Chemical Vapor Deposition of Graphene on Copper from Methane, Ethane and Propane: Evidence for Bilayer Selectivity. Small 2012, 8 (9), 1415-1422. https://doi.org/10.1002/smll.201102276.

(64) Sun, X.; Lin, L.; Sun, L.; Zhang, J.; Rui, D.; Li, J.; Wang, M.; Tan, C.; Kang, N.; Wei, D.; Xu, H. Q.; Peng, H.; Liu, Z. Low-Temperature and Rapid Growth of Large Single-Crystalline Graphene with Ethane. Small 2018, 14 (3), 1702916. https://doi.org/10.1002/smll.201702916.

(65) Guermoune, A.; Chari, T.; Popescu, F.; Sabri, S. S.; Guillemette, J.; Skulason, H. S.; Szkopek, T.; Siaj, M. Chemical Vapor Deposition Synthesis of Graphene on Copper with Methanol, Ethanol, and Propanol Precursors. Carbon 2011, 49 (13), 42044210. https://doi.org/10.1016/j.carbon.2011.05.054.

(66) Li, Z.; Wu, P.; Wang, C.; Fan, X.; Zhang, W.; Zhai, X.; Zeng, C.; Li, Z.; Yang, J.; Hou, J. Low-Temperature Growth of Graphene by Chemical Vapor Deposition Using Solid and Liquid Carbon Sources. ACS Nano 2011, 5 (4), 3385-3390. https://doi.org/10.1021/nn200854p.

(67) Jang, J.; Son, M.; Chung, S.; Kim, K.; Cho, C.; Lee, B. H.; Ham, M.-H. LowTemperature-Grown Continuous Graphene Films from Benzene by Chemical Vapor Deposition at Ambient Pressure. Sci. Rep. 2015, 5, 17955. https://doi.org/10.1038/srep17955.

(68) Zhirnov, V. V.; Cavin, R. K.; Hutchby, J. A.; Bourianoff, G. I. Limits to Binary Logic Switch Scaling - a Gedanken Model. Proc. IEEE 2003, 91 (11), 1934-1939. https://doi.org/10.1109/JPROC.2003.818324.

(69) Skotnicki, T.; Hutchby, J. A.; Wong, and H.-P.; Boeuf, F. The End of CMOS Scaling: Toward the Introduction of New Materials and Structural Changes to Improve MOSFET Performance. IEEE Circuits Devices Mag. 2005, 21 (1), 16-26. https://doi.org/10.1109/MCD.2005.1388765.

(70) Nikonov, D. E.; Young, I. A. Benchmarking of Beyond-CMOS Exploratory Devices for Logic Integrated Circuits. IEEE J. Explor. Solid-State Comput. Devices Circuits 2015, 1, 3-11. https://doi.org/10.1109/JXCDC.2015.2418033.

(71) Wolf, S. A.; Awschalom, D. D.; Buhrman, R. A.; Daughton, J. M.; Molnár, S. von; Roukes, M. L.; Chtchelkanova, A. Y.; Treger, D. M. Spintronics: A Spin-Based Electronics Vision for the Future. Science 2001, 294 (5546), 1488-1495. https://doi.org/10.1126/science.1065389. 
(72) Kang, W.; Zhao, W.; Wang, Z.; Klein, J.; Zhang, Y.; Chabi, D.; Zhang, Y.; Ravelosona, D.; Chappert, C. An Overview of Spin-Based Integrated Circuits. In 2014 19th Asia and South Pacific Design Automation Conference (ASP-DAC); 2014; pp 676-683. https://doi.org/10.1109/ASPDAC.2014.6742969.

(73) Ralph, D. C.; Stiles, M. D. Spin Transfer Torques. J. Magn. Magn. Mater. 2008, 320 (7), 1190-1216. https://doi.org/10.1016/j.jmmm.2007.12.019.

(74) Sinova, J.; Žutić, I. New Moves of the Spintronics Tango. Nat. Mater. 2012, 11, 368-371. https://doi.org/10.1038/nmat3304.

(75) Yao, X.; Harms, J.; Lyle, A.; Ebrahimi, F.; Zhang, Y.; Wang, J. Magnetic Tunnel Junction-Based Spintronic Logic Units Operated by Spin Transfer Torque. IEEE Trans. Nanotechnol. 2012, 11 (1), 120-126. https://doi.org/10.1109/TNANO.2011.2158848.

(76) Lin, C.-C.; Penumatcha, A. V.; Gao, Y.; Diep, V. Q.; Appenzeller, J.; Chen, Z. Spin Transfer Torque in a Graphene Lateral Spin Valve Assisted by an External Magnetic Field. Nano Lett. 2013, 13 (11), 5177-5181. https://doi.org/10.1021/nl402547m.

(77) Balandin, A. A.; Ghosh, S.; Bao, W.; Calizo, I.; Teweldebrhan, D.; Miao, F.; Lau, C. N. Superior Thermal Conductivity of Single-Layer Graphene. Nano Lett. 2008, 8 (3), 902-907. https://doi.org/10.1021/n10731872.

(78) Geim, A. K.; Novoselov, K. S. The Rise of Graphene. Nat. Mater. 2007, 6 (3), 183191. https://doi.org/10.1038/nmat1849.

(79) Dutta, S.; Pati, S. K. Novel Properties of Graphene Nanoribbons: A Review. J. Mater. Chem. 2010, 20 (38), 8207-8223. https://doi.org/10.1039/C0JM00261E.

(80) Gao, Y.; Hao, P. Mechanical Properties of Monolayer Graphene under Tensile and Compressive Loading. Phys. E Low-Dimens. Syst. Nanostructures 2009, 41 (8), 1561-1566. https://doi.org/10.1016/j.physe.2009.04.033.

(81) Grigorenko, A. N.; Polini, M.; Novoselov, K. S. Graphene Plasmonics. Nat. Photonics 2012, 6 (11), 749-758. https://doi.org/10.1038/nphoton.2012.262.

(82) Park, C.-H.; Yang, L.; Son, Y.-W.; Cohen, M. L.; Louie, S. G. Anisotropic Behaviours of Massless Dirac Fermions in Graphene under Periodic Potentials. Nat. Phys. 2008, 4 (3), 213-217. https://doi.org/10.1038/nphys890.

(83) Pesin, D.; MacDonald, A. H. Spintronics and Pseudospintronics in Graphene and Topological Insulators. Nat. Mater. 2012, 11 (5), 409-416. https://doi.org/10.1038/nmat3305.

(84) Kamalakar, M. V.; Groenveld, C.; Dankert, A.; Dash, S. P. Long Distance Spin Communication in Chemical Vapour Deposited Graphene. Nat. Commun. 2015, 6, 6766. https://doi.org/10.1038/ncomms7766.

(85) Zhang, Y.; Tan, Y.-W.; Stormer, H. L.; Kim, P. Experimental Observation of the Quantum Hall Effect and Berry's Phase in Graphene. Nature 2005, 438 (7065), 201-204. https://doi.org/10.1038/nature04235.

(86) Józsa, C.; Maassen, T.; Popinciuc, M.; Zomer, P. J.; Veligura, A.; Jonkman, H. T.; van Wees, B. J. Linear Scaling between Momentum and Spin Scattering in Graphene. Phys. Rev. B 2009, 80 (24), 241403. https://doi.org/10.1103/PhysRevB.80.241403. 
(87) Drögeler, M.; Franzen, C.; Volmer, F.; Pohlmann, T.; Banszerus, L.; Wolter, M.; Watanabe, K.; Taniguchi, T.; Stampfer, C.; Beschoten, B. Spin Lifetimes Exceeding 12 Ns in Graphene Nonlocal Spin Valve Devices. Nano Lett. 2016, 16 (6), 35333539. https://doi.org/10.1021/acs.nanolett.6b00497.

(88) Kane, C. L.; Mele, E. J. Quantum Spin Hall Effect in Graphene. Phys. Rev. Lett. 2005, 95 (22), 226801. https://doi.org/10.1103/PhysRevLett.95.226801.

(89) Huertas-Hernando, D.; Guinea, F.; Brataas, A. Spin-Orbit-Mediated Spin Relaxation in Graphene. Phys. Rev. Lett. 2009, 103 (14). https://doi.org/10.1103/PhysRevLett.103.146801.

(90) Idzuchi, H.; Fukuma, Y.; Otani, Y. Spin Transport in Non-Magnetic NanoStructures Induced by Non-Local Spin Injection. Phys. E Low-Dimens. Syst. Nanostructures 2015, 68, 239-263. https://doi.org/10.1016/j.physe.2014.11.023.

(91) Ji, Y.; Hoffmann, A.; Jiang, J. S.; Pearson, J. E.; Bader, S. D. Non-Local Spin Injection in Lateral Spin Valves. J. Phys. Appl. Phys. 2007, 40 (5), 1280-1284. https://doi.org/10.1088/0022-3727/40/5/S13.

(92) Guimarães, M. H. D.; Veligura, A.; Zomer, P. J.; Maassen, T.; Vera-Marun, I. J.; Tombros, N.; van Wees, B. J. Spin Transport in High-Quality Suspended Graphene Devices. Nano Lett. 2012, 12 (7), 3512-3517. https://doi.org/10.1021/nl301050a.

(93) Patra, A. K.; Singh, S.; Barin, B.; Lee, Y.; Ahn, J.-H.; del Barco, E.; Mucciolo, E. R.; Özyilmaz, B. Dynamic Spin Injection into Chemical Vapor Deposited Graphene. Appl. Phys. Lett. 2012, 101 (16), 162407. https://doi.org/10.1063/1.4761932.

(94) Yang, T.-Y.; Balakrishnan, J.; Volmer, F.; Avsar, A.; Jaiswal, M.; Samm, J.; Ali, S. R.; Pachoud, A.; Zeng, M.; Popinciuc, M.; Güntherodt, G.; Beschoten, B.; Özyilmaz, B. Observation of Long Spin Relaxation Times in Bilayer Graphene at Room Temperature. 2010. https://doi.org/10.1103/PhysRevLett.107.047206.

(95) Goto, H.; Kanda, A.; Sato, T.; Tanaka, S.; Ootuka, Y.; Odaka, S.; Miyazaki, H.; Tsukagoshi, K.; Aoyagi, Y. Gate Control of Spin Transport in Multilayer Graphene. Appl. Phys. Lett. 2008, 92 (21), 212110. https://doi.org/10.1063/1.2937836.

(96) Tombros, N.; Jozsa, C.; Popinciuc, M.; Jonkman, H. T.; van Wees, B. J. Electronic Spin Transport and Spin Precession in Single Graphene Layers at Room Temperature. Nature 2007, 448 (7153), 571-574. https://doi.org/10.1038/nature06037.

(97) Raes, B.; Scheerder, J. E.; Costache, M. V.; Bonell, F.; Sierra, J. F.; Cuppens, J.; Van de Vondel, J.; Valenzuela, S. O. Determination of the Spin-Lifetime Anisotropy in Graphene Using Oblique Spin Precession. Nat. Commun. 2016, 7, 11444. https://doi.org/10.1038/ncomms 11444.

(98) Józsa, C.; Popinciuc, M.; Tombros, N.; Jonkman, H. T.; van Wees, B. J. Electronic Spin Drift in Graphene Field-Effect Transistors. Phys. Rev. Lett. 2008, 100 (23), 236603. https://doi.org/10.1103/PhysRevLett.100.236603.

(99) Han, W.; Pi, K.; McCreary, K. M.; Li, Y.; Wong, J. J. I.; Swartz, A. G.; Kawakami, R. K. Tunneling Spin Injection into Single Layer Graphene. Phys. Rev. Lett. 2010, 105 (16), 167202. https://doi.org/10.1103/PhysRevLett.105.167202. 
(100) Kochan, D.; Gmitra, M.; Fabian, J. Spin Relaxation Mechanism in Graphene: Resonant Scattering by Magnetic Impurities. Phys. Rev. Lett. 2014, 112 (11), 116602. https://doi.org/10.1103/PhysRevLett.112.116602.

(101) Tuan, D. V.; Ortmann, F.; Soriano, D.; Valenzuela, S. O.; Roche, S. PseudospinDriven Spin Relaxation Mechanism in Graphene. Nat. Phys. 2014, 10 (11), 857863. https://doi.org/10.1038/nphys3083.

(102) Schmidt, G.; Ferrand, D.; Molenkamp, L. W.; Filip, A. T.; van Wees, B. J. Fundamental Obstacle for Electrical Spin Injection from a Ferromagnetic Metal into a Diffusive Semiconductor. Phys. Rev. B 2000, 62 (8), R4790-R4793. https://doi.org/10.1103/PhysRevB.62.R4790.

(103) Dlubak, B.; Martin, M.-B.; Deranlot, C.; Bouzehouane, K.; Fusil, S.; Mattana, R.; Petroff, F.; Anane, A.; Seneor, P.; Fert, A. Homogeneous Pinhole Free 1 Nm Al2O3 Tunnel Barriers on Graphene. Appl. Phys. Lett. 2012, 101 (20), 203104. https://doi.org/10.1063/1.4765348.

(104) Volmer, F.; Drögeler, M.; Pohlmann, T.; Güntherodt, G.; Stampfer, C.; Beschoten, B. Contact-Induced Charge Contributions to Non-Local Spin Transport Measurements in Co/MgO/Graphene Devices. 2D Mater. 2015, 2 (2), 024001. https://doi.org/10.1088/2053-1583/2/2/024001.

(105) Berger, A. J.; Page, M. R.; Wen, H.; McCreary, K. M.; Bhallamudi, V. P.; Kawakami, R. K.; Chris Hammel, P. Correlating Spin Transport and Electrode Magnetization in a Graphene Spin Valve: Simultaneous Magnetic Microscopy and Non-Local Measurements. Appl. Phys. Lett. 2015, 107 (14), 142406. https://doi.org/10.1063/1.4932673.

(106) Popinciuc, M.; Józsa, C.; Zomer, P. J.; Tombros, N.; Veligura, A.; Jonkman, H. T.; van Wees, B. J. Electronic Spin Transport in Graphene Field-Effect Transistors. Phys. Rev. B 2009, 80 (21), 214427. https://doi.org/10.1103/PhysRevB.80.214427.

(107) Guimarães, M. H. D.; Zomer, P. J.; Ingla-Aynés, J.; Brant, J. C.; Tombros, N.; van Wees, B. J. Controlling Spin Relaxation in Hexagonal BN-Encapsulated Graphene with a Transverse Electric Field. Phys. Rev. Lett. 2014, 113 (8), 086602. https://doi.org/10.1103/PhysRevLett.113.086602.

(108) Boross, P.; Dóra, B.; Kiss, A.; Simon, F. A Unified Theory of Spin-Relaxation Due to Spin-Orbit Coupling in Metals and Semiconductors. Sci. Rep. 2013, 3, 3233. https://doi.org/10.1038/srep03233.

(109) Józsa, C.; Popinciuc, M.; Tombros, N.; Jonkman, H. T.; van Wees, B. J. Controlling the Efficiency of Spin Injection into Graphene by Carrier Drift. 2008. https://doi.org/10.1103/PhysRevB.79.081402.

(110) Ingla-Aynés, J.; Meijerink, R. J.; Wees, B. J. van. Eighty-Eight Percent Directional Guiding of Spin Currents with $90 \mathrm{Mm}$ Relaxation Length in Bilayer Graphene Using Carrier Drift. Nano Lett. 2016, 16 (8), 4825-4830. https://doi.org/10.1021/acs.nanolett.6b01004.

(111) Zietz, O.; Olson, S.; Coyne, B.; Liu, Y.; Jiao, J. Characterization and Manipulation of Carbon Precursor Species during Plasma Enhanced Chemical Vapor Deposition of Graphene. Nanomaterials 2020, 10 (11), 2235. https://doi.org/10.3390/nano10112235. 
(112) Guzmán, F.; Ruiz, M.; Valderrama, E.; Favre, M.; Bhuyan, H.; Wynham, E. S.; Chuaqui, H. Spectroscopic Characterization Of RF Hydrocarbon Plasmas For DLC Coatings. J. Phys. Conf. Ser. 2014, 511, 012017. https://doi.org/10.1088/17426596/511/1/012017.

(113) Baby, A.; Mahony, C. M. O.; Maguire, P. D. Acetylene-Argon Plasmas Measured at a Biased Substrate Electrode for Diamond-like Carbon Deposition: I. Mass Spectrometry. Plasma Sources Sci. Technol. 2011, 20 (1), 015003. https://doi.org/10.1088/0963-0252/20/1/015003.

(114) Denysenko, I. B.; Xu, S.; Long, J. D.; Rutkevych, P. P.; Azarenkov, N. A.; Ostrikov, K. Inductively Coupled Ar/CH4/H2 Plasmas for Low-Temperature Deposition of Ordered Carbon Nanostructures. J. Appl. Phys. 2004, 95 (5), 2713 2724. https://doi.org/10.1063/1.1642762.

(115) Ferrari, A. C.; Meyer, J. C.; Scardaci, V.; Casiraghi, C.; Lazzeri, M.; Mauri, F.; Piscanec, S.; Jiang, D.; Novoselov, K. S.; Roth, S.; Geim, A. K. Raman Spectrum of Graphene and Graphene Layers. Phys. Rev. Lett. 2006, 97 (18), 187401. https://doi.org/10.1103/PhysRevLett.97.187401.

(116) Ferrari, A. C. Raman Spectroscopy of Graphene and Graphite: Disorder, Electron-Phonon Coupling, Doping and Nonadiabatic Effects. Solid State Commun. 2007, 143 (1), 47-57. https://doi.org/10.1016/j.ssc.2007.03.052.

(117) Eckmann, A.; Felten, A.; Verzhbitskiy, I.; Davey, R.; Casiraghi, C. Raman Study on Defective Graphene: Effect of the Excitation Energy, Type, and Amount of Defects. Phys. Rev. B 2013, 88 (3), 035426. https://doi.org/10.1103/PhysRevB.88.035426.

(118) Increased Sample Yield and Achievable Imaging Resolutions Through Thin Film Transfer Technique | Microscopy and Microanalysis | Cambridge Core https://wwwcambridge-org.proxy.lib.pdx.edu/core/journals/microscopy-andmicroanalysis/article/increased-sample-yield-and-achievable-imaging-resolutionsthrough-thin-film-transfer-technique/910895C32DCF58290291E06AFAE9A9A1 (accessed Dec 8, 2020).

(119) Zhan, H.; Jiang, B.; Zietz, O.; Olson, S.; Jiao, J. Simulation to FabricationUnderstanding the Effect of NiAuCu Alloy Catalysts for Controlled Growth of Graphene at Reduced Temperature. Mater. Res. Express 2019, 7 (1), 015603. https://doi.org/10.1088/2053-1591/ab5bc3.

(120) Huang, M.; Ruoff, R. S. Growth of Single-Layer and Multilayer Graphene on $\mathrm{Cu} / \mathrm{Ni}$ Alloy Substrates. Acc. Chem. Res. 2020, 53 (4), 800-811. https://doi.org/10.1021/acs.accounts.9b00643.

(121) Tracy, J.; Zietz, O.; Olson, S.; Jiao, J. Plasma-Enhanced Chemical Vapor Deposition of Acetylene on Codeposited Bimetal Catalysts Increasing Graphene Sheet Continuity Under Low-Temperature Growth Conditions. Nanoscale Res. Lett. 2019, 14 (1), 335. https://doi.org/10.1186/s11671-019-3156-y.

(122) Coyne, B.; Zietz, O.; Olson, S.; Jiao, J. Graphene Layer Control Enabled by Nickel-Copper Alloy Thin Film Catalyst. Microsc. Microanal. 2018, 24 (S1), 16241625. https://doi.org/10.1017/S1431927618008607. 
(123) Wang, X.; Yuan, Q.; Li, J.; Ding, F. The Transition Metal Surface Dependent Methane Decomposition in Graphene Chemical Vapor Deposition Growth. Nanoscale 2017, 9 (32), 11584-11589. https://doi.org/10.1039/C7NR02743E.

(124) Segregation Growth of Graphene on $\mathrm{Cu}-\mathrm{Ni}$ Alloy for Precise Layer Control| The Journal of Physical Chemistry C https://pubs-acsorg.proxy.lib.pdx.edu/doi/abs/10.1021/jp202933u (accessed Dec 8, 2020).

(125) Zou, Z.; Carnevali, V.; Patera, L. L.; Jugovac, M.; Cepek, C.; Peressi, M.; Comelli, G.; Africh, C. Operando Atomic-Scale Study of Graphene CVD Growth at Steps of Polycrystalline Nickel. Carbon 2020, 161, 528-534.

https://doi.org/10.1016/j.carbon.2020.01.039.

(126) Chen, S.; Gao, J.; Srinivasan, B. M.; Zhang, G.; Sorkin, V.; Hariharaputran, R.; Zhang, Y.-W. An All-Atom Kinetic Monte Carlo Model for Chemical Vapor Deposition Growth of Graphene on $\mathrm{Cu}(1 \mathrm{~h}$ hspace $0.167 \mathrm{em} 1 \mathrm{\backslash hspace} 0.167 \mathrm{em} 1)$ Substrate. J. Phys. Condens. Matter 2020, 32 (15), 155401. https://doi.org/10.1088/1361-648X/ab62bf.

(127) Petrov, S.; Rafailov, P. M.; Marinova, V.; Lin, S.-H.; Lai, Y.-C.; Yu, P.; Chi, G.C.; Dimitrov, D. Z.; Karashanova, D.; Gospodinov, M. Chemical Vapor Deposition Growth of Bilayer Graphene via Altering Gas Flux Geometry. Thin Solid Films 2019, 690, 137521. https://doi.org/10.1016/j.tsf.2019.137521.

(128) Trimby, P. W. Orientation Mapping of Nanostructured Materials Using Transmission Kikuchi Diffraction in the Scanning Electron Microscope. Ultramicroscopy 2012, 120, 16-24. https://doi.org/10.1016/j.ultramic.2012.06.004.

(129) Avsar, A.; Ochoa, H.; Guinea, F.; Özyilmaz, B.; van Wees, B. J.; Vera-Marun, I. J. Colloquium: Spintronics in Graphene and Other Two-Dimensional Materials. Rev. Mod. Phys. 2020, 92 (2), 021003. https://doi.org/10.1103/RevModPhys.92.021003.

(130) Karpiak, B.; Cummings, A. W.; Zollner, K.; Vila, M.; Khokhriakov, D.; Hoque, A. M.; Dankert, A.; Svedlindh, P.; Fabian, J.; Roche, S.; Dash, S. P. Magnetic Proximity in a van Der Waals Heterostructure of Magnetic Insulator and Graphene. 2D Mater. 2019, 7 (1), 015026. https://doi.org/10.1088/2053-1583/ab5915.

(131) Zollner, K.; Gmitra, M.; Fabian, J. Heterostructures of Graphene and HBN: Electronic, Spin-Orbit, and Spin Relaxation Properties from First Principles. Phys. Rev. B 2019, 99 (12), 125151. https://doi.org/10.1103/PhysRevB.99.125151.

(132) Hsu, A.; Wang, H.; Kim, K. K.; Kong, J.; Palacios, T. Impact of Graphene Interface Quality on Contact Resistance and RF Device Performance. IEEE Electron Device Lett. 2011, 32 (8), 1008-1010. https://doi.org/10.1109/LED.2011.2155024.

(133) Liu, W.; Wei, J.; Sun, X.; Yu, H. A Study on Graphene-Metal Contact. Crystals 2013, 3 (1), 257-274. https://doi.org/10.3390/cryst3010257.

(134) Panda, J.; Ramu, M.; Karis, O.; Sarkar, T.; Kamalakar, M. V. Ultimate Spin Currents in Commercial Chemical Vapor Deposited Graphene. ACS Nano 2020, 14 (10), 12771-12780. https://doi.org/10.1021/acsnano.0c03376.

(135) Piquemal-Banci, M.; Galceran, R.; Dubois, S. M.-M.; Zatko, V.; Galbiati, M.; Godel, F.; Martin, M.-B.; Weatherup, R. S.; Petroff, F.; Fert, A.; Charlier, J.-C.; 
Robertson, J.; Hofmann, S.; Dlubak, B.; Seneor, P. Spin Filtering by Proximity Effects at Hybridized Interfaces in Spin-Valves with 2D Graphene Barriers. Nat. Commun. 2020, 11 (1), 5670. https://doi.org/10.1038/s41467-020-19420-6.

(136) Friedberg, R.; Paul, D. I. New Theory of Coercive Force of Ferromagnetic Materials. Phys. Rev. Lett. 1975, 34 (19), 1234-1237. https://doi.org/10.1103/PhysRevLett.34.1234.

(137) Lee, S.; Woo, J.; Lee, D.; Cha, E.; Park, J.; Moon, K.; Song, J.; Koo, Y.; Hwang, H. Tunnel Barrier Engineering of Titanium Oxide for High Non-Linearity of Selector-Less Resistive Random Access Memory. Appl. Phys. Lett. 2014, 104 (5), 052108. https://doi.org/10.1063/1.4864471.

(138) Zhao, B.; Khokhriakov, D.; Karpiak, B.; Hoque, A. M.; Xu, L.; Shen, L.; Feng, Y. P.; Xu, X.; Jiang, Y.; Dash, S. P. Electrically Controlled Spin-Switch and Evolution of Hanle Spin Precession in Graphene. 2D Mater. 2019, 6 (3), 035042. https://doi.org/10.1088/2053-1583/ab1d83.

(139) Lampert, L. High-Quality Chemical Vapor Deposition Graphene-Based Spin Transport Channels; 2000. https://doi.org/10.15760/etd.3308.

(140) Cherian, H. R.; Birge, N.; Pollanen, J.; Ahn, E. C. Fabrication of GrapheneInserted Tunneling Device (GiTD) for Emerging Spin Devices. ECS Trans. 2020, 98 (3), 3. https://doi.org/10.1149/09803.0003ecst.

(141) Ahn, E. C. 2D Materials for Spintronic Devices. Npj 2D Mater. Appl. 2020, 4 (1), 1-14. https://doi.org/10.1038/s41699-020-0152-0.

(142) Liu, Y.; Zeng, C.; Ding, J.; Zhong, J.; Gao, Y.; Kuang, X.; Yu, J.; Cao, L.; He, J.; Liu, Z. Effect of the Low-Resistance Tunnel Barriers Induced Inhomogeneous Spin Current Distribution in Graphene Crossed Configuration Lateral Spin Valve. AIP Adv. 2019, 9 (11), 115005. https://doi.org/10.1063/1.5088200.

(143) Khokhriakov, D.; Karpiak, B.; Hoque, A. Md.; Dash, S. P. Two-Dimensional Spintronic Circuit Architectures on Large Scale Graphene. Carbon 2020, 161, 892899. https://doi.org/10.1016/j.carbon.2020.01.103.

(144) Khokhriakov, D.; Hoque, A. M.; Karpiak, B.; Dash, S. P. Gate-Tunable SpinGalvanic Effect in Graphene-Topological Insulator van Der Waals Heterostructures at Room Temperature. Nat. Commun. 2020, 11 (1), 3657. https://doi.org/10.1038/s41467-020-17481-1.

(145) Dlubak, B.; Martin, M.-B.; Deranlot, C.; Servet, B.; Xavier, S.; Mattana, R.; Sprinkle, M.; Berger, C.; De Heer, W. A.; Petroff, F.; Anane, A.; Seneor, P.; Fert, A. Highly Efficient Spin Transport in Epitaxial Graphene on SiC. Nat. Phys. 2012, 8 (7), 557-561. https://doi.org/10.1038/nphys2331.

(146) Bandaru, P. R.; Daraio, C.; Jin, S.; Rao, A. M. Novel Electrical Switching Behaviour and Logic in Carbon Nanotube Y-Junctions. Nat. Mater. 2005, 4 (9), 663-666. https://doi.org/10.1038/nmat1450.

(147) Ganguly, S.; Basu, S. Spin Hall Conductance in Y-Shaped Junction Devices. J. Phys. Conf. Ser. 2017, 807 (8), 082003. https://doi.org/10.1088/17426596/807/8/082003. 